\title{
21. FORMATION MICROSCANNER LOGGING RESPONSES TO LITHOLOGY IN GUYOT CARBONATE PLATFORMS AND THEIR IMPLICATIONS: SITES 865 AND $866^{1}$
}

\author{
Patricia Cooper, ${ }^{2}$ Hubert M. Arnaud, ${ }^{3}$ and Peter G. Flood ${ }^{4}$
}

\begin{abstract}
Formation MicroScanner (FMS) images from Sites 865 and 866, Allison and Resolution guyots, respectively, were integrated with conventional logging data and core descriptions to provide detailed stratigraphic columns for those sites. First, large-scale (in hundreds of meters) lithologic units were determined from the conventional logs and compared to core descriptions; these units typically contain several major carbonate facies. Second, a "type" facies log response was established for each carbonate facies by correlating the best FMS images with the conventional log response and core information for the same depth. Finally, the remaining portions of the FMS images were interpreted using the "type" facies log responses as standards of comparison. It was immediately apparent that core recovery at both sites was highly preferential (e.g., within the alternating packstone-wackestone intervals, only small pebbles of well-cemented wackestone were recovered). This study indicated that packstone was the dominant lithology $(51 \%)$ at Hole $865 \mathrm{~A}$ and that grainstone $(25 \%)$ and packstone $(20 \%)$ were the dominant lithologies at Hole $866 \mathrm{~A}$ (excluding dolomite).

The sedimentary record, as determined from core-log integration, not only confirmed shipboard conclusions regarding the gross vertical trajectories of Allison and Resolution guyots, but provided details of facies changes that resulted from small-scale fluctuations in sea level. Deposition of the Albian section of Resolution Guyot overlaps in time with deposition of sediments at Allison Guyot. This study supports Strasser's conclusion (this volume) that a hiatus probably exists near the Albian/Aptian boundary at Resolution Guyot. Correlations of bed thicknesses and logging signatures between the two holes indicates that much of the Albian section may have been removed from Allison Guyot as it emerged above sea level.
\end{abstract}

\section{INTRODUCTION}

The central and northwestern Pacific Ocean seafloor is festooned with platforms and chains or groupings of seamounts, many of which are Cretaceous guyots, with their summits at depths of about $1500 \mathrm{~m}$ (Menard, 1964; Matthews et al., 1974; Winterer and Metzler, 1984; McNutt et al., 1990). Many of the volcanic edifices of the guyots are capped by shallow-water carbonate sediments. The sediments, which are almost always deposited at or near sea level, record the vertical trajectory of these guyots relative to sea level, caused by subsidence or uplift as well as fluctuations in eustatic sea level. The main scientific theme of the Leg 143 drilling program was the origin and evolution of Cretaceous guyots and their relationship to sea level. Thick deposits of carbonate platform sediments were sampled at Sites 865 and 866 , located in the lagoons of two drowned carbonate platforms in the MidPacific Mountains, Allison and Resolution, respectively (Fig. 1).

The formation of thick carbonate deposits requires a dynamic equilibrium between carbonate deposition and subsidence. The added dimension of time leads to the development of stratigraphic sequences that reflect changes in the depositional style. Variables affecting the style of deposition include changes in sea level, sedimentation rates, type of facies at the platform margin, and tectonic activity. A continuous vertical record of the stratigraphic sequence is necessary to determine the history of the relative influences of these variables on deposition at a particular site. For that reason, the stratigraphic record is usually the primary objective of scientific drilling, but is the most dif-

\footnotetext{
${ }^{1}$ Winterer, E.L., Sager, W.W., Firth, J.V., and Sinton, J.M. (Eds.), 1995. Proc. ODP Sci. Results, 143: College Station, TX (Ocean Drilling Program).

${ }^{2}$ Department of Geology and Geophysics, University of Hawaii, 2525 Correa Rd., Honolulu, HI 96822, U.S.A.

${ }^{3}$ Institut Dolomieu, Université de Grenoble, 15 rue Maurice Gignoux, 38031 Grenoble Cedex, France.

${ }^{4}$ Department of Geology and Geophysics, University of New England, Armidale, N.S.W. 2351, Australia.
}

ficult to obtain when using rotary $(\mathrm{RCB})$ drilling in reefal carbonates. Indeed, mean core recovery at Sites 865 and 866 was less than $16 \%$ and only $1 \%$ to $2 \%$ in the shallow-water limestones. Thus, logs are particularly important at those sites to address the primary objectives of the drilling (Shipboard Scientific Party, 1993). Downhole logs provide the essential addition of a continuous time-series measurement of the physical properties of the borehole wall. The purpose of this study is to integrate the conventional logging data, FMS images, and core information to provide detailed stratigraphic information that can constrain the vertical trajectories of the guyots, allowing one to correlate directly with other guyots drilled during Legs 143 and 144.

\section{DESCRIPTION AND INTERPRETATION OF FMS LOGS}

\section{Logging Program at Sites 865 and 866}

Ocean Drilling Program (ODP) Leg 143 obtained an extensive suite of in-situ borehole logging measurements (Sager, Winterer, Firth, et al., 1993). Hole $865 \mathrm{~A}$ (Fig. 1), which penetrated $870.9 \mathrm{~m}$ of calcareous oozes, wackestones and packstones, and basalts at Allison Guyot, was the only hole logged at Site 865 ; good logs were obtained within the open borehole between 100.5 and 867.0 mbsf. Five tool strings were run, including the sonic-porosity-density-gamma, the resistivitygamma, the geochemical tool string, the Japanese downhole magnetometer, and the Formation MicroScanner (FMS). Descriptions of the tools and their data characteristics are found in Shipboard Scientific Party (1993). The same five tool strings were run in Hole 866A at Resolution Guyot (Fig. 1) to cover the interval 74.5 to $1679.4 \mathrm{mbsf}$ for the first run only. Subsequent runs concluded at shallower depths because of cave-ins above $1679.4 \mathrm{mbsf}$, and the FMS was not run above 253.0 mbsf because of the extremely large hole size (>17.2 in. or $43.7 \mathrm{~cm}$ ).

In both holes, cycle skipping and other noise resulting from inadequate centralization of the tool compromised the quality of the sonic logs; hence, these were not used extensively for this analysis. Because of its mechanical problems, no valid data were recorded by the geochemical tool at either hole. Large hole diameters over 20 - to $35-\mathrm{m}$ 


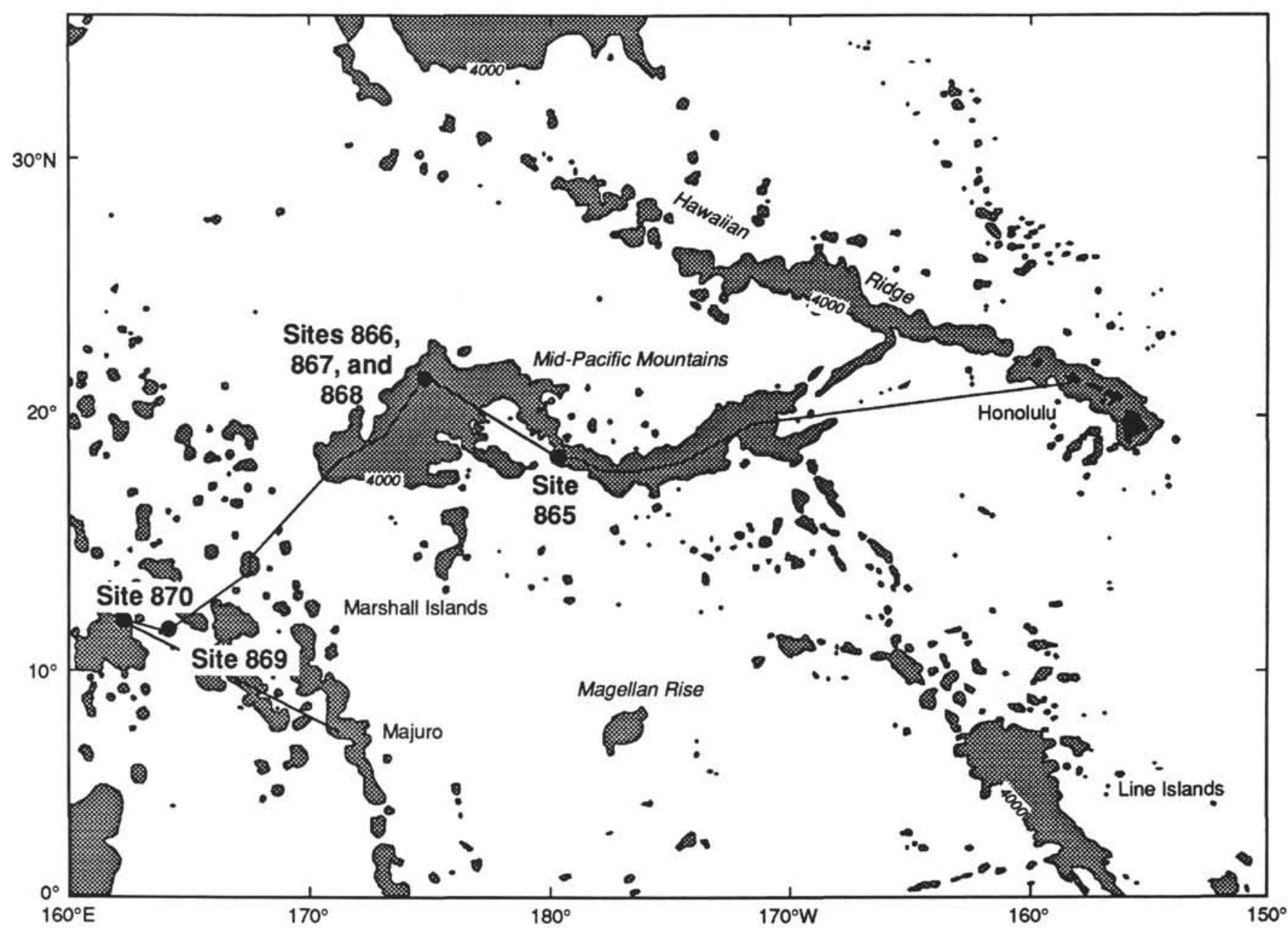

Figure 1. Location of Leg 143 drill sites and principal seamount chains, western central Pacific Ocean basin. Stippled areas are shallower than $4 \mathrm{~km}$. Solid line shows track of the JOIDES Resolution.

intervals account for questionable porosity/density data for large portions of both holes.

\section{FMS Resistivity Measurements}

The resistivity $\log$ is a combined measure of the resistivities of both the rock-forming minerals and the fluids contained in the pore spaces. The constitutive minerals of common sedimentary rocks are highly resistive; hence, water content and salinity are the two most important factors that control measured electrical resistivity. Other factors that can influence resistivity include the concentration of hydrous and metallic minerals, vesicularity, the geometry of interconnected pore spaces, and, to a lesser extent, temperature. Research has shown that, to a first-order approximation, resistivity is proportional to the inverse square root of the porosity (Archie, 1942).

In pure limestones, electrical current is conducted almost entirely by the brine contained in the pore space. High resistivities correspond to low porosities; low resistivities correspond to high porosities. Wet clays conduct electricity, and the magnitude of this conduction effect in clayey limestones will depend on the percentage of clay content and clay mineral composition (Archie, 1952).

The FMS produces high-resolution resistivity measurements of the borehole wall (Ekstrom et al., 1987) that are ideal for detailed sedimentological studies (e.g., Bourke et al., 1989; Adams et al., 1990; Luthi, 1990; Harker et al., 1990). The tool consists of 16 electrodes on each of four orthogonal pads that press against the borehole wall. Electrodes are spaced about $2.5 \mathrm{~mm}$ apart in two diagonally offset rows. The focussed current that flows from the electrodes is recorded as a series of measurements of the variations in resistivity with respect to depth. Shore-based processing converts these measurements into spatially oriented images of the borehole wall. With a sampling interval of $2.5 \mathrm{~mm}$, the vertical resolution is $2.5 \mathrm{~mm}$ (Serra, 1989). Coverage of the borehole wall for each pass of the tool varies inversely with hole diameter-about $40 \%$ of an 8.5 -in. hole, less for greater diameters. Perhaps the most important limitation of the tool is the restriction to hole diameters of less than $38.1 \mathrm{~cm}$ ( $15 \mathrm{in}$.). Some information may be gleaned from the image if one or two pads make good contact with the borehole wall, as is the case with highly elliptical holes, but often no useful information is contained in FMS images from washed out sections.

The resistivity values of the surfaces sampled by the pads are converted to gray scale and plotted as a set of vertical strips with an indication of pad orientation. This oriented image (Figs. 2-7) corresponds to an unrolled cylinder with a width that is equivalent to the diameter of the borehole. Horizontal and vertical scales are the same. In general, low resistivities (high conductivities) are shown as dark tones, whereas high resistivities (low conductivities) are shown as light tones. The image tone is a qualitative representation of electrical resistivity because, when processing the data, normalization procedures are applied to optimize the contrast on the images. Images are usually interpreted with the aid of conventional resistivity logs to quantify the variation of electrical resistivity within the formation. 


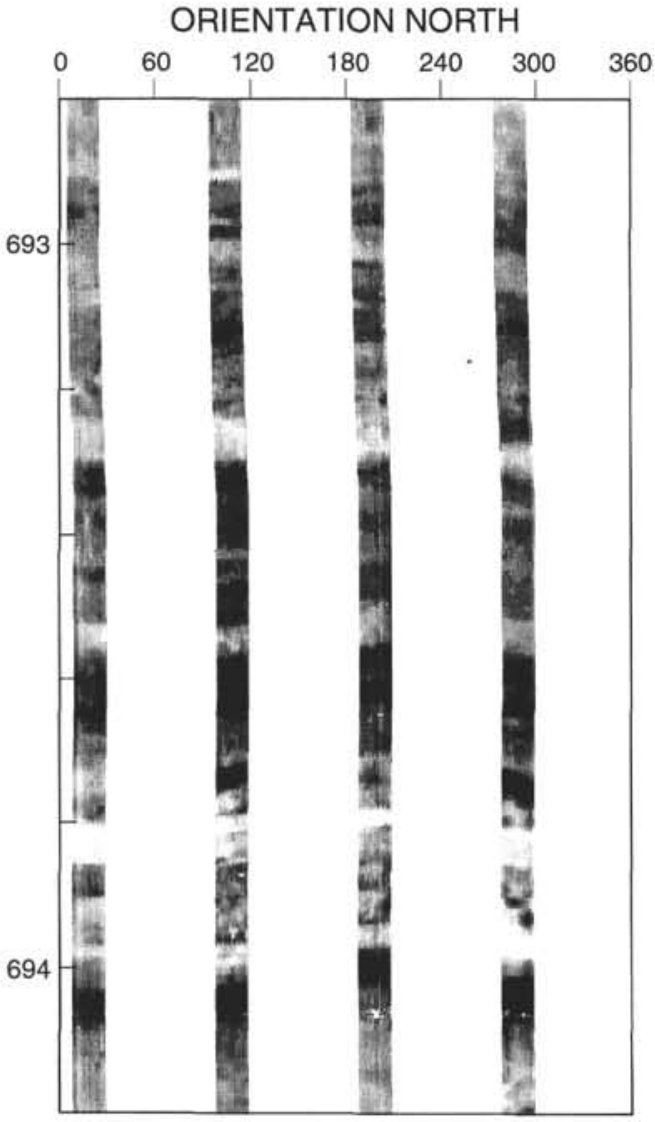

Figure 2. FMS image from 692.8 to $694.2 \mathrm{mbsf}$, Hole $866 \mathrm{~A}$, interpreted as grainstone texture showing intervals of greater (light) and lesser (dark) cementation. Dark spots are molds or vugs.

\section{Methodology for Integration of Conventional Logging Data, FMS Images, and Core Data}

Integration of the FMS, conventional logs, and cores proceeded in the following way: First, conventional resistivity, velocity, porosity, density, gamma, and caliper logs were plotted together for $100-\mathrm{m}$ intervals. This multiple-trace presentation facilitated determination of large-scale trends and patterns. The major sedimentary units were identified on the basis of baseline shifts or similar variability, mainly in the resistivity and natural gamma-ray logs (see Shipboard Scientific Party, 1993). These major sedimentary units then were identified in the FMS images.

To do this, the FMS logs were depth-matched to the conventional logs by correlating the gamma-ray logs between runs. A linear depth shift was applied to all logs to correct for differences in the stretch of the logging cable, using the gamma log from the first logging run as reference. These depths should be accurate to within $\pm 2 \mathrm{~m}$ of the drillers' depths. All logging depths (recorded as feet below rig floor) were then corrected to meters below seafloor by subtracting the depth from the rig floor to the seafloor. Short, repeat sections of the FMS for both holes were compared to differentiate between geologic reality and processing artifact.

Second, the identification of a "type" log facies for each recovered core facies was accomplished by intercomparison of FMS, conventional logs, and core descriptions. The FMS and conventional logs were displayed and inspected simultaneously to identify conventional $\log$ responses that corresponded to characteristic patterns observed on the FMS image. Long ( $>10 \mathrm{~m}$ ) FMS and conventional logging sections with reasonably uniform characteristics were then compared to descriptions of core material taken from the same depth range. Spe-

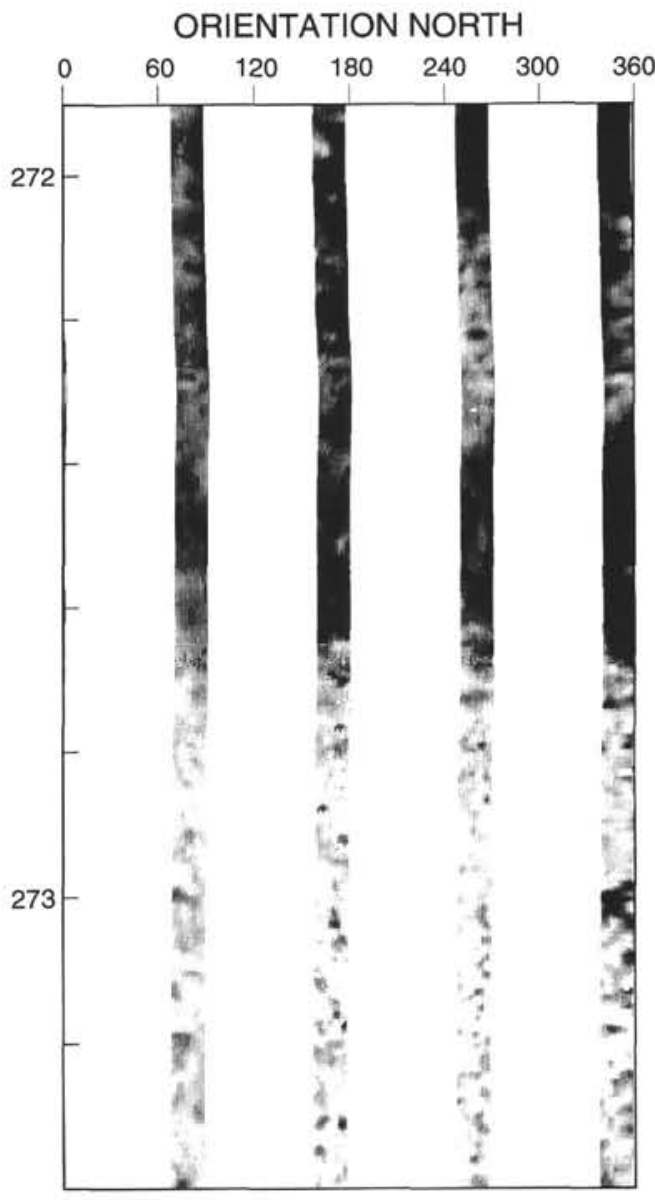

Figure 3. This FMS image from 271.9 to $273.4 \mathrm{mbsf}$, Hole 866A, interpreted as a packstone (top)-wackestone (bottom) texture, presents a mottled appearance with irregular white spots on an irregular dark gray background. Dark spots are molds or vugs.

cial care was exercised because core recovery was poor and obviously preferential; typically, within the alternating packstone-wackestone intervals, only small pebbles of the well-cemented wackestones were recovered. However, we reasoned that if the logging responses were similar over a given depth interval and only one lithology was recovered over that interval, then we could be certain that our interlog correlation as a "type" facies was accurate. The classification of shallowwater carbonate textures used here is the same as that used in Sager, Winterer, Firth, et al. (1993), namely, that of Dunham (1962). Fortunately, the thick sections of wackestone-packstones and grainstones provided multiple "type" facies that could be checked against each other. The remaining portions of the FMS logs were interpreted by comparison to the "type" facies logging responses.

The greatest source of error in the identification of facies in the FMS images was hole size. Hole diameters for more than two-thirds of Hole $865 \mathrm{~A}$ and one-third of Hole $866 \mathrm{~A}$ were larger than the maximum opening diameter $(38.1 \mathrm{~cm})$ of the FMS calipers. The FMS data were of poor quality for the depth intervals 102-145, 198-212, 248-296, 305-322, 373-440, 500-527, 565-638, 651-702, and 746791 in Hole 865A, and 200-262, 375-390, 455-661, 802-825, 976$993,1037-1054,1078-1106,1124-1152,1278-1292$, and $1317-1340$ in Hole 866A. Poor pad contact in these portions of the hole degraded the quality of the images. On some images two, sometimes only one, pads made good contact with the borehole wall; nonetheless, the images could still be interpreted based on comparisons to the type log facies. The conventional logs did not require contact with the borehole wall; corrections for the effects of variations in hole size were applied in post-cruise data processing. 


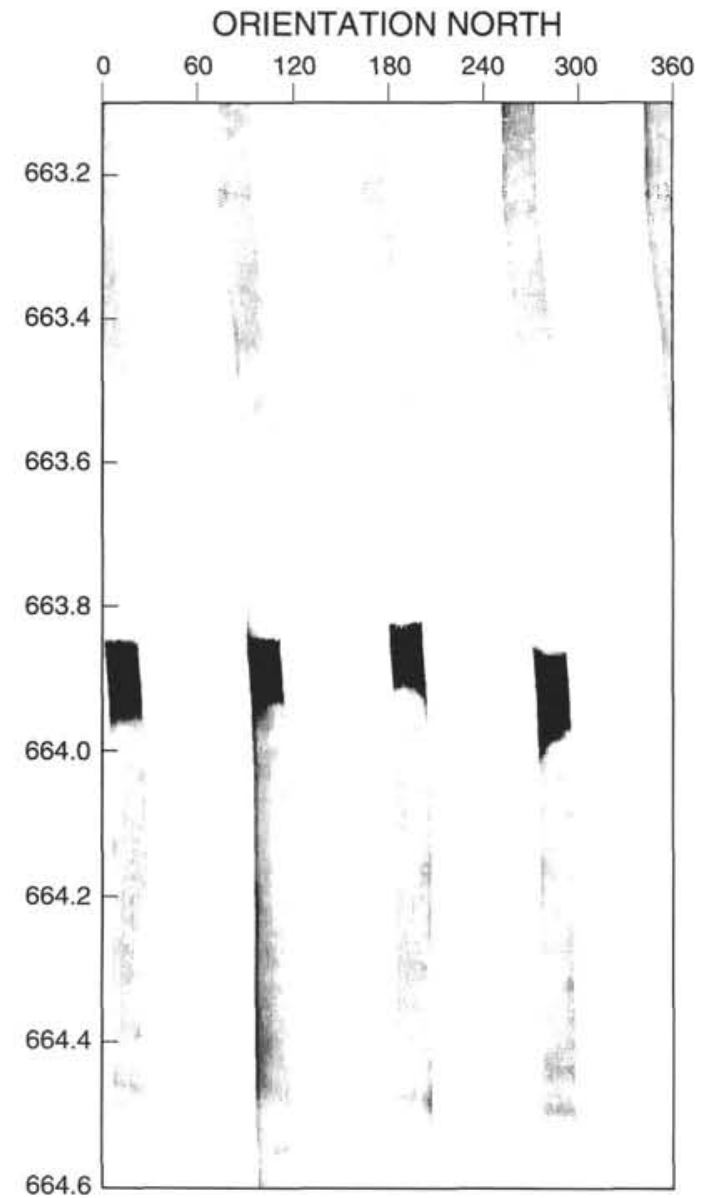

Figure 4. FMS image from 663.1 to $664.6 \mathrm{mbsf}$, Hole 866A. A mudstone presents a relatively homogeneous white to light gray image, depending on the porosity (the lower the porosity, the whiter the image). The black band above $664.0 \mathrm{mbsf}$ is a clay seam.

\section{FMS IMAGE INTERPRETATIONS}

Keeping in mind that the image tone is not directly proportional to the resistivity value, the dark tones of the FMS image usually correspond to materials that are conductive because of high intergranular porosity, fine-grained textures, or the presence of conductive minerals (e.g., clay and pyrite). The light tones correspond to materials that are resistive, either because of low intergranular porosity (e.g., mudstones) or coarse-grained texture (Serra, 1989). Identification of lithology was sometimes clear and straightforward, sometimes intuitive. For example, thick $(>1 \mathrm{~cm})$ organic- and clay-rich facies were easily identifiable in the FMS image as sharply defined black bands and in conventional logs, as a sharp, low-resistivity peak corresponding to a high-gamma peak. Thin layers or disseminated clay and organic-rich matter (as reported in the core descriptions) produced subtle logging responses that would have been difficult to interpret without calibration by the core material. Some minor lithologies, notably boundstone, were difficult to identify accurately in the FMS images because these produced no characteristic logging response aside from low density values, which are common to several carbonate textures. Combining the core descriptions with boundstone texture described by Serra (1989), we concluded that the boundstone texture was characterized by irregular, dark features (molds) in a gray background with white, somewhat vertical, interconnecting features that would result from the continuous, cemented framework, and with white spots or streaks (shell fragments).

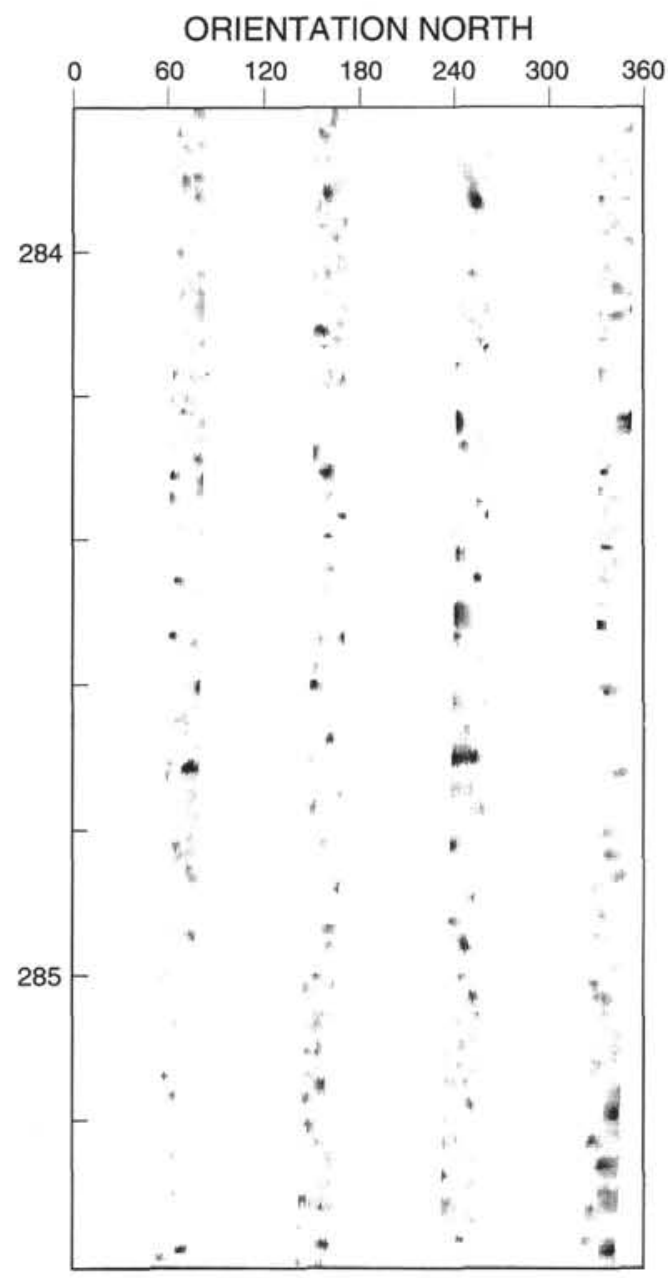

Figure 5. FMS image from 283.8 to $285.4 \mathrm{mbsf}$, Hole 866A. Images of mudstones with extreme moldic porosity closely resemble those of wackestone; the two textures can be distinguished only by their relative densities.

Grainstone texture was characterized by obvious layering (Fig. 2); individual layers having a constant gray tone corresponded to wellsorted intervals. Packstones and wackestones presented a mottled appearance as a result of their highly variable grain sizes and porosities, with irregular white spots on an irregular gray background, sometimes with dark spots (molds or vugs). Wackestones (Fig. 3, bottom portion) contain more lime mud than do packstones (Fig. 3, top portion) and therefore are more resistive. Mudstones produced a relatively homogeneous white to light gray tone depending on the porosity (Fig. 4). Images of mudstone having extensive moldic porosity (Fig. 5) closely resembled packstone-wackestone, but could be distinguished on the basis of relatively higher density in the conventional logs. Rudstone texture is characterized by irregular white spots (packstone-wackestone) isolated in a dark background and in the conventional logs by low density, resistivity, and sonic velocity. Dolomite often displays a texture similar to rudstone: leached and moldic porosities combine to present a dark background (Fig. 6), but much higher resistivity values in the conventional log. Partial dolomitization is indicated in the FMS image by dark areas that correspond to zones of higher porosity that may cross bedding planes (e.g., in the grainstones, Fig. 7).

Figure 8 provides a key to patterns that indicate carbonate textures in Figures 9 and 10. Figures 9 and 10 display the conventional logs most important for interpreting the FMS images, including resistivity, gamma-ray, density, and caliper, the core number and amount recovered, together with interpretation of the lithology. The pattern indicates 


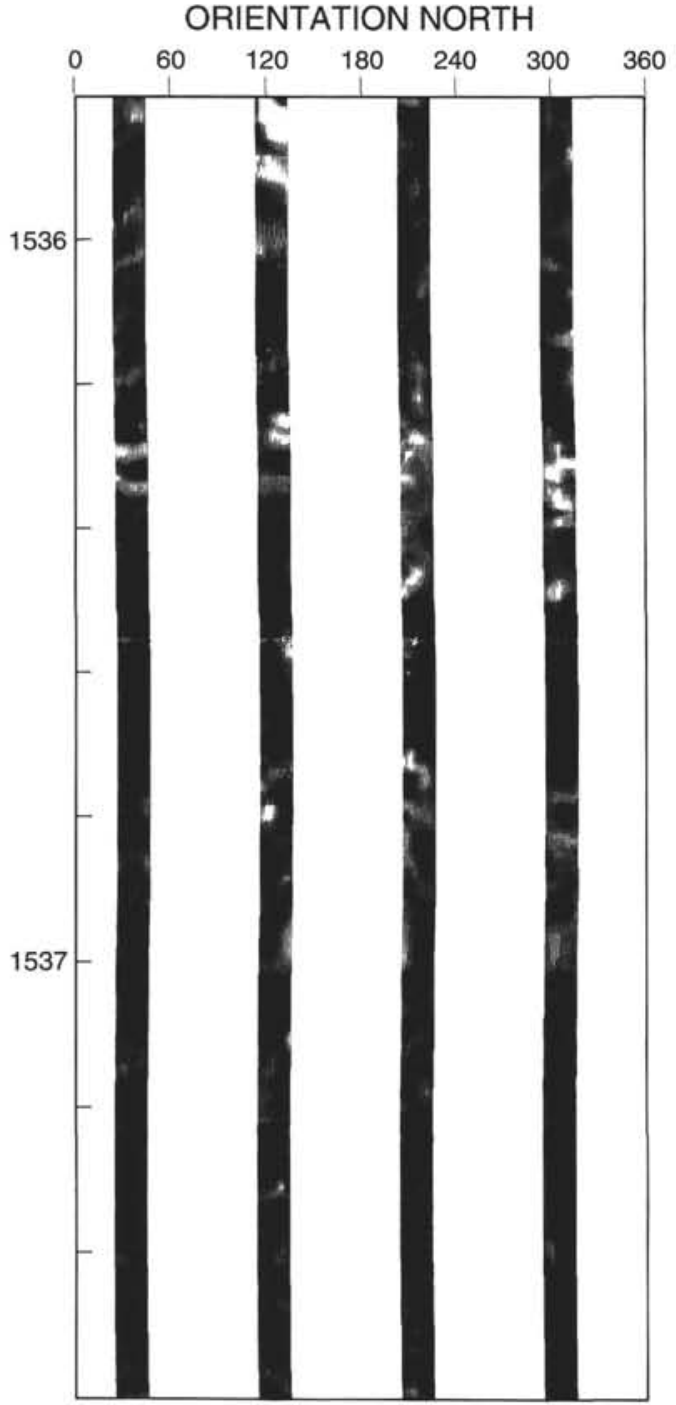

Figure 6. FMS image from 1535.8 to $1537.6 \mathrm{mbsf}$, Hole 866A. Dolomites typically display a rudstone texture (i.e., irregular white spots [dense, redeposited carbonate]) in an irregular, dark gray to black background (leached and moldic porosities).

the dominant lithology for an interval; in some cases, a split pattern is shown to indicate almost equal interbeds of another lithology. Split patterns also are used to indicate partial dolomitization or a dolomitized section having relict texture. Because of the limited range of black- and-white patterns and shadings available, more detailed descriptions of the facies are listed in Tables 1 (Hole 865A) and 2 (Hole $866 \mathrm{~A}$ ). Occasionally, the core material was matched to the FMS images on the basis of such distinctive characteristics as size and amount of moldic porosity, presence of stylolites or clay seams, among others. Core numbers are placed in the comment section of the bed or beds from which they may have originated. In many cases, especially in the thin packstone-wackestone interbeds, it was not possible to identify the place of origin of the core material with confidence.

\section{EFFECTS OF LITHOLOGY ON CORING}

Overall recovery in both holes was poor, averaging only about $2 \%$ in the shallow-water limestones, despite repeated efforts to improve recovery by changing the style of coring. Instead of smoothly cut core, hard, shelly wackestone was recovered as small chunks with

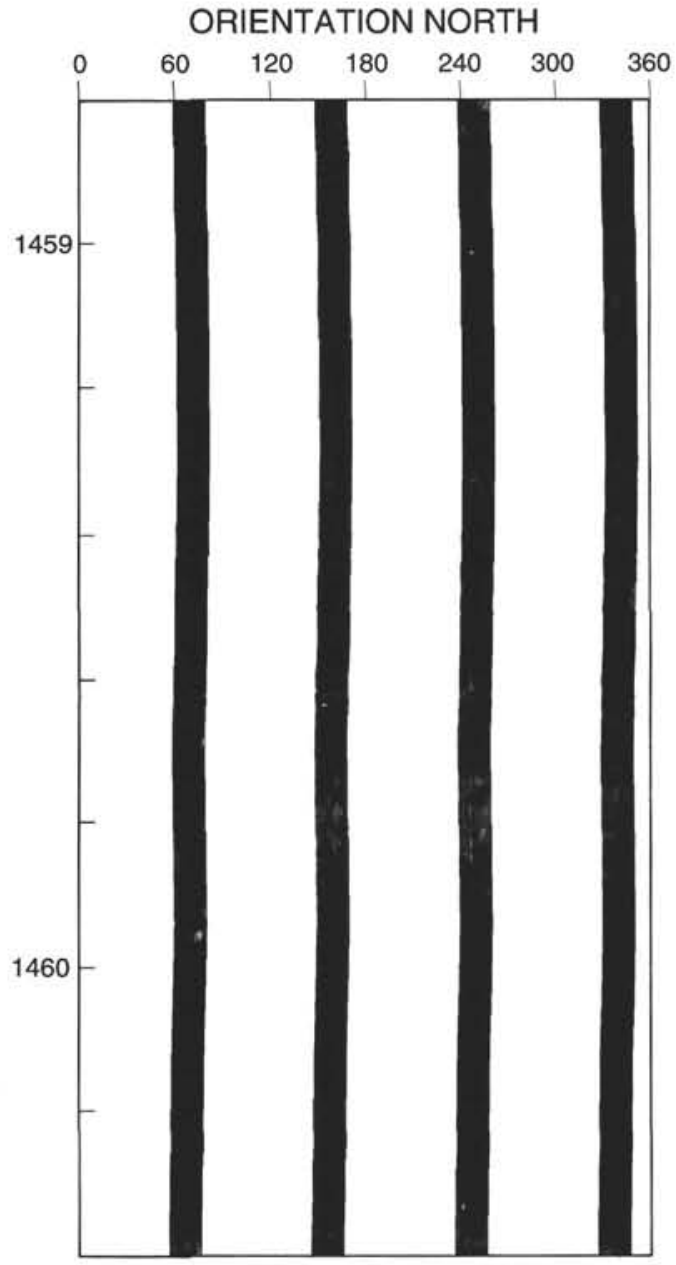

Figure 7. FMS image from 1458.8 to $1460.4 \mathrm{mbsf}$, Hole 866A. Dolomitization occurs preferentially within the most porous facies, the grainstones. In this example, some grainstone texture remains, although dark areas, corresponding to zones of higher porosity, cut across bedding planes.

typical dimensions of 1 to $4 \mathrm{~cm}$. These hard limestone layers constitute thin, scattered layers; the bulk of the sediment consists of limestones too porous or weakly cemented to core using the available tools. In intervals where variations in cementation were present, lower resistivity and density values directly corresponded to large hole diameters; soft, poorly indurated material was preferentially removed during the drilling process. Recovery was lowest in strata having intergranular and extreme moldic porosity (packstones and grainstones) and highest in clay-rich or dolomitized strata. For example, as clay content and dolomitization increased downhole at Site 866 from about $600 \mathrm{mbsf}$, recovery increased to about $50 \%$ in the lowest $50 \mathrm{~m}$ of the hole.

The FMS log reveals more well-cemented intervals than were recovered as core. Recovery in wackestone-packstone (hard-soft) intervals having thicknesses on the order of 1 to $2 \mathrm{~m}$ was particularly poor. In some cored intervals, it was possible to locate the recovered material at its source depth (Tables 1 and 2); a pattern of recovery is suggested such that when coring begins or ends in a well-lithified layer, some of that layer is usually recovered.

Table 3 summarizes the statistical distribution of lithologies interpreted from the core-log integration at Holes 865A and 866 A. All beds containing predominantly grainstone (e.g., grainstone-packstone, grainstone-rudstone) were grouped under grainstone; similarly, all beds containing predominantly rudstone (e.g., rudstone-packstone, 
Table 1. Description of beds at Hole 865A (Allison Guyot).

\begin{tabular}{|c|c|c|c|}
\hline $\begin{array}{c}\text { Bed } \\
\text { number }\end{array}$ & $\begin{array}{l}\text { Depth } \\
\text { range (mbsf) }\end{array}$ & Lithology & Description \\
\hline 1 & $135.0-140.0$ & Foraminifer-nannofossil ooze & Subtly mottled \\
\hline 2 & $140.0-143.31$ & Packstone & \\
\hline 3 & $143.31-143.76$ & Wackestone & Moldic porosity \\
\hline 4 & $143.76-146.13$ & Packstone & \\
\hline 5 & $146.13-147.25$ & Wackestone & Moldic porosity \\
\hline 6 & $147.25-149.93$ & Packstone-wackestone & $\begin{array}{l}\text { Moldic porosity; minor floatstone (148.2-148.4) } \\
\text { (Core 18R) }\end{array}$ \\
\hline 7 & $149.93-152.05$ & Wackestone-packstone & Moldic porosity \\
\hline 8 & $152.05-155.40$ & Packstone & Moldic porosity \\
\hline 9 & $155.40-156.59$ & Wackestone & Moldic porosity \\
\hline 10 & $156.59-157.39$ & Packstone & \\
\hline 11 & $157.39-158.27$ & Packstone-wackestone & \\
\hline 12 & $158.27-158.84$ & Packstone & Uniform grain size \\
\hline 13 & $158.84-159.24$ & Wackestone & \\
\hline 14 & $159.24-160.96$ & Packstone & $\begin{array}{l}\text { Minor moldic porosity; minor wackestone } \\
\text { intervals }\end{array}$ \\
\hline 15 & $160.96-167.70$ & Packstone-wackestone & $\begin{array}{l}\text { Moldic porosity concentrated at distinct horizons; } \\
\text { well-cemented intervals (mudstone?) }\end{array}$ \\
\hline 16 & $167.70-180.92$ & Packstone & $\begin{array}{l}\text { Highly porous and vugular; wackestone intervals } \\
(167.6-167.8,172.2-173.2) \text {; large vug }(170.7- \\
\text { 171.32); highly resistive intraclasts (calcite) } \\
\text { (Core 21R) }\end{array}$ \\
\hline 17 & $180.92-181.62$ & Wackestone-packstone & Interbedded; vugular \\
\hline 18 & $181.62-182.15$ & Packstone & \\
\hline 19 & $182.15-185.60$ & Wackestone-packstone & Variable cementation; moldic (gastropod) porosity \\
\hline 20 & $185.60-186.52$ & Mudstone-wackestone & Extreme moldic porosity \\
\hline 21 & $186.52-186.90$ & Mudstone-wackestone & Minor moldic porosity \\
\hline 22 & $186.90-197.07$ & Packstone & $\begin{array}{l}\text { Vugular and moldic porosity; large vug (190.36- } \\
190.64 \text { ) }\end{array}$ \\
\hline 23 & $197.07-197.59$ & Wackestone & Moldic porosity (Core 23R) \\
\hline 24 & $197.59-222.32$ & Packstone & $\begin{array}{l}\text { Moldic porosity; grossly vugular in places; minor } \\
\text { mudstone }\end{array}$ \\
\hline 25 & $222.32-224.10$ & Packstone-wackestone & Moldic porosity \\
\hline 26 & $224.10-223.13$ & Packstone & $\begin{array}{l}\text { Moldic porosity; grossly vugular in places; well- } \\
\text { cemented interval (227.9-228.1) }\end{array}$ \\
\hline 27 & $223.13-234.29$ & Packstone-wackestone & Moldic porosity \\
\hline 28 & $234.29-239.56$ & Packstone & Moldic porosity; grossly vugular in places \\
\hline 29 & $239.56-245.11$ & Wackestone & Moldic porosity (Core 28R) \\
\hline 30 & $245.11-267.95$ & Packstone & Moldic porosity; grossly vugular in places \\
\hline 31 & $267.95-271.60$ & Wackestone-mudstone & Well-indurated in places; porous \\
\hline 32 & $271.60-272.60$ & Mudstone-wackestone & Very minor moldic porosity \\
\hline 33 & $272.60-280.27$ & Packstone & Minor moldic porosity; well-indurated \\
\hline 34 & $280.27-282.80$ & Packstone-rudstone & \\
\hline 35 & $282.80-288.90$ & Wackestone-mudstone & Moldic porosity (Core $32 \mathrm{R}$ ) \\
\hline 36 & $288.90-290.73$ & Packstone & Moldic porosity \\
\hline 37 & $290.73-299.46$ & Packstone-wackestone & Moldic porosity \\
\hline 38 & $299.46-299.69$ & Packstone & \\
\hline 39 & $299.69-307.43$ & Packstone-grainstone & Minor moldic porosity (Core $34 \mathrm{R}$ ) \\
\hline 40 & $307.43-311.05$ & Wackestone-mudstone & Moldic porosity \\
\hline 41 & $311.05-317.72$ & Packstone & Moldic porosity; large vugs; very porous \\
\hline 42 & $317.72-333.80$ & Wackestone-mudstone & $\begin{array}{l}\text { Moldic porosity; interbedded, but bedding is } \\
\text { indistinct; minor grainstone (Cores } 36 \mathrm{R}, 37 \mathrm{R} \text { ) }\end{array}$ \\
\hline 43 & $333.80-336.97$ & Packstone-grainstone & Minor moldic porosity \\
\hline 44 & $336.97-338.25$ & Packstone & Very porous; few well-indurated intervals \\
\hline 45 & $338.25-340.58$ & Wackestone-mudstone & \\
\hline 46 & $340.58-341.20$ & Mudstone & $\begin{array}{l}\text { Moldic porosity; very large vugs }(3-4 \mathrm{~cm} \text { ) (Core } \\
\text { 38R) }\end{array}$ \\
\hline 47 & $341.20-344.31$ & Packstone & \\
\hline 48 & $344.31-344.40$ & Mudstone & \\
\hline 49 & $344.40-347.26$ & Packstone & Minor moldic porosity; extremely porous zones \\
\hline 50 & $347.26-348.00$ & Packstone-grainstone & Minor moldic porosity \\
\hline 51 & $348.00-349.00$ & Wackestone-packstone & $\begin{array}{l}\text { Finely interbedded with minor grainstone (Core } \\
39 \mathrm{R} \text { ) }\end{array}$ \\
\hline 52 & $349.00-355.73$ & Packstone & \\
\hline 53 & $355.73-357.60$ & Packstone-grainstone & \\
\hline 54 & $357.60-359.20$ & Packstone-wackestone & (Core 41R) \\
\hline 55 & $359.20-364.09$ & Packstone-grainstone & (Core 4IR) \\
\hline 56 & $364.09-370.00$ & Packstone-wackestone & Moldic porosity; extremely porous zones \\
\hline 57 & $370.00-405.98$ & Packstone & $\begin{array}{l}\text { Moldic porosity; burrows; well-indurated interval } \\
(396.08-397.12)\end{array}$ \\
\hline 58 & $405.98-409.15$ & Packstone & Very porous \\
\hline 59 & $409.15-410.95$ & Mudstone-wackestone & Mudstone intraclasts (Core 46R) \\
\hline 60 & $410.95-416.23$ & Packstone & Very porous; moldic porosity; burrowed \\
\hline 61 & $416.23-417.20$ & Mudstone-wackestone & Moldic porosity; burrowed \\
\hline 62 & $417.20-417.40$ & Mudstone & Minor moldic porosity \\
\hline 63 & $417.40-421.10$ & Packstone & Very porous \\
\hline 64 & $421.10-423.80$ & Wackestone-mudstone & $\begin{array}{l}\text { Some bedding visible; minor moldic porosity; } \\
\text { burrowed }\end{array}$ \\
\hline 65 & $423.80-425.80$ & Mudstone-wackestone & Moldic porosity \\
\hline 66 & $425.80-426.80$ & Packstone-wackestone & Minor moldic porosity \\
\hline 67 & $426.80-428.87$ & Packstone & Very porous \\
\hline 68 & $428.87-429.16$ & Mudstone & \\
\hline 69 & $429.16-430.25$ & Wackestone-mudstone & Stylolites \\
\hline 70 & $430.25-432.87$ & Wackestone-mudstone & Wacke. grading up to mud \\
\hline 71 & $432.87-434.20$ & Wackestone-mudstone & Wacke. grading up to mud; mudstone intraclast \\
\hline 72 & $434.20-436.15$ & Wackestone-mudstone & Wacke. grading up to wacke.-mud \\
\hline 73 & $436.15-438.20$ & Wackestone-mudstone & Wacke. grading up to mud-wacke. \\
\hline
\end{tabular}


Table 1 (continued).

\begin{tabular}{|c|c|c|c|}
\hline $\begin{array}{c}\text { Bed } \\
\text { number }\end{array}$ & $\begin{array}{l}\text { Depth } \\
\text { range (mbsf) }\end{array}$ & Lithology & Description \\
\hline 74 & $438.20-440.77$ & Wackestone-mudstone & $\begin{array}{l}\text { Very porous wacke. with moldic porosity grading } \\
\text { up to mudstone }\end{array}$ \\
\hline 75 & $440.77-441.42$ & Packstone & \\
\hline 76 & $441.42-445.24$ & Mudstone-wackestone & Minor moldic porosity; burrowed \\
\hline 77 & $445.24-446.48$ & Wackestone-mudstone & Moldic porosity; burrowed \\
\hline 78 & $446.48-447.97$ & Packstone & \\
\hline 79 & $447.97-448.42$ & Wackestone-mudstone & \\
\hline 80 & $448.42-450.40$ & Mudstone-wackestone & Moldic porosity (Core 50R) \\
\hline 81 & $450.40-452.29$ & Wackestone-mudstone & \\
\hline 82 & $452.29-454.02$ & Packstone & Very porous, (mudstone?) intraclasts \\
\hline 83 & $454.02-456.37$ & Wackestone-mudstone & Moldic porosity \\
\hline 84 & $456.37-457.63$ & Mudstone-wackestone & Minor moldic porosity; burrowed (Core 51R) \\
\hline 85 & $457.63-461.80$ & Wackestone-mudstone & Some bedding visible; burrowed \\
\hline 86 & $461.80-462-92$ & Mudstone-wackestone & Large patches with high intergranular porosity \\
\hline 87 & $462.92-465.40$ & Packstone & Minor mudstone interbeds \\
\hline 88 & $465.40-466.62$ & Wackestone-mudstone & Moldic porosity; burrowed \\
\hline 89 & $466.62-467.07$ & Mudstone-wackestone & \\
\hline 90 & $467.07-469.14$ & Wackestone-mudstone & Minor moldic porosity; burrowed \\
\hline 91 & $469.14-472.20$ & Mudstone-wackestone & Moldic porosity; burrowed \\
\hline 92 & $472.20-476.60$ & Packstone & Very porous, intraclasts \\
\hline 93 & $476.60-476.98$ & Packstone & \\
\hline 94 & $476.98-477.11$ & Grainstone & \\
\hline 95 & $477.11-478.71$ & Wackestone-mudstone & Wacke grading up to mudstone; burrowed \\
\hline 96 & $478.71-481.50$ & Wackestone-mudstone & Moldic porosity, burrowed (Core 53R) \\
\hline 97 & $481.50-483.30$ & Packstone & Burrowed \\
\hline 98 & $483.30-485.90$ & Packstone & $\begin{array}{l}\text { Irregular patches of mudstone; intraclasts; } \\
\text { burrowed; some bedding visible }\end{array}$ \\
\hline 99 & $485.90-486.79$ & Wackestone-mudstone & Minor moldic porosity \\
\hline 100 & $486.79-488.26$ & Packstone & \\
\hline 101 & $488.26-489.63$ & Wackestone-mudstone & \\
\hline 102 & $489.63-490.60$ & Mudstone-wackestone & Minor moldic porosity \\
\hline 103 & $490.60-491.62$ & Packstone & Sharp basal contact \\
\hline 104 & $491.62-493.64$ & Mudstone-wackestone & Moldic porosity \\
\hline 105 & $493.64-496.40$ & Wackestone-mudstone & Some bedding visible; moldic porosity \\
\hline 106 & $496.40-496.64$ & Mudstone & (Core 54R) \\
\hline 107 & $496.64-498.35$ & Packstone & Borrowed \\
\hline 108 & $498.35-501.91$ & Mudstone-wackestone & \\
\hline 109 & $501.91-504.08$ & Packstone & Burrowed \\
\hline 110 & $504.08-507.69$ & Wackestone-mudstone & Intraclasts \\
\hline 111 & $507.69-509.25$ & Mudstone-wackestone & \\
\hline $\begin{array}{l}112 \\
113\end{array}$ & $\begin{array}{l}509.25-509.97 \\
509.97-513.77\end{array}$ & $\begin{array}{l}\text { Wackestone-mudstone } \\
\text { Packstone }\end{array}$ & Very porous at base \\
\hline 114 & $513.77-516.64$ & Wackestone-mudstone & $\begin{array}{l}\text { Minor moldic porosity; some bedding visible at } \\
\text { top (Core 57R) }\end{array}$ \\
\hline 115 & $516.64-524.27$ & Packstone & $\begin{array}{l}\text { Highly variable intergranular porosity; moldic } \\
\text { porosity }\end{array}$ \\
\hline 116 & $524.27-527.00$ & Wackestone-mudstone & Some bedding visible in lower half \\
\hline 117 & $527.00-529.85$ & Packstone & \\
\hline 118 & $529.85-530.92$ & Wackestone-mudstone & \\
\hline 119 & $530.92-532.45$ & Packstone & \\
\hline 120 & $532.45-534.87$ & Wackestone-mudstone & Moldic porosity; burrowed (Core 58R) \\
\hline 121 & $534.87-537.00$ & Packstone & Mudstone interbeds at base; minor moldic porosity \\
\hline 122 & $537.00-541.38$ & Packstone & Moldic porosity; burrowed \\
\hline 123 & $541.38-544.19$ & Wackestone-mudstone & Moldic porosity; burrowed (Core 59R) \\
\hline 124 & $544.19-544.80$ & Mudstone & Moldic porosity \\
\hline 125 & $544.80-547.20$ & Wackestone-mudstone & \\
\hline 126 & $547.20-549.80$ & Packstone & Very porous \\
\hline 127 & $549.80-551.89$ & Wackestone-mudstone & Moldic porosity \\
\hline 128 & $551.89-553.00$ & Mudstone-wackestone & Minor moldic porosity (Core 60R) \\
\hline 129 & $553.00-557.26$ & Mudstone & Moldic porosity \\
\hline 130 & $557.26-561.00$ & Wackestone-mudstone & Moldic porosity; burrowed (Core 61R) \\
\hline 131 & $561.00-564.40$ & Packstone & Minor moldic porosity; bottom third is burrowed \\
\hline 132 & $564.40-566.00$ & Wackestone-mudstone & Moldic porosity \\
\hline 133 & $566.00-567.90$ & Mudstone-wackestone & Moldic porosity; burrowed \\
\hline 134 & $567.90-568.63$ & Wackestone-mudstone & Minor moldic porosity \\
\hline 135 & $568.63-571.00$ & Packstone & Moldic porosity; burrowed \\
\hline 136 & $571.00-573.00$ & Wackestone-mudstone & Moldic porosity; burrowed \\
\hline 137 & $573.00-578.35$ & Packstone & Moldic porosity; burrowed \\
\hline 138 & $578.35-579.91$ & Wackestone-mudstone & Moldic porosity; burrowed \\
\hline 139 & $579.91-581.20$ & Mudstone-wackestone & Moldic porosity; burrowed \\
\hline 140 & $581.20-587.00$ & Wackestone-mudstone & Moldic porosity; burrowed \\
\hline 141 & $587.00-587.50$ & Mudstone & Massive \\
\hline 142 & $587.50-588.78$ & Wackestone-mudstone & Moldic porosity; burrowed (Core 64R) \\
\hline 143 & $588.78-592.63$ & Packstone & Porous; moldic porosity; burrowed \\
\hline 144 & $592.63-593.25$ & Wackestone-mudstone & Minor moldic porosity \\
\hline 145 & $593.25-598.40$ & Packstone & Very porous \\
\hline 146 & $598.40-600.83$ & Wackestone-mudstone & Moldic porosity; burrowed; some bedding visible \\
\hline 147 & $600.83-603.17$ & Wackestone-mudstone & Moldic porosity; burrowed \\
\hline 148 & $603.17-603.63$ & Mudstone & Stratified \\
\hline 149 & $603.63-608.34$ & Packstone & $\begin{array}{l}\text { Moldic porosity; intraclasts (may be filled } \\
\text { burrows); burrowed }\end{array}$ \\
\hline 150 & $608.34-609.40$ & Mudstone & Extreme moldic porosity \\
\hline 151 & $609.40-610.12$ & Packstone & Moldic porosity \\
\hline 152 & $610.12-610.42$ & Mudstone & Moldic porosity \\
\hline 153 & $610.42-614.56$ & Wackestone-mudstone & Stratified; minor moldic porosity \\
\hline 154 & $614.56-616.36$ & Mudstone-wackestone & $\begin{array}{l}\text { Stratification partially obscured by burrows; minor } \\
\text { moldic porosity }\end{array}$ \\
\hline 155 & $616.36-619.23$ & Packstone & Moldic porosity; somewhat burrowed (Core 67R) \\
\hline
\end{tabular}


Table 1 (continued).

\begin{tabular}{|c|c|c|c|}
\hline $\begin{array}{c}\text { Bed } \\
\text { number }\end{array}$ & $\begin{array}{l}\text { Depth } \\
\text { range (mbsf) }\end{array}$ & Lithology & Description \\
\hline 156 & $619.23-622.30$ & Wackestone-mudstone & Moldic porosity; burrowed \\
\hline 157 & $622.30-622.59$ & Mudstone & Sharp basal contact \\
\hline 158 & $622.59-627.80$ & Packstone & Moldic porosity; burrowed \\
\hline 159 & $627.80-630.42$ & Mudstone-wackestone & Moldic porosity; burrowed \\
\hline 160 & $630.42-638.51$ & Packstone & $\begin{array}{l}\text { Very porous; moldic porosity; burrowed; } \\
\text { mudstone interbeds }(631.6,636.3)\end{array}$ \\
\hline 161 & $638.51-641.60$ & Wackestone-mudstone & $\begin{array}{l}\text { Well-cemented; moldic porosity; minor } \\
\text { bioturbation (Core 69R) }\end{array}$ \\
\hline 162 & $641.60-645.28$ & Packstone & Moldic porosity; burrowed \\
\hline 163 & $645.28-649.11$ & Wackestone-mudstone & $\begin{array}{l}\text { Well-cemented; moldic porosity; extensively } \\
\text { bioturbated (Core 70R) }\end{array}$ \\
\hline 164 & $649.11-650.72$ & Packstone & $\begin{array}{l}\text { Regular patches of packstone; minor moldic } \\
\text { porosity }\end{array}$ \\
\hline 165 & $650.72-653.40$ & Packstone & Moldic porosity; burrowed \\
\hline 166 & $653.40-655.56$ & Mudstone-wackestone & $\begin{array}{l}\text { Muddy wackestone with mud interbeds; moldic } \\
\text { porosity; minor bioturbation }\end{array}$ \\
\hline 167 & $655.56-660.76$ & Packstone & $\begin{array}{l}\text { Extensive bioturbation; moldic porosity; clay } \\
\text { seams (656.6) (Core 71R) }\end{array}$ \\
\hline 168 & $660.76-661.61$ & Packstone & Moldic porosity; burrowed \\
\hline 169 & $661.61-662.02$ & Mudstone & Finely interbedded clay seams \\
\hline 170 & $662.02-665.16$ & Packstone & Moldic porosity; burrowed \\
\hline 171 & $665.16-665.69$ & Wackestone-packstone & $\begin{array}{l}\text { Minor moldic porosity; stylolites; clay seams } \\
\text { (Core } 72 R \text { ) }\end{array}$ \\
\hline 172 & $665.69-667.23$ & Packstone & Moldic porosity: minor bioturbation \\
\hline 173 & $667.23-668.43$ & Wackestone-packstone & Interbedded; minor moldic porosity; burrowed \\
\hline 174 & $668.43-670.03$ & Packstone & Very porous; minor bioturbation \\
\hline 175 & $670.03-670.12$ & Wackestone & \\
\hline 176 & $670.12-672.20$ & Packstone & Minor moldic porosity; burrowed \\
\hline 177 & $672.20-672.70$ & Wackestone & \\
\hline 178 & $672.70-673.22$ & Packstone & \\
\hline 179 & $673.22-673.70$ & Wackestone & \\
\hline 180 & $673.70-675.20$ & Packstone & $\begin{array}{l}\text { Minor moldic porosity; burrowed; identical to bed } \\
176\end{array}$ \\
\hline 181 & $675.20-675.70$ & Mudstone & Finely interbedded clay seams \\
\hline 182 & $675.70-678.17$ & Mudstone-wackestone & $\begin{array}{l}\text { Minor moldic porosity; extensive bioturbation in } \\
\text { places }\end{array}$ \\
\hline 183 & $678.17-680.92$ & Packstone & Very porous; moldic porosity \\
\hline 184 & $680.92-681.30$ & Packstone-grainstone & Minor moldic porosity \\
\hline 185 & $681.30-683.72$ & Grainstone & \\
\hline 186 & $683.72-684.37$ & Packstone-grainstone & Minor moldic porosity \\
\hline 187 & $684.37-689.32$ & Packstone-wackestone & $\begin{array}{l}\text { Packstone with distinct wackestone intervals; } \\
\text { moldic porosity; individual burrows very } \\
\text { distinct (Core 74R) }\end{array}$ \\
\hline 188 & $689.32-689.77$ & Wackestone & \\
\hline 189 & $689.77-694.38$ & Packstone & Moldic porosity; burrowed \\
\hline 190 & $694.38-695.27$ & Wackestone & Minor bioturbation \\
\hline 191 & $695.27-696.36$ & Packstone & Upper half extensively burrowed; moldic porosity \\
\hline 192 & $696.36-696.77$ & Wackestone & \\
\hline 193 & $696.77-698.24$ & Packstone-wackestone & Burrowed \\
\hline 94 & $698.24-699.00$ & Mudstone & \\
\hline 195 & $699.00-699.38$ & Mudstone-wackestone & \\
\hline 196 & $699.38-702.20$ & Packstone & Minor bioturbation \\
\hline 197 & $702.20-702.56$ & Wackestone & \\
\hline 198 & $702.56-703.51$ & Packstone & Extensively burrowed \\
\hline 199 & $703.51-703.97$ & Wackestone & \\
\hline 200 & $703.97-706.73$ & Wackestone-packstone & Minor moldic porosity \\
\hline 201 & $706.73-709.05$ & Packstone & Burrowed \\
\hline 202 & $709.05-709.24$ & Grainstone-packstone & \\
\hline 203 & $709.24-711.17$ & Packstone-wackestone & Minor moldic porosity; burrowed; intraclasts \\
\hline 204 & $711.17-712.80$ & Wackestone & $\begin{array}{l}\text { Clay seam (712.45); minor moldic porosity (Core } \\
77 \mathrm{R})\end{array}$ \\
\hline 205 & $712.80-725.60$ & Packstone & $\begin{array}{l}\text { Mudstone interbeds }(716.6,720.85,722.0) \text {; minor } \\
\text { moldic porosity }\end{array}$ \\
\hline 206 & $725.60-727.35$ & Wackestone-mudstone & Moldic porosity; burrowed (Core 78R) \\
\hline 207 & $727.35-727.60$ & Mudstone & \\
\hline 208 & $727.60-727.68$ & Clay & \\
\hline 209 & $727.68-728.11$ & Marly limestone & \\
\hline 210 & $728.11-728.75$ & Mudstone & Minor moldic porosity; burrowed \\
\hline 211 & $728.75-732.67$ & Packstone-wackestone & Minor moldic porosity; burrowed (Core 79R) \\
\hline 212 & $732.67-735.86$ & Packstone & $\begin{array}{l}\text { Very fine-grained; minor wackestone at base } \\
\text { (Core 80R) }\end{array}$ \\
\hline 213 & $735.86-738.29$ & Mudstone & Finely interbedded clay seams \\
\hline 214 & $738.29-744.74$ & Wackestone-mudstone & $\begin{array}{l}\text { Moldic porosity; extensively burrowed; minor } \\
\text { packstone intervals }\end{array}$ \\
\hline 215 & $744.74-747.50$ & Packstone & Moldic porosity \\
\hline 216 & $747.50-748.55$ & Clay & Minor mudstone \\
\hline 217 & $748.55-750.36$ & Wackestone-mudstone & Moldic porosity: moderately burrowed \\
\hline 218 & $750.36-750.90$ & Mudstone-wackestone & Minor moldic porosity; extensively burrowed \\
\hline 219 & $750.90-751.22$ & Wackestone-mudstone & Burrowed \\
\hline 220 & $751.22-751.83$ & Mudstone & Clayey limestone-mud \\
\hline 221 & $751.83-754.52$ & Wackestone-mudstone & Minor moldic porosity; minor bioturbation \\
\hline 222 & $754.52-755.39$ & Wackestone & Clay seams \\
\hline 223 & $755.39-755.85$ & Packstone-wackestone & Minor moldic porosity \\
\hline 224 & $755.85-761.91$ & Packstone & $\begin{array}{l}\text { Moldic porosity; large burrows; clay seams (Core } \\
82 \mathrm{R} \text { ) }\end{array}$ \\
\hline 225 & $761.91-763.80$ & Mudstone-wackestone & Moldic porosity; burrowed \\
\hline
\end{tabular}


Table 1 (continued).

\begin{tabular}{|c|c|c|c|}
\hline $\begin{array}{c}\text { Bed } \\
\text { number }\end{array}$ & $\begin{array}{l}\text { Depth } \\
\text { range (mbsf) }\end{array}$ & Lithology & Description \\
\hline 226 & $763.80-763.90$ & Mudstone & Clay seams \\
\hline 227 & $763.90-764.23$ & Mudstone-wackestone & Burrowed \\
\hline 228 & $764.23-765.60$ & Wackestone-packstone & Moldic porosity; burrowed \\
\hline 229 & $765.60-769.00$ & Packstone & $\begin{array}{l}\text { Minor }(10-\mathrm{cm}) \text { packstone intervals; moldic } \\
\text { porosity; extensively burrowed }\end{array}$ \\
\hline 230 & $769.00-769.35$ & Wackestone & \\
\hline 231 & $769.35-770.02$ & Wackestone-packstone & Moldic porosity; burrowed \\
\hline 232 & $770.02-771.67$ & Packstone & Moldic porosity; intraclasts \\
\hline 233 & $771.67-772.20$ & Wackestone-packstone & Burrowed (Core 83R) \\
\hline 234 & $771.20-776.00$ & Packstone & Moldic porosity \\
\hline 235 & $776.00-776.94$ & Wackestone & Clay seams \\
\hline 236 & $776.94-778.30$ & Packstone & Minor moldic porosity; burrowed \\
\hline 237 & $778.30-779.38$ & Mudstone-wackestone & Clay seam at 779.32 ; minor moldic porosity \\
\hline 238 & $779.38-782.14$ & Wackestone-mudstone & Very fine-scale moldic porosity \\
\hline 239 & $782.14-782.23$ & Clay & \\
\hline 240 & $782.23-783.15$ & Mudstone-wackestone & Clay seam at 782.8 ; minor moldic porosity \\
\hline 241 & $783.15-793.23$ & Packstone & $\begin{array}{l}\text { Abundant intraclasts at base; vertical fluid escape } \\
\text { channels (or burrows) at base; upward fining }\end{array}$ \\
\hline 242 & $793.23-793.53$ & Laminated clayey limestone & (Core 85R) \\
\hline 243 & $793.53-796.12$ & Mudstone-wackestone & Extensively burrowed (Core 85R) \\
\hline 244 & $796.12-798.79$ & Wackestone-mudstone & Moldic porosity; extensively burrowed \\
\hline 245 & $798.79-799.13$ & Mudstone & \\
\hline 246 & $799.13-799.97$ & Packstone & $\begin{array}{l}\text { Intraclasts (mudstone), dark (pyrite)-filled } \\
\text { burrows }\end{array}$ \\
\hline 247 & $799.97-802.35$ & Marl & \\
\hline 248 & $802.35-803.03$ & Packstone & Clay seams; intraclasts \\
\hline 249 & $803.03-804.40$ & Marl & Intraclasts \\
\hline 250 & $804.40-806.86$ & Wackestone & $\begin{array}{l}\text { Clay seams; moldic porosity; extensively } \\
\text { burrowed }\end{array}$ \\
\hline 251 & $806.86-806.91$ & Clay & \\
\hline 252 & $806.91-809.20$ & Packstone & Clay seams; intraclasts, burrowed \\
\hline 253 & $809.20-811.96$ & Marl & $\begin{array}{l}\text { Clay seams; fragments of conductive material; } \\
\text { extensively burrowed (Core } 87 \mathrm{R} \text { ) }\end{array}$ \\
\hline 254 & $811.96-813.24$ & Marl & Minor wackestone; clay seams \\
\hline 255 & $813.24-816.34$ & Packstone-wackestone & Burrowed \\
\hline 256 & $816.34-819.80$ & Packstone & \\
\hline $\begin{array}{l}257 \\
258\end{array}$ & $819.80-820.70$ & Packstone-marl & \\
\hline $\begin{array}{l}258 \\
259\end{array}$ & $\begin{array}{l}820.70-823.55 \\
823.55-824.29\end{array}$ & & $\begin{array}{l}\text { Large burrows; angular fragments (Core } 88 \mathrm{R} \text { ) } \\
\text { Burrowed }\end{array}$ \\
\hline 260 & $824.29-825.20$ & $\begin{array}{l}\text { Packstone-wackestone } \\
\text { Packstone }\end{array}$ & $\begin{array}{l}\text { Burrowed } \\
\text { Large burrows }\end{array}$ \\
\hline 261 & $825.20-825.54$ & Packstone-wackestone & \\
\hline 262 & $825.54-827.65$ & Packstone-marl & Large burrows; intraclasts \\
\hline 263 & $827.65-829.07$ & Wackestone & Clay seams; moldic porosity; intraclasts \\
\hline 264 & $829.07-829.63$ & Packstone & Large burrows \\
\hline 265 & $829.63-831.00$ & Wackestone & Moldic porosity; intraclasts \\
\hline 266 & $831.00-831.30$ & Carbonaceous mudstone & (Core $89 \mathrm{R}$, Sections $5,100-113$, and $6,0-16 \mathrm{~cm}$ ) \\
\hline 267 & $831.30-831.75$ & Packstone & Moldic porosity; intraclasts \\
\hline 268 & $831.75-831.92$ & Clay & \\
\hline 269 & $831.92-832.10$ & Packstone & \\
\hline 270 & $832.10-834.68$ & Packstone & Clay seams \\
\hline 271 & $834.68-834.92$ & Clay & \\
\hline $\begin{array}{l}272 \\
273\end{array}$ & $\begin{array}{l}834.92-835.12 \\
835.12-835.57\end{array}$ & Packstone & \\
\hline 274 & $\begin{array}{l}835.12-835.57 \\
835.57-837.59\end{array}$ & $\begin{array}{l}\text { Ciay } \\
\text { Basalt }\end{array}$ & \\
\hline
\end{tabular}

rudstone-grainstone) were grouped under rudstone. Algal-mat-rich intervals and laminite were grouped together. Inaccurate estimations of abundance arise from the minimum practical resolution of the FMS tool $(0.5 \mathrm{~cm})$. Clay in recovered core material is distributed as individual fine (millimeter-thickness) seams, intervals of closely spaced fine seams, and as sharply defined centimeter-scale beds. The presence of millimeter-scale clay seams can be distinguished in the best quality FMS images because of the large resistivity contrast with their surroundings, but because of the resolution limitations of the FMS tool, the thicknesses of such seams are falsely imaged as 0.5 to 1.0 $\mathrm{cm}$. The same is probably true of the less common, thin, highly resistive, wackestone intervals and hard grounds. Bed thicknesses of $1 \mathrm{~cm}$ or less (as measured in the FMS images) were not included in the statistical analysis.

Packstone is the dominant lithology at Hole $865 \mathrm{~A}(51 \%)$, whereas wackestone, which constitutes only $3 \%$ of the logged section, was the dominant lithology recovered. Similarly, except for the dolomites at the base of the section, wackestone, wackestone-mudstone, mudstone, and laminite dominated recovery at Hole $866 \mathrm{~A}$. The grainstones and packstones that make up $25 \%$ and $20 \%$ of the section, respectively, were poorly represented in the recovered core material.

\section{SUMMARY OF LOG-CORE INTEGRATION FOR HOLE 865A}

Both core samples identified as "speleothem" and the broad range of porosities that characterize the depth interval from 140.0 to 162.0 mbsf suggest that fresh water may have been present in the formation; however, carbon isotope data are inconclusive in this regard (Sager, Winterer, Firth, et al., 1993).

The depth interval from 162.0 to 600.0 mbsf consists almost exclusively of upward-fining sequences of packstone, wackestone, and mudstone. Typically, the packstones are extremely porous and have extensive moldic porosity, and the wackestones and mudstones, with and without moldic porosity, are burrowed. Alternating intervals of rhythmic variations in resistivity and homogeneous zones are common throughout this interval. The character of the gamma, density, and velocity (not shown) logs indicates the presence of many thin layers in the variable zones. The FMS shows numerous thin layers of alternating high and low resistivities on the order of $10 \mathrm{~cm}$, with some as thin as $1 \mathrm{~cm}$.

The first traces of clays were detected in cores at about $600 \mathrm{mbsf}$, and confirmed by elevated gamma readings. Clay seams of measur- 
Table 2. Description of beds at Hole 866A (Resolution Guyot).

\begin{tabular}{|c|c|c|c|}
\hline $\begin{array}{c}\text { Bed } \\
\text { number }\end{array}$ & $\begin{array}{l}\text { Depth } \\
\text { range (mbsf) }\end{array}$ & Lithology & Description \\
\hline 1 & $195.00-206.77$ & Wackestone-packstone & Poor resolution, large hole size \\
\hline 2 & $206.77-214.22$ & Packstone & Minor moldic porosity; burrowed; induration decreases downhole \\
\hline 3 & $214.22-214.72$ & Wackestone & (Core 24R) \\
\hline 4 & $214.72-216.30$ & Packstone & Minor moldic porosity; intraclasts \\
\hline 5 & $216.30-216.84$ & Boundstone & \\
\hline 6 & $216.84-223.05$ & Packstone & Minor moldic porosity \\
\hline 7 & $223.05-223.43$ & Rudstone & \\
\hline 8 & $223.43-224.70$ & Packstone & Intraclasts; bottom $20 \mathrm{~cm}$ more porous \\
\hline 9 & $224.70-224.89$ & Mudstone & Sharp bed boundaries \\
\hline 10 & $224.89-226.32$ & Packstone-rudstone & Very porous; porosity increases downhole; minor grainstone (Core 25R) \\
\hline 11 & $226.32-227.55$ & Mudstone & \\
\hline 12 & $227.55-229.45$ & Wackestone-packstone & Minor moldic porosity \\
\hline 13 & $229.45-233.92$ & Packstone & Minor moldic porosity; burrowed (229.8) \\
\hline 14 & $233.92-235.40$ & Rudstone & \\
\hline 15 & $235.40-241.02$ & Packstone & $\begin{array}{l}\text { Minor moldic porosity; intraclasts; extensively burtowed; minor } \\
\text { grainstone (235.8?) }\end{array}$ \\
\hline 16 & $241.02-242.25$ & Mudstone & Minor moldic porosity \\
\hline 17 & $242.25-246.75$ & Wackestone-packstone & $\begin{array}{l}\text { Minor moldic porosity; vertical fracture }(247-246) \text { could be drilling- } \\
\text { related; grainstone interbeds (Core } 27 \mathrm{R})\end{array}$ \\
\hline 18 & 246.75-251.98 & Packstone & Minor moldic porosity \\
\hline 19 & $251.98-253.04$ & Rudstone-packstone & Moldic porosity \\
\hline 20 & $253.04-254.05$ & Grainstone & Moldic porosity (Core $28 \mathrm{R}$ ) \\
\hline $2 \mathrm{I}$ & $254.05-255.06$ & Rudstone & \\
\hline 22 & $255.06-258.46$ & Wackestone-packstone & $\begin{array}{l}\text { Fossiliferous; minor moldic porosity; intergranular porosity increases } \\
\text { upsection }\end{array}$ \\
\hline 23 & $258.46-260.46$ & Rudstone & \\
\hline 24 & $260.46-261.89$ & Packstone-wackestone & Packstone grading up to wackestone \\
\hline 25 & $261.89-264.40$ & Rudstone & $\begin{array}{l}\text { Intergranular porosity decreases upsection; large-scale moldic porosity; } \\
\text { intraclasts, } 263.4-264.4 \text { (Core } 29 \mathrm{R} \text { ) }\end{array}$ \\
\hline 26 & $264.40-269.16$ & Wackestone-packstone & Minor moldic porosity; extensive bioturbation \\
\hline 27 & $269.16-270.31$ & Packstone-wackestone & Minor moldic porosity; intraclasts; stylolites \\
\hline 28 & $270.31-270.74$ & Wackestone-packstone & \\
\hline 29 & $270.74-272.40$ & Rudstone & Dissolution halos \\
\hline 30 & $272.40-273.60$ & Wackestone-packstone & Moldic porosity \\
\hline 31 & $273.60-273.95$ & Packstone-grainstone & Moldic porosity \\
\hline 32 & $273.95-275.69$ & Wackestone-packstone & Moldic porosity \\
\hline 33 & $275.69-276.70$ & Packstone & Minor moldic porosity \\
\hline 34 & $276.70-277.43$ & Rudstone & Moldic porosity \\
\hline 35 & $277.43-281.80$ & Packstone-wackestone & Extensive moldic porosity; intergranular porosity decreases upsection \\
\hline 36 & $281.80-283.80$ & Wackestone & Moldic porosity (Core 31R) \\
\hline 37 & $283.80-285.77$ & Mudstone & Moldic porosity (Core 31R) \\
\hline 38 & $285.77-289.68$ & Wackestone-mudstone & Moldic porosity (Core 31R) \\
\hline 39 & $289.68-290.73$ & Packstone & Moldic porosity \\
\hline 40 & $290.73-295.00$ & Wacketone-mudstone & Very large vugs (10-12 cm); stylolites, 294.0 (Core 32R) \\
\hline 41 & $295.00-296.08$ & Wackestone & Moldic porosity \\
\hline 42 & $296.08-296.60$ & Rudstone-packstone & \\
\hline 43 & $296.60-299.20$ & Rudstone & \\
\hline 44 & $299.20-303.34$ & Packstone & Moldic porosity \\
\hline 45 & $303.34-303.80$ & Packstone-wackestone & \\
\hline 46 & $303.80-304.58$ & Rudstone & \\
\hline 47 & $304.58-305.80$ & Packstone & Moldic porosity \\
\hline 48 & $305.80-306.20$ & Packstone-wackestone & Moldic porosity \\
\hline 49 & $306.20-307.40$ & Rudstone & \\
\hline 50 & $307.40-308.86$ & Packstone & Moldic porosity; uniform intergranular porosity \\
\hline 51 & $308.86-310.15$ & Rudstone & Very porous \\
\hline 52 & $310.15-311.64$ & Packstone-rudstone & Moldic porosity \\
\hline 53 & $311.64-312.25$ & Packstone-wackestone & Moldic porosity; burrowed \\
\hline 54 & $312.25-313.80$ & Packstone & Very porous; moldic porosity; burrowed \\
\hline 55 & $313.80-315.00$ & Mudstone-wackestone & Clayey limestone; moldic porosity; minor grainstone \\
\hline 56 & $315.00-321.80$ & Packstone & $\begin{array}{l}\text { Wacke-packstone in places; mudstone intraclasts; moldic porosity } \\
\text { increases upsection; minor rudstone at } 320.0\end{array}$ \\
\hline 57 & $321.80-322.60$ & Rudstone-packstone & Minor moldic porosity \\
\hline 58 & $322.60-324.00$ & Rudstone & \\
\hline 59 & $324.00-325.30$ & Mudstone-wackestone & Intraclasts; uniform intergranular porosity; minor (Core35R) \\
\hline 60 & $325.30-325.86$ & Rudstone & Very porous \\
\hline 61 & $325.86-326.23$ & Packstone-rudstone & Moldic porosity \\
\hline 62 & $325.23-329.44$ & Packstone & Moldic porosity \\
\hline 63 & $329.44-331.40$ & Rudstone-packstone & Moldic porosity; sharp upper contact \\
\hline 64 & $331.40-332.87$ & Packstone-rudstone & Moldic porosity \\
\hline 65 & $332.87-333.37$ & Rudstone-packstone & Moldic porosity \\
\hline 66 & $333.37-334.37$ & Packstone & Moldic porosity; intraclasts \\
\hline 67 & $334.37-335.86$ & Packstone-rudstone & Moldic porosity \\
\hline 68 & $335.86-346.26$ & Mudstone & $\begin{array}{l}\text { Extreme moldic porosity; intergranular porosity increases downsection; } \\
\text { large }(2-3 \mathrm{~cm}) \text { molds to } 340.2 \text {; smaller molds }(1-2 \mathrm{~cm}) \text { in lower part } \\
\text { of section (Cores } 36 \mathrm{R}, 37 \mathrm{R}, 38 \mathrm{R})\end{array}$ \\
\hline 69 & $346.26-347.04$ & Wackestone-mudstone & Moldic porosity; mudstone intraclasts \\
\hline 70 & $347.04-347.24$ & Mudstone & Moldic porosity \\
\hline 71 & $347.24-349.00$ & Mudstone-wackestone & Small-scale $(<1 \mathrm{~cm})$ moldic porosity \\
\hline 72 & $349.00-349.30$ & Rudstone-packstone & \\
\hline 73 & $349.30-350.53$ & Rudstone-wackestone & Moldic porosity; intraclasts \\
\hline 74 & $350.53-354.13$ & Mudstone-wackestone & Minor moldic porosity \\
\hline 75 & $354.13-356.12$ & Mudstone & Minor moldic porosity \\
\hline 76 & $356.12-362.30$ & Wackestone-mudstone & Minor moldic porosity; stratified; mudstone intraclasts \\
\hline 77 & $362.30-363.58$ & Packstone & \\
\hline 78 & $363.58-363.95$ & Mudstone & Massive \\
\hline 79 & $363.95-364.63$ & Wackestone & Minor moldic porosity \\
\hline 80 & $364.63-370.40$ & Rudstone-packstone & Rudstone at base grading up to packstone; intraclasts; moldic porosity \\
\hline 81 & $370.40-370.53$ & Mudstone & Massive \\
\hline
\end{tabular}


Table 2 (continued).

\begin{tabular}{|c|c|c|c|}
\hline $\begin{array}{l}\text { Bed } \\
\text { number }\end{array}$ & $\begin{array}{l}\text { Depth } \\
\text { range (mbsf) }\end{array}$ & Lithology & Description \\
\hline 82 & $370.53-372.05$ & Packstone & Moldic porosity; intraclasts \\
\hline 83 & $372.05-373.40$ & Packstone & $\begin{array}{l}\text { Minor moldic porosity; lower intergranular porosity than Bed } 82 \text {; } \\
\text { stylolites; tension gash }\end{array}$ \\
\hline 84 & $373.40-374.88$ & Packstone-wackestone & \\
\hline 85 & $374.88-375.68$ & Wackestone-packstone & (Core 41R) \\
\hline 86 & $375.68-383.20$ & Packstone & $\begin{array}{l}\text { Minor moldic porosity; variable intergranular porosity stylolites; tension } \\
\text { gash }\end{array}$ \\
\hline 87 & $383.20-387.20$ & Wackestone-mudstone & Minor boundstone(?) at 384.5; minor moldic porosity (Core 42R) \\
\hline 88 & $387.20-388.20$ & Packstone & \\
\hline 89 & $388.20-391.67$ & Wackestone-mudstone & $\begin{array}{l}\text { Vertical fractures probably drilling-related; mudstone intraclasts (391- } \\
391.5 \text { ); moldic porosity }\end{array}$ \\
\hline 90 & $391.67-392.77$ & Packstone & Mudstone intraclasts \\
\hline 91 & $392.77-392.96$ & Wackestone-mudstone & \\
\hline 92 & $392.96-393.90$ & Mudstone-wackestone & Moldic porosity (Core 43R) \\
\hline 93 & $393.90-396.09$ & Wackestone-mudstone & Massive; minor moldic porosity (Core 44R) \\
\hline 94 & $396.09-397.30$ & Packstone & Moldic porosity; intraclasts \\
\hline 95 & $397.30-399.80$ & Rudstone & Vertical fracture probably drilling-related \\
\hline 96 & $399.80-399.97$ & Mudstone & Massive \\
\hline 97 & $399.97-400.87$ & Packstone & $\begin{array}{l}\text { Intraclasts; stylolites } \\
\text { Massive. }\end{array}$ \\
\hline 98 & $400.87-401.24$ & Mudstone & $\begin{array}{l}\text { Massive } \\
\text { Larce-scale }(\sim 4 \mathrm{~cm}) \text { moldic porosity: mudstone intraclasts: vertical }\end{array}$ \\
\hline 99 & $401.24-403.09$ & Packstone & $\begin{array}{l}\text { Large-scale }(\sim 4 \mathrm{~cm}) \text { moldic porosity; mudstone intraclasts; vertical } \\
\text { fractures probably drilling related }\end{array}$ \\
\hline 100 & $403.09-403.60$ & Mudstone & \\
\hline 101 & $403.60-404.00$ & Packstone & \\
\hline 102 & $404.00-407.40$ & Mudstone & Minor moldic porosity; burrowed(?) \\
\hline 103 & $407.40-409.80$ & Mudstone & Variable cementation; moldic porosity \\
\hline 104 & $409.80-411.90$ & Packstone & Minor moldic porosity; stylolites \\
\hline 105 & $411.90-413.60$ & Mudstone-wackestone & $\begin{array}{l}\text { Moldic porosity } \\
\text { Fxtreme moldich }\end{array}$ \\
\hline 106 & $413.60-430.24$ & Mudstone-wackestone & $\begin{array}{l}\text { Extreme moldic porosity; intergranular porosity highly variable; stylolites } \\
\text { (Cores } 46 \mathrm{R}, 47 \mathrm{R})\end{array}$ \\
\hline 107 & $430.24-431.40$ & Wackestone-mudstone & \\
\hline 108 & $431.40-442.01$ & Packstone & $\begin{array}{l}\text { Very porous with moldic porosity } 433.0 \text { to top of section; middle part less } \\
\text { porous; very porous at base }\end{array}$ \\
\hline 109 & $442.01-443.50$ & Wackestone-mudstone & \\
\hline 110 & $443.50-444.00$ & Grainstone & (Core 48R) \\
\hline 111 & $444.00-444.83$ & Wackestone-mudstone & \\
\hline 112 & $444.83-449.29$ & Packstone & Variable induration \\
\hline 113 & & Marl & Very porous at base; mudstone(?) intraclasts (Core 50R) \\
\hline $\begin{array}{l}114 \\
115\end{array}$ & $\begin{array}{l}450.10-456.53 \\
456.53-457.03\end{array}$ & $\begin{array}{l}\text { Packstone } \\
\text { Marl }\end{array}$ & \\
\hline 116 & $457.03-457.60$ & $\begin{array}{l}\text { Marl } \\
\text { Packstone }\end{array}$ & \\
\hline 117 & $457.60-458.48$ & Marl & \\
\hline 118 & $458.48-465.10$ & Packstone & $\begin{array}{l}\text { Moldic porosity; several well-cemented intervals; minor marl interbeds; } \\
\text { minor grainstone }\end{array}$ \\
\hline 119 & $465.10-466.44$ & Packstone & Moldic porosity \\
\hline 120 & $466.44-466.80$ & Grainstone & \\
\hline 121 & $466.80-467.33$ & Packstone & Moldic porosity \\
\hline 122 & $467.33-467.55$ & Wackestone & \\
\hline 123 & $467.55-468.38$ & Packstone & Moldic porosity; mudstone intraclasts or burrow fillings \\
\hline 124 & $468.38-468.86$ & Grainstone-packstone & Minor moldic porosity (Core 51R) \\
\hline 125 & $468.86-469.03$ & Packstone & Moldic porosity; intraclasts (Core 51R) \\
\hline 126 & $469.03-469.39$ & Wackestone & \\
\hline 127 & $469.39-470.33$ & $\begin{array}{l}\text { Packstone } \\
\text { Wackestone }\end{array}$ & \\
\hline 128 & $470.33-471.00$ & $\begin{array}{l}\text { Wackestone } \\
\text { Packstone }\end{array}$ & Highly variable intergranular porosity; stylolites \\
\hline $\begin{array}{l}129 \\
130\end{array}$ & $\begin{array}{l}471.00-472.63 \\
472.63-472.95\end{array}$ & $\begin{array}{l}\text { Packstone } \\
\text { Packstone-grainstone }\end{array}$ & \\
\hline $\begin{array}{l}130 \\
131\end{array}$ & $472.95-473.44$ & $\begin{array}{l}\text { Packstone-grainstone } \\
\text { Packstone-wackestone }\end{array}$ & Moldic porosity; vertical fractures \\
\hline $\begin{array}{l}131 \\
132\end{array}$ & $473.44-473.84$ & Wackestone-packstone & \\
\hline 133 & $473.84-476.22$ & Packstone-wackestone & Packstone with wackestone interbeds; very minor moldic porosity \\
\hline 134 & $476.22-474.56$ & Packstone-grainstone & Stylolites \\
\hline 135 & $474.56-477.80$ & Packstone & \\
\hline 136 & $477.80-478.58$ & Packstone-grainstone & Stylolites \\
\hline 137 & $478.58-479.00$ & Packstone & \\
\hline 138 & $479.00-479.47$ & Packstone-wackestone & Stylolites; intraclasts \\
\hline 139 & $479.47-480.65$ & Packstone & Intraclasts; vertical fractures \\
\hline $\begin{array}{l}140 \\
141\end{array}$ & $\begin{array}{l}480.65-481.05 \\
481.05-481.40\end{array}$ & $\begin{array}{l}\text { Wackestone-packstone } \\
\text { Packstone }\end{array}$ & \\
\hline $\begin{array}{l}141 \\
142\end{array}$ & $\begin{array}{l}481.05-481.40 \\
481.40-481.60\end{array}$ & $\begin{array}{l}\text { Packstone } \\
\text { Wackestone-grainstone }\end{array}$ & Intraclasts \\
\hline $\begin{array}{l}142 \\
143\end{array}$ & $481.60-482.82$ & Packstone & Moldic porosity; stylolites \\
\hline 144 & $482.82-482.98$ & Wackestone & \\
\hline 145 & $482.98-484.60$ & Packstone & Marly interval (483.6-483.8); stylolites \\
\hline 146 & $484.60-485.48$ & Marl & Stylolites \\
\hline 147 & $485.48-486.83$ & Packstone & Minor $(<2 \mathrm{~cm})$ wackestone interval; stylolites \\
\hline 148 & $486.83-486.96$ & Wackestone & Massive \\
\hline 149 & $486.96-487.82$ & Wackestone-packstone & Minor moldic porosity \\
\hline 150 & $487.82-489.32$ & Packstone-wackestone & Moldic porosity; vertical fracture (drilling related) \\
\hline 151 & $489.32-490.43$ & Packstone & Very porous; moldic porosity \\
\hline 152 & $490.43-490.60$ & Wackestone & \\
\hline 153 & $490.60-490.83$ & Wackestone-packstone & \\
\hline 154 & $490.83-491.57$ & Packstone & \\
\hline 155 & $491.57-492.50$ & Wackestone-packstone & \\
\hline 156 & $492.50-494.05$ & Packstone-wackestone & \\
\hline $\begin{array}{l}157 \\
158\end{array}$ & $\begin{array}{l}494.05-494.20 \\
494.20-495.56\end{array}$ & $\begin{array}{l}\text { Wackestone } \\
\text { Packstone }\end{array}$ & \\
\hline $\begin{array}{l}158 \\
159\end{array}$ & $\begin{array}{l}494.20-495.56 \\
495.56-495.80\end{array}$ & $\begin{array}{l}\text { Packstone } \\
\text { Wackestone }\end{array}$ & $\begin{array}{l}\text { Moldic porosity } \\
\text { Minor moldic porosity }\end{array}$ \\
\hline 160 & $495.80-496.60$ & Packstone & Moldic porosity; stylolites \\
\hline 161 & $496.60-496.80$ & Wackestone & \\
\hline 162 & $496.80-497.22$ & Packstone & Minor moldic porosity; intraclasts \\
\hline
\end{tabular}


Table 2 (continued).

\begin{tabular}{|c|c|c|c|}
\hline $\begin{array}{c}\text { Bed } \\
\text { number }\end{array}$ & $\begin{array}{c}\text { Depth } \\
\text { range (mbsf) }\end{array}$ & Lithology & Description \\
\hline 163 & $497.22-497.40$ & Wackestone & \\
\hline 164 & $497.40-498.95$ & Packstone & Minor moldic porosity; intraclast \\
\hline 165 & $498.95-500.97$ & Wackestone & Minor grainstone; minor moldic porosity (Core $54 \mathrm{R}$ ) \\
\hline 166 & $500.97-501.49$ & Wackestone-packstone & \\
\hline 167 & $501.49-501.75$ & Packstone & Moldic porosity \\
\hline 168 & $501.75-502.64$ & Wackestone-packstone & Minor grainstone \\
\hline 169 & $502.64-503.70$ & Packstone & \\
\hline 170 & $503.70-503.80$ & Wackestone & \\
\hline 171 & $503.80-504.09$ & Packstone & Moldic porosity \\
\hline 172 & $504.09-505.40$ & Packstone-wackestone & \\
\hline 173 & $505.40-506.40$ & Packstone & \\
\hline 174 & $506.40-508.05$ & Packstone-wackestone & Minor grainstone \\
\hline 175 & $508.05-508.51$ & Packstone-grainstone & (Core 55R) \\
\hline 176 & $508.51-509.55$ & Packstone & \\
\hline 177 & $509.55-509.80$ & Wackestone & \\
\hline 178 & $509.80-511.18$ & Packstone & \\
\hline 179 & $511.18-512.58$ & Packstone-wackestone & \\
\hline 180 & $512.58-514.68$ & Packstone & Moldic porosity \\
\hline 181 & $514.68-515.04$ & Packstone-wackestone & \\
\hline 182 & $515.04-517.07$ & Packstone & Moldic porosity; stylolites (Core 56R) \\
\hline 183 & $517.07-517.63$ & Wackestone & \\
\hline 184 & $517.63-519.20$ & Packstone-wackestone & Minor moldic porosity; minor grainstone \\
\hline 185 & $519.20-520.18$ & Packstone & \\
\hline 186 & $520.18-520.35$ & Packstone-wackestone & \\
\hline 187 & $520.35-520.57$ & Wackestone & \\
\hline 188 & $520.57-522.86$ & Packstone & Moldic porosity limited to distinct intervals \\
\hline 189 & $522.86-523.08$ & Packstone-grainstone & \\
\hline 190 & $523.08-523.91$ & Packstone-wackestone & \\
\hline 191 & $523.91-525.37$ & Packstone & Moldic porosity; intraclasts \\
\hline 192 & $525.37-526.24$ & Packstone-grainstone & 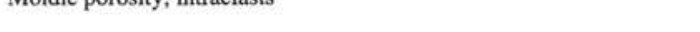 \\
\hline 193 & $526.24-526.63$ & Packstone-wackestone & \\
\hline 194 & $526.63-527.95$ & Packstone & Intraclasts at base (probably of underlying Unit 195); stylolites \\
\hline 195 & $527.95-529.11$ & Packstone-grainstone & \\
\hline 196 & $529.11-531.44$ & Packstone-wackestone & \\
\hline 197 & $531.44-532.69$ & Packstone-grainstone & \\
\hline 198 & $532.69-534.12$ & Packstone & Minor moldic porosity; intraclasts \\
\hline 199 & $534.12-534.31$ & Packstone-grainstone & (Core 58R) \\
\hline 200 & $534.31-534.57$ & Packstone & Intraclasts \\
\hline 201 & $534.57-534.94$ & Packstone-grainstone & \\
\hline 202 & $534.94-535.53$ & Grainstone & \\
\hline 203 & $535.53-537.93$ & Wackestone-rudstone & \\
\hline 204 & $537.93-541.60$ & Packstone-grainstone & \\
\hline 205 & $541.60-542.07$ & Packstone & \\
\hline 206 & $542.07-542.55$ & Packstone-grainstone & \\
\hline 207 & $542.55-544.14$ & Packstone & Intraclasts \\
\hline 208 & $544.14-544.50$ & Wackestone & \\
\hline 209 & $544.50-544.88$ & Wackestone-packstone & \\
\hline 210 & $544.88-546.62$ & Packstone & \\
\hline 211 & $546.62-546.77$ & Wackestone & \\
\hline 212 & $546.77-549.00$ & Packstone & Variable cementation \\
\hline 213 & $549.00-550.20$ & Packstone-wackestone & \\
\hline 214 & $550.20-551.20$ & Packstone & Moldic porosity \\
\hline 215 & $551.20-551.40$ & Wackestone & \\
\hline 216 & $551.40-552.50$ & Packstone & \\
\hline 217 & $552.50-553.09$ & Wackestone-packstone & \\
\hline 218 & $553.09-553.33$ & Packstone-grainstone & (Core 60R) \\
\hline 219 & $553.33-556.42$ & Packstone-wackestone & Packstone with $\sim 2-\mathrm{cm}$ intervals of wackestone; minor moldic porosity \\
\hline 220 & $556.42-560.03$ & Packstone & Moldic porosity; intraclasts; dipping basal contact \\
\hline 221 & $560.03-560.20$ & Wackestone & \\
\hline 222 & $560.20-563.18$ & Packstone & Moldic porosity; burrows(?) \\
\hline 223 & $563.18-563.54$ & Packstone-grainstone & \\
\hline 224 & $563.54-563.84$ & Grainstone & \\
\hline 225 & $563.84-564.16$ & Packstone-grainstone & \\
\hline 226 & $564.16-566.20$ & Packstone & Vertical fracture or burrow (564.4) \\
\hline 227 & $566.20-566.35$ & Packstone-wackestone & \\
\hline 228 & $566.35-567.80$ & Packstone & \\
\hline 229 & $567.80-567.91$ & Wackestone & \\
\hline 230 & $567.91-569.23$ & Packstone & Moldic porosity; top is burrowed \\
\hline 231 & $569.23-569.80$ & Packstone-grainstone & \\
\hline 232 & $569.80-570.36$ & Packstone-wackestone & \\
\hline 233 & $570.36-573.79$ & Packstone-grainstone & \\
\hline 234 & $573.79-577.98$ & Packstone-wackestone & \\
\hline 235 & $577.98-578.88$ & Rudstone & \\
\hline 236 & $578.88-579.98$ & Wackestone & Minor moldic porosity \\
\hline 237 & $579.98-582.47$ & Wackestone-packstone & Numerous intraclasts and/or burrows; minor moldic porosity \\
\hline 238 & $582.47-584.89$ & Packstone-wackestone & Moldic porosity; intraclasts \\
\hline 239 & $584.89-585.82$ & Wackestone & \\
\hline 240 & $585.82-586.20$ & Grainstone-packstone & Minor moldic porosity; burrowed (Core 63R) \\
\hline 241 & $586.20-587.42$ & Rudstone-wackestone & \\
\hline 242 & $587.42-589.25$ & Grainstone-packstone & Clay seams \\
\hline 243 & $589.25-590.17$ & Packstone & Clay seams \\
\hline 244 & $590.17-591.25$ & Grainstone-packstone & \\
\hline 245 & $591.25-592.00$ & Packstone & Moldic porosity; intraclasts \\
\hline 246 & $592.00-593.80$ & Grainstone & Minor moldic porosity; stylolites \\
\hline 247 & $593.80-595.72$ & Packstone & Minor moldic porosity; intraclasts; stylolites (Core 64R) \\
\hline 248 & $595.72-596.03$ & Wackestone & (Core 64R) \\
\hline 249 & $596.03-596.81$ & Packstone-wackestone & \\
\hline 250 & $596.81-598.05$ & Grainstone & Moldic porosity \\
\hline 251 & $598.05-599.85$ & Grainstone & Minor moldic porosity \\
\hline
\end{tabular}


Table 2 (continued).

\begin{tabular}{|c|c|c|c|}
\hline $\begin{array}{c}\text { Bed } \\
\text { number }\end{array}$ & $\begin{array}{c}\text { Depth } \\
\text { range (mbsf) }\end{array}$ & Lithology & Description \\
\hline 252 & $599.85-600.72$ & Packstone & Dissolution halos \\
\hline 253 & $600.72-600.97$ & Wackestone & (Core 65R) \\
\hline 254 & $600.97-606.22$ & Packstone & Minor moldic porosity \\
\hline 255 & $606.22-607.37$ & Packstone-grainstone & \\
\hline 356 & $607.37-608.30$ & Grainstone & Intraclasts \\
\hline 257 & $608.30-609.82$ & Packstone-wackestone & Intraclasts \\
\hline 258 & $609.82-610.33$ & Packstone & \\
\hline $\begin{array}{l}259 \\
260\end{array}$ & $\begin{array}{l}610.33-611.27 \\
611.27-612.58\end{array}$ & & \\
\hline $\begin{array}{l}260 \\
261\end{array}$ & $612.58-613.16$ & $\begin{array}{l}\text { Packstone-rudstone } \\
\text { Wackestone }\end{array}$ & \\
\hline 262 & $613.16-617.15$ & Packstone & Minor grainstone; minor moldic porosity \\
\hline 263 & $617.15-618.13$ & Wackestone & \\
\hline 264 & $618.13-621.66$ & Packstone & Moldic porosity; numerous intraclasts \\
\hline 265 & $621.66-621.80$ & Wackestone & \\
\hline 266 & $621.80-625.20$ & Packstone & Moldic porosity; intraclasts \\
\hline 267 & $625.20-625.68$ & Packstone-grainstone & (Core 67R) \\
\hline 268 & $625.68-625.83$ & Grainstone & \\
\hline $\begin{array}{l}269 \\
270\end{array}$ & $625.83-627.17$ & $\begin{array}{l}\text { Packstone } \\
\text { Grainstone }\end{array}$ & \\
\hline $\begin{array}{l}270 \\
271\end{array}$ & $\begin{array}{l}627.17-627.30 \\
627.30-629.03\end{array}$ & $\begin{array}{l}\text { Grainstone } \\
\text { Wackestone-packstone }\end{array}$ & (Core 68R) \\
\hline 272 & $629.03-629.95$ & Packstone & \\
\hline 273 & $629.95-632.10$ & Packstone-wackestone & Minor moldic porosity \\
\hline 274 & $632.10-634.80$ & Packstone & Moldic porosity; stylolites \\
\hline 275 & $634.80-635.39$ & Packstone-grainstone & \\
\hline 276 & $635.39-637.20$ & Packstone & \\
\hline 277 & $637.20-638.04$ & Packstone-grainstone & \\
\hline 278 & $638.04-640.48$ & Packstone & \\
\hline 279 & $640.48-641.11$ & Wackestone-packstone & Intraclasts \\
\hline $\begin{array}{l}280 \\
281\end{array}$ & $\begin{array}{l}641.11-642.27 \\
642.27-643.46\end{array}$ & $\begin{array}{l}\text { Packstone-wackestone } \\
\text { Packstone-wackestone }\end{array}$ & \\
\hline 282 & $643.46-648.00$ & Packstone & Burrowed; burrow fillings both light (calcite) and dark (clay, pyrite?) \\
\hline 283 & $648.00-649.06$ & Wackestone-mudstone & Contact with Unit 282 is gradational \\
\hline 284 & $649.06-649.27$ & Mudstone & \\
\hline 285 & $649.27-649.54$ & Laminites & Clay seams \\
\hline 286 & $649.54-650.27$ & Mudstone & $\begin{array}{l}\text { Muds and clays, some stratified, some well-mixed; this is a single, } \\
\text { upward-fining unit }\end{array}$ \\
\hline 287 & $650.27-650.53$ & Laminites & Minor mudstone \\
\hline 288 & $650.53-652.45$ & Mudstone & Burrowed \\
\hline 289 & $652.45-652.73$ & Packstone & Cementation highly variable \\
\hline 290 & $652.73-653.40$ & Mudstone & Minor moldic porosity; clay seams; sharp basal contact \\
\hline 291 & $653.40-654.93$ & Packstone & Minor moldic porosity \\
\hline 292 & $654.93-655.48$ & Wackestone-mudstone & Minor moldic porosity (Core 70R) \\
\hline 293 & $655.48-655.57$ & Mudstone-grainstone & (Core 70R) \\
\hline 294 & $655.57-655.90$ & Mudstone & Massive \\
\hline 295 & $655.90-656.32$ & Mudstone & Clay seams \\
\hline 296 & $656.32-657.89$ & Packstone & \\
\hline 297 & $657.89-658.23$ & Wackestone-mudstone & Intraclast \\
\hline $\begin{array}{l}298 \\
299\end{array}$ & & $\begin{array}{l}\text { Mudstone-wackestone } \\
\text { Packstone }\end{array}$ & \\
\hline 300 & $\begin{array}{l}658.48-661.50 \\
661.50-663.94\end{array}$ & $\begin{array}{l}\text { Packstone } \\
\text { Mudstone }\end{array}$ & \\
\hline 301 & $663.94-663.96$ & Clay & \\
\hline 302 & $663.96-665.24$ & Mudstone & Mudstone laminites with clay (Core 71R) \\
\hline 303 & $665.24-665.85$ & Wackestone & \\
\hline 304 & $665.85-668.25$ & Packstone-wackestone & Minor moldic porosity; intraclasts \\
\hline 305 & $668.25-669.85$ & Packstone & Very porous; bimodal moldic porosity \\
\hline 306 & $669.85-671.90$ & Wackestone-mudstone & $\begin{array}{l}\text { Interbedded wackestones and mudstones; clay seams; stylolites (Core } \\
\quad 72 \mathrm{R})\end{array}$ \\
\hline 307 & $671.90-673.37$ & Packstone & Moldic porosity \\
\hline 308 & $673.37-674.50$ & Mudstone-wackestone & Burrowed \\
\hline 309 & $674.50-674.60$ & Mudstone & Massive \\
\hline 310 & $674.60-675.13$ & Rudstone & \\
\hline 311 & $675.13-675.70$ & Packstone & Moldic porosity \\
\hline 312 & $675.70-676.20$ & Packstone-wackestone & (Core 73R) \\
\hline 313 & $676.20-676.43$ & Mudstone & Mudstone laminites with clay \\
\hline 314 & $676.43-677.50$ & Rudstone & \\
\hline 315 & $677.50-676.60$ & Grainstone & Mudstone(?) intraclasts; stylolites (Core 73R) \\
\hline 316 & $676.60-678.75$ & Mudstone & Clay seam \\
\hline 317 & $678.75-680.11$ & Packstone & Moldic porosity; intraclasts \\
\hline 318 & $680.11-682.20$ & Rudstone & Grainstone interbeds \\
\hline 319 & $682.20-683.45$ & Grainstone & Moldic porosity; cross-bedding \\
\hline 320 & $683.45-687.63$ & Rudstone & Grading downward to packstone-grainstone \\
\hline 321 & $687.63-688.03$ & Grainstone & \\
\hline 322 & $688.03-690.36$ & Wackestone-mudstone & Minor moldic porosity \\
\hline 323 & $690.36-690.88$ & Mudstone & Moldic porosity \\
\hline 324 & $690.88-691.84$ & Wackestone-mudstone & Moldic porosity \\
\hline 325 & $691.84-694.97$ & Grainstone & $\begin{array}{l}\text { Fine- to medium-grained; downward-fining; thinly bedded to massive; } \\
\text { clay seams in top of section; cross-bedding (Core 75R) }\end{array}$ \\
\hline 326 & $694.97-695.79$ & Grainstone & Clay seams; burrowed; slumps(?) \\
\hline 327 & $695.79-700.81$ & Grainstone & $\begin{array}{l}\text { Minor rudstone; minor packstone intervals; minor moldic porosity (Core } \\
75 \mathrm{R} \text { ) }\end{array}$ \\
\hline 328 & $700.81-702.58$ & Rudstone & \\
\hline 329 & $702.58-706.90$ & Grainstone & Medium-fine-grained; minor packstone \\
\hline 330 & $706.90-709.25$ & Rudstone-grainstone & \\
\hline 331 & $709.25-710.46$ & Grainstone & Medium-fine-grained \\
\hline 332 & $710.46-717.34$ & Wackestone-rudstone & Minor grainstone \\
\hline 333 & $717.34-721.83$ & Grainstone & $\begin{array}{l}\text { Medium- to coarse-grained; minor moldic porosity; well-cemented } \\
\text { intervals }\end{array}$ \\
\hline 334 & $721.83-732.33$ & Grainstone & Coarse-grained; well-cemented \\
\hline
\end{tabular}


Table 2 (continued).

\begin{tabular}{|c|c|c|c|}
\hline $\begin{array}{c}\text { Bed } \\
\text { number }\end{array}$ & $\begin{array}{l}\text { Depth } \\
\text { range (mbsf) }\end{array}$ & Lithology & Description \\
\hline 335 & $732.33-725.33$ & Grainstone & Medium- to very fine-grained \\
\hline 336 & $725.33-729.06$ & Grainstone & $\begin{array}{l}\text { Medium-grained; bioturbation (or slumping?); minor moldic porosity; } \\
\text { variable cementation }\end{array}$ \\
\hline 337 & $729.06-729.73$ & Grainstone & Well-sorted, medium-grained; intraclasts (Core 79R) \\
\hline 338 & $729.73-732.82$ & Grainstone & Medium-fine-grained; moldic porosity; intraclasts at base (Core 79R) \\
\hline 339 & $732.82-741.60$ & Grainstone & $\begin{array}{l}\text { Coarse- to medium-grained; upward-fining; clay seams; packstone } \\
\text { interbeds; moldic porosity }\end{array}$ \\
\hline 340 & $741.60-743.50$ & Grainstone-rudstone & \\
\hline 341 & $743.50-747.00$ & Rudstone & \\
\hline 342 & $747.00-748.96$ & Rudstone-grainstone & \\
\hline 343 & $748.96-752.61$ & Rudstone & \\
\hline 344 & $752.61-756.39$ & Grainstone & Minor packstone; upward-fining; moldic porosity \\
\hline 345 & $756.39-757.23$ & Rudstone & \\
\hline 346 & $757.23-758.05$ & Packstone-rudstone & Moldic porosity; intraclasts \\
\hline 347 & $758.05-760.32$ & Wackestone & \\
\hline 348 & $760.32-763.23$ & Rudstone & \\
\hline 349 & $763.23-769.63$ & Grainstone & $\begin{array}{l}\text { Minor packstone and wackestone intervals; moldic } \\
\text { porosity; intraclasts (Core } 82 \mathrm{R} \text { ) }\end{array}$ \\
\hline 350 & $769.63-770.25$ & Wackestone & \\
\hline 351 & $770.25-772.90$ & Rudstone & \\
\hline 352 & $772.90-774.45$ & Grainstone & Downward-fining \\
\hline 353 & $774.45-778.27$ & Rudstone-grainstone & \\
\hline 354 & $778.27-781.04$ & Grainstone & Minor wackestone \\
\hline 355 & $781.04-786.47$ & Rudstone-grainstone & \\
\hline 356 & $786.47-788.18$ & Packstone-grainstone & Intraclasts; dissolution halos \\
\hline 357 & $788.18-788.21$ & Wackestone & \\
\hline 358 & $788.21-790.75$ & Grainstone-packstone & \\
\hline 359 & $790.75-790.87$ & Mudstone & Calcrete(?); very well-cemented; sharp upper contact \\
\hline 360 & $790.87-792.70$ & Packstone & Very porous \\
\hline 361 & $792.70-794.30$ & Wackestone & \\
\hline 362 & $794.30-795.11$ & Packstone & (Core 85R) \\
\hline 363 & $795.11-796.26$ & Packstone-wackestone & (Core $85 \mathrm{R}$ ) \\
\hline 364 & $796.26-796.80$ & Rudstone & \\
\hline 365 & $796.80-797.78$ & Rudstone-grainstone & \\
\hline 366 & $797.78-798.10$ & Wackestone & Interbedded packstone and algal mat; clay intervals \\
\hline 367 & $798.10-798.84$ & Mudstone & Massive \\
\hline 368 & $798.84-801.80$ & Wackestone & Interbedded packstone and algal mat; distinct bedding \\
\hline 369 & $801.80-805.73$ & Packstone & Downward-fining; moldic porosity; intraclasts \\
\hline 370 & $805.73-806.60$ & Wackestone & Interbedded algal mat and wackestone; clay intervals at base of section \\
\hline 371 & $806.60-807.63$ & Mudstone & Minor wackestone; intraclasts at base (Core $86 \mathrm{R}$ ) \\
\hline 372 & $807.63-809.70$ & Wackestone & Finely bedded packstones and algal mat; minor moldic porosity \\
\hline 373 & $809.70-811.35$ & Packstone & Intraclasts \\
\hline 374 & $811.35-812.59$ & Packstone-wackestone & Laminated with algal mat \\
\hline 375 & $812.59-813.93$ & Rudstone-wackestone & Moldic porosity; intraclasts \\
\hline 376 & $813.93-814.73$ & Packstone-wackestone & Laminated with algal mat (Core 87R) \\
\hline 377 & $814.73-818.41$ & Packstone & $\begin{array}{l}\text { Downward-fining; minor intervals of grainstone and rudstone; moldic } \\
\text { porosity (Core } 87 \mathrm{R} \text { ) }\end{array}$ \\
\hline 378 & $818.41-819.49$ & Packstone & Coarse-grained; well-cemented interval at 819.2 \\
\hline 379 & $819.49-821.60$ & Packstone & Small vugs at base; intraclasts \\
\hline 380 & $821.60-822.09$ & Packstone & Grainstone intervals and minor wackestone intervals \\
\hline 381 & $822.09-822.53$ & Wackestone & \\
\hline 382 & $822.53-823.24$ & Wackestone & Interbeds of clay and wackestone \\
\hline 383 & $823.24-823.46$ & Clay & \\
\hline 384 & $823.46-824.43$ & Marl & \\
\hline 385 & $824.43-824.97$ & Marl & Burrowed \\
\hline 386 & $824.97-826.64$ & Packstone-wackestone & $\begin{array}{l}\text { With algal mat; numerous clay intervals; clay increases downsection } \\
\text { (Core } 88 \mathrm{R} \text { ) }\end{array}$ \\
\hline 387 & $826.64-827.93$ & Wackestone-rudstone & \\
\hline 388 & $827.93-828.24$ & Packstone & High intergranular porosity \\
\hline 389 & $828.24-829.65$ & Algal mat & Interbedded algal mat, mudstone, minor packstone; clay seams \\
\hline 390 & $829.65-831.82$ & Packstone & $\begin{array}{l}\text { Numerous rounded clay and limestone intraclasts; limestone intraclasts } \\
\text { concentrated at base }(831.63-831.82)\end{array}$ \\
\hline 391 & $831.82-831.99$ & Clay & \\
\hline 392 & $831.99-832.70$ & Wackestone & Some laminites (Core 89R) \\
\hline 393 & $832.70-832.76$ & Clay & (Core 89R) \\
\hline 394 & $832.76-833.38$ & Wackestone & Some laminites (Core 89R) \\
\hline 395 & $833.38-834.09$ & Packstone & (Core 89R) \\
\hline 396 & $834.09-834.95$ & Packstone-grainstone & Intraclasts (Core 89R) \\
\hline 397 & $834.95-835.48$ & Wackestone-grainstone & \\
\hline 398 & $835.48-836.00$ & Laminites & \\
\hline 399 & $836.00-836.08$ & Clay & \\
\hline 400 & $836.08-836.54$ & Wackestone-grainstone & \\
\hline 401 & $836.54-836.96$ & Packstone & Intraclasts \\
\hline 402 & $836.96-837.09$ & Wackestone & \\
\hline 403 & $837.09-837.52$ & Laminites & \\
\hline 404 & $837.52-838.70$ & Wackestone & \\
\hline 405 & $838.70-839.00$ & Rudstone & \\
\hline 406 & $839.00-839.76$ & Packstone-grainstone & Minor intraclasts \\
\hline 407 & $839.76-839.98$ & Wackestone-grainstone & \\
\hline 408 & $839.98-840.19$ & Clay seams & \\
\hline 409 & $840.19-841.00$ & Mudstone & \\
\hline 410 & $841.00-841.33$ & Clay & Clay seams and laminites \\
\hline 411 & $841.33-842.55$ & Packstone-wackestone & \\
\hline 412 & $842.55-842.65$ & Clay & Clay seams and laminites \\
\hline 413 & $842.65-843.32$ & Packstone & \\
\hline 414 & $843.32-843.87$ & Packstone-wackestone & \\
\hline 415 & $843.87-844,28$ & Rudstone & \\
\hline 416 & $844.28-844.40$ & Wackestone & \\
\hline
\end{tabular}


Table 2 (continued).

\begin{tabular}{|c|c|c|c|}
\hline $\begin{array}{c}\text { Bed } \\
\text { number }\end{array}$ & $\begin{array}{l}\text { Depth } \\
\text { range (mbsf) }\end{array}$ & Lithology & Description \\
\hline 417 & $844.40-844.53$ & Grainstone & \\
\hline 418 & $844.53-845.13$ & Wackestone & \\
\hline 419 & $845.13-845.22$ & Clay & \\
\hline 420 & $845.22-845.71$ & $\begin{array}{l}\text { Wackestone } \\
\text { Packstone }\end{array}$ & \\
\hline 421 & $845.71-846.49$ & $\begin{array}{l}\text { Packstone } \\
\text { Wackestone-grainstone }\end{array}$ & \\
\hline $\begin{array}{l}422 \\
423\end{array}$ & $\begin{array}{l}846.49-847.40 \\
847.40-847.82\end{array}$ & $\begin{array}{l}\text { Wackestone-grainstone } \\
\text { Wackestone }\end{array}$ & \\
\hline $\begin{array}{l}423 \\
424\end{array}$ & $847.82-848.80$ & $\begin{array}{l}\text { Wackestone } \\
\text { Packstone }\end{array}$ & \\
\hline 425 & $848.80-849.48$ & Wackestone & \\
\hline 426 & $849.48-849.68$ & Clay & \\
\hline 427 & $849.68-852.02$ & Wackestone & Minor packstone \\
\hline 428 & $852.02-853.34$ & Clay & $\begin{array}{l}\text { Clay intervals, algal mat, laminites; minor mudstone or wackestone } \\
\text { intervals }\end{array}$ \\
\hline 429 & $853.34-858.03$ & Grainstone & $\begin{array}{l}\text { Minor wackestone and mudstone intervals; clay seams and laminite } \\
\text { intervals }\end{array}$ \\
\hline 430 & $858.03-859.53$ & Packstone & Minor grainstone (Core 91R) \\
\hline 431 & $859.53-860.59$ & Grainstone & \\
\hline 432 & $860.59-861.38$ & Packstone-grainstone & \\
\hline 433 & $861.38-862.60$ & Wackestone & (Core 92R) \\
\hline 434 & $862.60-863.54$ & Grainstone & Coarse-grained; packstone intervals \\
\hline 435 & $863.54-864.80$ & Grainstone & Fine-grained; sharp increase in porosity \\
\hline 436 & $864.80-865.25$ & Grainstone & Coarse grainstones; minor packstone \\
\hline 437 & $865.25-866.38$ & Wackestone & \\
\hline 438 & $866.38-869.44$ & Packstone-grainstone & \\
\hline 439 & $869.44-870.20$ & Wackestone & \\
\hline 440 & $\begin{array}{l}870.20-870.51 \\
870.51-871.23\end{array}$ & Wackestone-rudstone & \\
\hline $\begin{array}{l}441 \\
442\end{array}$ & $\begin{array}{l}870.51-871.23 \\
871.23-871.46\end{array}$ & $\begin{array}{l}\text { Wackestone } \\
\text { Rudstone }\end{array}$ & \\
\hline 443 & $871.46-874.50$ & Wackestone-packstone & \\
\hline 444 & $874.50-875.57$ & Grainstone & \\
\hline 445 & $875.57-876.34$ & Wackestone & \\
\hline 446 & $876.34-881.66$ & Rudstone & \\
\hline 447 & $881.66-882.03$ & Wackestone-rudstone & \\
\hline 448 & $882.03-882.30$ & Rudstone & \\
\hline 449 & $882.30-890.16$ & Grainstone & $\begin{array}{l}\text { Grades downward into grainstone-packstone; moldic porosity evident in } \\
\text { interval } 886.15-886.55 \text { (Core } 94 \mathrm{R} \text { ) }\end{array}$ \\
\hline 450 & $890.16-891.97$ & Rudstone-packstone & \\
\hline 451 & $891.97-893.48$ & Packstone-grainstone & \\
\hline 452 & $893.48-896.36$ & Packstone & Grades upward to rudstone-grainstone \\
\hline 453 & $896.36-897.06$ & Packstone-grainstone & \\
\hline 454 & $897.06-898.00$ & Rudstone-grainstone & \\
\hline 455 & $898.00-899.10$ & Wackestone-grainstone & Wackestone grading upward to grainstone \\
\hline 456 & $899.10-901.60$ & Wackestone-packstone & (Core 96R) \\
\hline 457 & $901.60-909.53$ & Packstone-rudstone & $\begin{array}{l}\text { Alternating beds of packstone and rudstone; boundaries gradational; } \\
\text { minor wackestone }\end{array}$ \\
\hline 458 & $909.53-909.89$ & Grainstone & \\
\hline 459 & $909.89-910.87$ & Mudstone & \\
\hline 460 & $910.87-912.90$ & Mudstone-wackestone & Minor moldic porosity \\
\hline 461 & $912.90-913.20$ & Mudstone-wackestone & \\
\hline 462 & $913.20-913.80$ & Mudstone & \\
\hline 463 & $913.80-917.11$ & Packstone & Very porous; intraclasts (Core 97R) \\
\hline 464 & $917.11-917.75$ & Grainstone & \\
\hline $\begin{array}{l}465 \\
466\end{array}$ & & $\begin{array}{l}\text { Floatstone } \\
\text { Wackestone }\end{array}$ & $\begin{array}{l}\text { (Core 98R) } \\
\text { Very porous; limestone intraclasts; dissolution halos }\end{array}$ \\
\hline $\begin{array}{l}466 \\
467\end{array}$ & $\begin{array}{l}919.11-923.91 \\
923.91-924.53\end{array}$ & $\begin{array}{l}\text { Wackestone } \\
\text { Rudstone-grainstone }\end{array}$ & Very porous; limestone intraclasts; dissolution halos \\
\hline 468 & $924.53-928.43$ & Laminites & $\begin{array}{l}\text { Clay-rich algal laminites with packstone intervals; moldic porosity at } \\
\text { base of section }\end{array}$ \\
\hline 469 & $928.43-929.70$ & Grainstone & Intense dissolution $828.74-828.87$ \\
\hline 470 & $929.70-930.75$ & Packstone & Nodular texture \\
\hline 471 & $930.75-931.43$ & Grainstone & \\
\hline 472 & $931.43-931.95$ & Mudstone & Massive \\
\hline 473 & $931.95-932.40$ & Mudstone-wackestone & Small and large vugs; intraclasts \\
\hline 474 & $932.40-933.91$ & Packstone & Well-cemented interval 933.2 \\
\hline 475 & $933.91-934.29$ & Mudstone & Finely bedded; clay seams \\
\hline 476 & $935.30-937.02$ & Packstone & Moldic porosity \\
\hline 477 & $937.02-937.48$ & Laminites & \\
\hline 478 & $937.48-938.25$ & Mudstone & Small vugs $(<1 \mathrm{~cm})$ \\
\hline 479 & $938.25-940.47$ & Packstone & Large vugs $(>1 \mathrm{~cm})$ \\
\hline 480 & $940.47-941.56$ & Packstone & Upper portion well-cemented and less vugular \\
\hline 481 & $941.56-942.95$ & Mudstone-wackestone & \\
\hline 482 & $942.95-946.74$ & Wackestone-mudstone & (Cores 100R, 101R) \\
\hline 483 & $946.74-948.53$ & Mudstone-wackestone & \\
\hline 484 & $948.53-949.19$ & Laminites & \\
\hline 485 & 949.19-949.44 & Mudstone & Massive \\
\hline $\begin{array}{l}486 \\
487\end{array}$ & $949.44-949.93$ & Laminites & \\
\hline $\begin{array}{l}487 \\
488\end{array}$ & $\begin{array}{l}949.93-951.76 \\
951.76-952.19\end{array}$ & $\begin{array}{l}\text { Packstone } \\
\text { Mudstone }\end{array}$ & $\begin{array}{l}\text { Moldic porosity } \\
\text { Minor moldic porosity }\end{array}$ \\
\hline 489 & $952.19-952.92$ & Laminites & \\
\hline 490 & $952.92-953.81$ & Wackestone-mudstone & \\
\hline 491 & $953.81-955.48$ & Packstone-wackestone & Grades upward to wackestone-packstone; moldic porosity \\
\hline 492 & $955.48-962.77$ & Packstone & Grades upward to mudstone-wackestone \\
\hline 493 & $962.77-964.68$ & Laminites & \\
\hline 494 & $964.68-965.28$ & Mudstone & \\
\hline 495 & $965.28-968.20$ & Packstone & Very porous at base; grades upward to mudstone \\
\hline 496 & $968.20-968.46$ & Mudstone & \\
\hline 497 & $968.46-968.54$ & Packstone & Gl- \\
\hline 498 & $968.54-969.23$ & Wackestone & Clay seam at 968.92 (Core 103R) \\
\hline 499 & $969.23-972.15$ & Packstone & Grades upward to packstone; extensive dissolution (Cores 103R, 104R) \\
\hline
\end{tabular}


Table 2 (continued).

\begin{tabular}{|c|c|c|c|}
\hline $\begin{array}{c}\text { Bed } \\
\text { number }\end{array}$ & $\begin{array}{l}\text { Depth } \\
\text { range (mbsf) }\end{array}$ & Lithology & Description \\
\hline 500 & $972.15-976.88$ & Laminites & (Core 104R) \\
\hline 501 & $976.88-977.15$ & Wackestone & (Core 104R) \\
\hline 502 & $977.15-981.30$ & Packstone & Extensive dissolution at base of section \\
\hline 503 & $981.30-985.86$ & Wackestone-mudstone & $\begin{array}{l}\text { Minor moldic porosity; limestone intraclasts (Core 105R) 984-985 } \\
\text { interval predominantly mudstone }\end{array}$ \\
\hline 504 & $985.86-986.03$ & Laminites & \\
\hline 505 & $986.03-986.14$ & Mudstone-wackestone & \\
\hline 506 & $986.14-986.20$ & Laminites & \\
\hline 507 & $986.20-987.31$ & Mudstone-wackestone & Moldic porosity; intraclasts \\
\hline 508 & $987.31-988.88$ & Wackestone-mudstone & Mudstone interbeds \\
\hline 509 & $988.88-989.70$ & Packstone & Moldic porosity \\
\hline 510 & $989.70-990.77$ & Packstone & Intense dissolution \\
\hline 511 & $990.77-991.09$ & Wackestone-mudstone & \\
\hline 512 & $991.09-993.34$ & Wackestone-mudstone & Wackestone grading upward to mudstone; intraclasts at base \\
\hline 513 & $993.34-993.46$ & Mudstone & \\
\hline 514 & $993.46-996.55$ & Mudstone-wackestone & Intraclasts \\
\hline 515 & $996.55-998.77$ & Packstone & Numerous rounded intraclasts \\
\hline 516 & $998.77-999.37$ & Laminites & \\
\hline 517 & $999.37-999.56$ & Clay & Limestone intraclast \\
\hline 518 & $999.56-1000.85$ & Packstone & Porosity increases downsection \\
\hline 519 & $1000.85-1001.80$ & Wackestone-mudstone & \\
\hline 520 & $1001.80-1002.12$ & Laminites & \\
\hline 521 & $1002.12-1002.42$ & Mudstone & Massive \\
\hline 522 & $1002.42-1002.45$ & Clay & \\
\hline 523 & $1002.45-1002.60$ & Mudstone & \\
\hline 524 & $1002.60-1002.93$ & Wackestone & \\
\hline 525 & $1002.93-1003.27$ & Mudstone & \\
\hline 526 & $1003.27-1007.65$ & Packstone-wackestone & $\begin{array}{l}\text { Packstone grading upward to wackestone; moldic porosity; extensive } \\
\text { dissolution }\end{array}$ \\
\hline 527 & $1007.65-1009.38$ & Packstone-wackestone & $\begin{array}{l}\text { Packstone grading upward to wackestone; moldic porosity; extensive } \\
\text { dissolution }\end{array}$ \\
\hline 528 & $1009.38-1010.43$ & Laminites & \\
\hline 529 & $1010.43-1011.70$ & Wackestone & \\
\hline 530 & $1011.70-1012.07$ & Wackestone-grainstone & Interbedded wackestone and grainstone \\
\hline 531 & $1012.07-1013.52$ & Wackestone & Mudstone intraclasts; clay seam at 1012.07 \\
\hline 532 & $1013.52-1014.52$ & Grainstone-wackestone & Clay seam at 1014.5 \\
\hline 533 & $1014.52-1015.37$ & Wackestone & \\
\hline 534 & $1015.37-1015.52$ & Mudstone & Massive; low porosity \\
\hline 535 & $1015.52-1018.45$ & Packstone-wackestone & $\begin{array}{l}\text { Bottom } 0.4 \mathrm{~m} \text { is wackestone, grading upward to packstone; Mudstone } \\
\text { intraclasts (Core 108R) }\end{array}$ \\
\hline 536 & $1018.45-1022.68$ & Grainstone & $\begin{array}{l}\text { Coarse grainstones grading upward to fine grainstone; mudstone rip-up } \\
\text { clasts at base of section }\end{array}$ \\
\hline 537 & $1022.68-1023.86$ & Mudstone & Algal laminites and clay seams \\
\hline 538 & $1023.86-1025.20$ & Laminites-algal mat & Minor mudstone intervals \\
\hline 539 & $1025.20-1026.10$ & Laminites & \\
\hline 540 & $1026.10-1027.22$ & Mudstone & \\
\hline 541 & $1027.22-1032.82$ & Packstone & Moldic porosity; burrowed; intraclasts (Core 110R) \\
\hline 542 & $1032.82-1033.32$ & Packstone-algal mat & (Core $110 \mathrm{R})$ \\
\hline 543 & $1033.32-1034.74$ & Wackestone-algal mat & Moldic porosity (Core $110 \mathrm{R}$ ) \\
\hline 544 & $1034.74-1036.83$ & Laminites & Some wackestone; minor packstone at top of section (Core 110R) \\
\hline 545 & $1036.83-1039.60$ & Packstone-wackestone & Packstone grading upward to wackestone; moldic porosity; burrowed \\
\hline 546 & $1039.60-1042.08$ & Wackestone-packstone & $\begin{array}{l}\text { Packstone grading upward to wackestone-grainstone; moldic porosity; } \\
\text { intraclasts }\end{array}$ \\
\hline 547 & $1042.08-1042.78$ & Packstone-algal mat & \\
\hline 548 & $1042.78-1043.29$ & Laminites-algal mat & Graded bedding \\
\hline 549 & $1043.29-1044.54$ & Rudstone & \\
\hline 550 & $1044.54-1044.92$ & Wackestone-rudstone & \\
\hline 551 & $1044.92-1046.00$ & Mudstone & Some laminites; clay seam \\
\hline 552 & $1046.00-1051.83$ & Wackestone-mudstone & $\begin{array}{l}\text { Wackestone grading upward to wackestone-mudstone; minor packstone } \\
\text { (Core } 111 \mathrm{R} \text { ) }\end{array}$ \\
\hline 553 & $1051.83-1053.86$ & Wackestone & \\
\hline 554 & $1053.86-1054.83$ & Packstone & Moldic porosity \\
\hline 555 & $1054.83-1055.71$ & wackestone & Moldic porosity \\
\hline 556 & $1055.71-1056.01$ & Laminites & \\
\hline 557 & $1056.01-1060.16$ & Wackestone-mudstone & Wackestone grading upward to wackestone-mudstone \\
\hline 558 & $1060.16-1062.00$ & Grainstone & \\
\hline 559 & $1062.00-1062.25$ & Packstone & (Core 113R) \\
\hline 560 & $1062.25-1065.02$ & Wackestone & \\
\hline 561 & $1065.02-1066.11$ & wackestone & Well-cemented \\
\hline 562 & $1066.11-1066.65$ & Laminites & Clay seams \\
\hline 563 & $1066.65-1067.47$ & Clay seams & With laminites \\
\hline 564 & $1067.47-1069.96$ & Wackestone & \\
\hline 565 & $1069.96-1070.87$ & Laminites & \\
\hline 566 & $1070.87-1072.10$ & Packstone-wackestone & Packstone grading upward to wackestone \\
\hline 567 & $1072.10-1072.46$ & Laminites & \\
\hline 568 & $1072.46-1073.27$ & Wackestone & \\
\hline 569 & $1073.27-1074.76$ & Packstone & \\
\hline 570 & $1074.76-1075.80$ & Wackestone & Clay seams (Core 114R) \\
\hline 571 & $1075.80-1076.25$ & Packstone & (Core 114R) \\
\hline 572 & $1076.25-1077.06$ & Laminites & \\
\hline 573 & $1077.06-1078.14$ & Mudstone & Clay-rich laminites \\
\hline 574 & $1078.14-1081.22$ & Packstone & $\begin{array}{l}\text { Packstone grading upward to wackestone minor moldic porosity (Core } \\
115 \mathrm{R}) \text {; clay seams }\end{array}$ \\
\hline 575 & $1081.22-1082.22$ & Wackestone & Clay seams \\
\hline 576 & $1082.22-1087.32$ & Packstone & \\
\hline 577 & $1087.32-1088.69$ & Packstone-wackestone & \\
\hline 578 & $1088.69-1090.00$ & Laminites & \\
\hline
\end{tabular}


Table 2 (continued).

\begin{tabular}{|c|c|c|c|}
\hline $\begin{array}{c}\text { Bed } \\
\text { number }\end{array}$ & $\begin{array}{l}\text { Depth } \\
\text { range (mbsf) }\end{array}$ & Lithology & Description \\
\hline 579 & $1090.00-1096.04$ & Packstone-wackestone & $\begin{array}{l}\text { Very coarse and vugular packstone at base, grading upward to } \\
\text { wackestone }\end{array}$ \\
\hline 580 & $1096.04-1096.80$ & Laminites & (Core $116 R)$ \\
\hline 581 & $1096.80-1097.20$ & Wackestone & \\
\hline 582 & $1097.20-1097.56$ & Laminites & \\
\hline 583 & $1097.56-1097.67$ & Wackestone & \\
\hline 584 & $1097.67-1098.07$ & $\begin{array}{l}\text { Algal mat } \\
\text { Wackestone }\end{array}$ & With laminites \\
\hline $\begin{array}{l}585 \\
586\end{array}$ & $\begin{array}{l}1098.07-1099.87 \\
109987-110034\end{array}$ & $\begin{array}{l}\text { Wackestone } \\
\text { Wackestone }\end{array}$ & Very well-cemented \\
\hline $\begin{array}{l}586 \\
587\end{array}$ & $\begin{array}{l}1099.87-1100.34 \\
1100.34-1100.67\end{array}$ & $\begin{array}{l}\text { Wackestone } \\
\text { Laminites }\end{array}$ & Clay seam \\
\hline 588 & $1100.67-1102.00$ & Laminites & \\
\hline 589 & $1102.00-1105.51$ & Packstone & (Core 117R) \\
\hline 590 & $1105.51-1107.30$ & Laminites & Clay seams (Core 117R) \\
\hline 591 & $1107.30-1107.63$ & Wackestone & \\
\hline 592 & $1107.63-1107.84$ & Laminites & Clay seam \\
\hline 593 & $1107.84-1108.40$ & Wackestone & \\
\hline 594 & $1108.40-1109.02$ & $\begin{array}{l}\text { Laminites } \\
\text { Packstone }\end{array}$ & Packstone interbedded with clay; minor moldic porosity \\
\hline 595 & $1109.02-1109.58$ & $\begin{array}{l}\text { Packstone } \\
\text { Packstone }\end{array}$ & $\begin{array}{l}\text { Packstone interbedded with clay; minor moldic porosity } \\
\text { Moldic porosity }\end{array}$ \\
\hline $\begin{array}{l}596 \\
597\end{array}$ & $\begin{array}{l}1109.58-1110.90 \\
1110.90-1111.45\end{array}$ & $\begin{array}{l}\text { Packstone } \\
\text { Wackestone }\end{array}$ & \\
\hline 598 & $1111.45-1112.32$ & Packstone & \\
\hline 599 & $1112.32-1113.40$ & Laminites & Clay-rich intervals \\
\hline 600 & $1113.40-1114.50$ & $\begin{array}{l}\text { Packstone } \\
\text { Laminites }\end{array}$ & \\
\hline 601 & $1114.50-1115.46$ & $\begin{array}{l}\text { Laminites } \\
\text { Wackestone-packstone }\end{array}$ & \\
\hline 602 & $1115.46-1117.09$ & $\begin{array}{l}\text { Wackestone-packstone } \\
\text { Laminites }\end{array}$ & Wackestone grading upward to packstone \\
\hline 603 & $1117.09-1118.02$ & $\begin{array}{l}\text { Laminites } \\
\text { Packstone }\end{array}$ & \\
\hline 604 & $1118.02-1118.85$ & $\begin{array}{l}\text { Packstone } \\
\text { Laminites }\end{array}$ & (Core 119R) \\
\hline 605 & $1118.85-1119.73$ & $\begin{array}{l}\text { Laminites } \\
\text { Grainstone }\end{array}$ & \\
\hline 606 & $1119.73-1121.35$ & $\begin{array}{l}\text { Grainstone } \\
\text { Rudstone }\end{array}$ & \\
\hline $\begin{array}{l}607 \\
608\end{array}$ & $\begin{array}{l}1121.35-1122.55 \\
1122.55-1124.74\end{array}$ & $\begin{array}{l}\text { Rudstone } \\
\text { Mudstone-grainstone }\end{array}$ & Clay-rich intervals \\
\hline 609 & $1124.74-1126.80$ & Grainstone & Intraclast-rich interval (1125.1-1125.7) \\
\hline 610 & $1126.80-1127.30$ & Packstone-grainstone & \\
\hline 611 & $1127.30-1128.20$ & Laminites & \\
\hline 612 & $1128.20-1129.18$ & Grainstone & Well-cemented at base of section; moldic porosity \\
\hline 613 & $1129.18-1129.58$ & Mudstone & Massive \\
\hline 614 & $1129.58-1130.22$ & Packstone-grainstone & (Core 120R) \\
\hline 615 & $1130.22-1131.03$ & $\begin{array}{l}\text { Grainstone } \\
\text { Laminites }\end{array}$ & \\
\hline 616 & $1131.03-1131.61$ & $\begin{array}{l}\text { Laminites } \\
\text { Packstone }\end{array}$ & Minor moldic porosity \\
\hline $\begin{array}{l}617 \\
618\end{array}$ & $\begin{array}{l}1131.61-1133.01 \\
1133.01-1133.43\end{array}$ & $\begin{array}{l}\text { Packstone } \\
\text { Mudstone }\end{array}$ & With algal mat \\
\hline $\begin{array}{l}618 \\
619\end{array}$ & $1133.43-1134.13$ & Wackestone & \\
\hline 620 & $1134.13-1135.07$ & Grainstone & \\
\hline 621 & $1135.07-1136.54$ & Packstone-grainstone & Moldic porosity \\
\hline 622 & $1136.54-1136.94$ & Wackestone & \\
\hline 623 & $1136.94-1137.53$ & Laminites & \\
\hline 624 & $1137.53-1137.93$ & Wackestone & Highly resistive; very well cemented or dolomitized \\
\hline 625 & $1137.93-1140.05$ & Wackestone-grainstone & Wackestone and grainstone interbeds; intraclasts (dolomite?) \\
\hline 626 & $1140.05-1142.01$ & Packstone & $\begin{array}{l}\text { Intergranular porosity increases upsection; numerous intraclasts in upper } \\
\text { half }\end{array}$ \\
\hline 627 & $1142.01-1142.54$ & Wackestone & Rounded intraclasts; large vugs \\
\hline 628 & $1142.54-1143.83$ & Laminites & Clay seam \\
\hline 629 & $1143.83-1144.55$ & Grainstone-wackestone & \\
\hline 630 & $1144.55-1146.30$ & Packstone-grainstone & Thin, well-cemented intervals \\
\hline 631 & $1146.30-1148.05$ & Grainstone-wackestone & \\
\hline $\begin{array}{l}632 \\
633\end{array}$ & $\begin{array}{l}1148.05-1151.05 \\
1151.05-1152.00\end{array}$ & $\begin{array}{l}\text { Grainstone-packstone } \\
\text { Packstone }\end{array}$ & $\begin{array}{l}\text { Grainstone grading upward to packstone } \\
\text { Moldic porosity }\end{array}$ \\
\hline 634 & $1152.00-1152.50$ & Mudstone & Small vugs \\
\hline 635 & $1152.50-1154.20$ & Packstone & Moldic porosity; dolomitized \\
\hline 636 & $1154.20-1156.60$ & Grainstone & Moldic porosity \\
\hline 637 & $1156.60-1157.39$ & Dolomitized packstone & \\
\hline 638 & $1157.39-1157.70$ & Mudstone & \\
\hline 639 & $1157.70-1159.04$ & Laminites & \\
\hline 640 & $1159.04-1162.32$ & $\begin{array}{l}\text { Packstone } \\
\text { Mudstone }\end{array}$ & \\
\hline 641 & $1162.32-1163.06$ & $\begin{array}{l}\text { Mudstone } \\
\text { Grainstone }\end{array}$ & \\
\hline $\begin{array}{l}642 \\
643\end{array}$ & $\begin{array}{l}1163.06-1163.44 \\
1163.44-1163.77\end{array}$ & $\begin{array}{l}\text { Grainstone } \\
\text { Mudstone }\end{array}$ & \\
\hline 644 & $1163.77-1165.04$ & Grainstone & Vertical fracture \\
\hline 645 & $1165.04-1165.57$ & Dolomite & \\
\hline 646 & $1165.57-1166.15$ & Grainstone & \\
\hline 647 & $1166.15-1166.75$ & Packstone & Partially dolomitized; clay seams \\
\hline 648 & $1166.75-1167.41$ & Wackestone & \\
\hline 649 & $1167.41-1168.60$ & Grainstone & \\
\hline 650 & $1168.60-1168.66$ & $\begin{array}{l}\text { Clay } \\
\text { Laminites }\end{array}$ & \\
\hline 651 & $1168.66-1169.28$ & $\begin{array}{l}\text { Laminites } \\
\text { Packstone-grainstone }\end{array}$ & Packstone grading upward to grainstone; dolomitized (Core 124R) \\
\hline $\begin{array}{l}652 \\
653\end{array}$ & $\begin{array}{l}1169.28-1171.43 \\
1171.43-1172.30\end{array}$ & $\begin{array}{l}\text { Packstone-grainstone } \\
\text { Grainstone }\end{array}$ & $\begin{array}{l}\text { Packstone grading upward to grainstone, doromituzed (Core } 124 \mathrm{~K} \text { ) } \\
\text { (Dolomite?) nodules }\end{array}$ \\
\hline $\begin{array}{l}653 \\
654\end{array}$ & $\begin{array}{l}1171.43-1172.30 \\
1172.30-1173.45\end{array}$ & $\begin{array}{l}\text { Grainstone } \\
\text { Wackestone-packstone }\end{array}$ & Wackestone grading upward to packstone \\
\hline 655 & $1173.45-1174.16$ & Grainstone & Wavy bedding; clay seam \\
\hline 656 & $1174.16-1176.40$ & Dolomitized packstone & \\
\hline 657 & $1176.40-1178.67$ & Packstone-grainstone & Dolomite nodules \\
\hline 658 & $1178.67-1184.02$ & Grainstone-laminites & $\begin{array}{l}\text { Laminites interbedded with grainstones and clay seams; minor moldic } \\
\text { porosity }\end{array}$ \\
\hline 659 & $1184.02-1185.20$ & Mudstone & Massive \\
\hline 660 & $1185.20-1186.63$ & Grainstone & Minor wackestone at base of section (Core 126R) \\
\hline 661 & $1186.63-1187.40$ & Grainstone & Clay seams \\
\hline 662 & $1187.40-1187.63$ & Packstone & \\
\hline 663 & $1187.63-1188.07$ & Wackestone & \\
\hline
\end{tabular}


Table 2 (continued).

\begin{tabular}{|c|c|c|c|}
\hline $\begin{array}{c}\text { Bed } \\
\text { number }\end{array}$ & $\begin{array}{l}\text { Depth } \\
\text { range (mbsf) }\end{array}$ & Lithology & Description \\
\hline 664 & $1188.07-1193.52$ & Grainstone & Clay seams; minor packstone intervals; minor moldic porosity \\
\hline 665 & $1193.52-1195.41$ & Wackestone-mudstone & Porosity decreases upsection \\
\hline 666 & $1195.41-1197.55$ & Grainstones & Clay seams; minor packstone \\
\hline 667 & $1197.55-1198.48$ & Wackestone & \\
\hline 668 & $1198.48-1203.31$ & Wackestone-grainstone & Clay seams; intraclasts (Core 127R) \\
\hline 669 & $1203.31-1204.40$ & Grainstone-packstone & Laminite interbeds (Core 128R) \\
\hline 670 & $1204.40-1206.02$ & Dolomitized packstone & \\
\hline 671 & $1206.02-1207.71$ & Grainstone & Clay seams; minor moldic porosity; well-cemented \\
\hline 672 & $1207.71-1208.42$ & Wackestone-grainstone & Partially dolomitized; minor moldic porosity \\
\hline 673 & $1208.42-1209.06$ & Grainstone & Partially dolomitized \\
\hline 674 & $1209.06-1212.11$ & Wackestone & Dolomitized in lower portion \\
\hline 675 & $1212.11-1212.80$ & Grainstone & \\
\hline 676 & $1212.80-1214.47$ & Dolomite & Upper and lower portions retain some grainstone texture \\
\hline 677 & $1214.47-1216.42$ & Wackestone-grainstone & $\begin{array}{l}\text { Clay seams; minor moldic porosity; lower } 40 \mathrm{~cm} \text { very well-cemented } \\
\text { (Core } 129 \mathrm{R} \text { ) }\end{array}$ \\
\hline 678 & $1216.42-1217.36$ & Dolomite & Some grainstone texture remains; clay seams \\
\hline 679 & $1217.36-1219.90$ & Grainstone-packstone & Clay seams; patchy dolomitization \\
\hline 680 & $1219.90-1221.16$ & Packstone & Dolomitized at base \\
\hline 681 & $1221.16-1221.67$ & Packstone-grainstone & \\
\hline 682 & $1221.67-1223.60$ & Grainstone & Dolomite burrow fillings \\
\hline 683 & $1223.60-1225.02$ & Mudstone & \\
\hline 684 & $1225.02-1225.88$ & Packstone-grainstone & Dolomitized \\
\hline 685 & $1225.88-1226.87$ & Packstone-rudstone & \\
\hline 686 & $1226.87-1228.67$ & Grainstone & Clay seams, laminite interbeds \\
\hline 687 & $1228.67-1229.47$ & Packstone & Moldic porosity \\
\hline 688 & $1229.47-1230.78$ & Wackestone & Clay seams, laminite interbeds \\
\hline 689 & $1230.78-1236.48$ & Grainstone & $\begin{array}{l}\text { Laminated claystones and clay seams; minor moldic porosity; } \\
\text { dolomitized }\end{array}$ \\
\hline 690 & $1236.48-1236.86$ & Wackestone & Patchy dolomitization (Core 131R) \\
\hline 691 & $1236.86-1237.19$ & Laminites & Laminated algal mat and clayseams \\
\hline 692 & $1237.19-1238.69$ & Grainstone-packstone & Clay seam \\
\hline 693 & $1238.69-1241.69$ & Packstone-grainstone & Clay laminites \\
\hline 694 & $1241.69-1242.96$ & Wackestone-grainstone & \\
\hline 695 & $1242.96-1244.21$ & Dolomite & Some grainstone texture remains; clay seam at top \\
\hline 696 & $1244.21-1244.54$ & Wackestone & (Core 132R) \\
\hline 697 & $1244.54-1246.20$ & Grainstone & Dolomitized; moldic porosity; intraclasts \\
\hline 698 & $1246.20-1247.78$ & Grainstone & Slightly dolomitized \\
\hline 699 & $1247.78-1247.96$ & Packstone & \\
\hline 700 & $1247.96-1250.50$ & Grainstone & Clay seams \\
\hline 701 & $1250.50-1252.40$ & Grainstone & Small-scale $(<1 \mathrm{~cm})$ moldic porosity \\
\hline 702 & $1252.40-1253.44$ & Grainstone & Dolomitized \\
\hline 703 & $1253.44-1254.12$ & Packstone & Patchy dolomitization \\
\hline 704 & $1254.12-1258.27$ & Dolomite & Rudstone texture (Core 133R) \\
\hline 705 & $1258.27-1265.00$ & Dolomite & $\begin{array}{l}\text { Rudstone texture; numerous intervals of compacted limestone with } \\
\text { stylolites (Core 134R) }\end{array}$ \\
\hline 706 & $1265.00-1269.24$ & Wackestone & Dolomitized; vugular; largest vugs $4-5 \mathrm{~cm}$ \\
\hline 707 & $1269.24-1269.80$ & Dolomite & Moldic porosity; some grainstone texture remains \\
\hline 708 & $1269.80-1271.11$ & Wackestone & Stylolites \\
\hline 709 & $1271.11-1272.00$ & Dolomite & Some grainstone texture remains \\
\hline 710 & $1272.00-1273.29$ & Packstone-grainstone & Dolomitized; stylolites \\
\hline 711 & $1273.29-1274.50$ & Dolomite & Rudstone texture (Core 135R) \\
\hline 712 & $1274.50-1277.20$ & Packstone & Patchy dolomitization; stylolites \\
\hline 713 & $1277.20-1277.89$ & Dolomite & Very porous \\
\hline 714 & $1277.89-1280.60$ & Dolomite & Some grainstone texture remains \\
\hline 715 & $1280.60-1286.56$ & Wackestone & Numerous intraclasts; dolomite infillings \\
\hline 716 & $1286.56-1288.08$ & Dolomite & Relict grainstone texture \\
\hline 717 & $1288.08-1291.09$ & Dolomite & Wackestone-rudstone texture; vugular \\
\hline 718 & $1291.09-1292.60$ & Dolomite & Rudstone texture \\
\hline 719 & $1292.60-1293.72$ & Packstone & Patchy dolomitization \\
\hline 720 & $1293.72-1295.70$ & Dolomite & Rudstone texture \\
\hline 721 & $1295.70-1297.78$ & Wackestone & Partially dolomitized \\
\hline 722 & $1297.78-1303.15$ & Wackestone & Minor dolomitization within two vugular intervals \\
\hline 723 & $1303.15-1303.72$ & Wackestone & Partially dolomitized \\
\hline 724 & $1303.72-1307.12$ & Grainstone & Clay seams; $1304.0-1304.6$ dolomitized (Core 138R) \\
\hline 725 & $1307.12-1308.11$ & Dolomite & \\
\hline 726 & $1308.11-1308.68$ & Grainstone & \\
\hline 727 & $1308.68-1310.16$ & Dolomite & Vugular \\
\hline 728 & $1310.16-1311.80$ & Packstone-grainstone & Dolomitized; clay seams \\
\hline 729 & $1311.80-1314.81$ & Grainstone & Thinly bedded (Core 139R) \\
\hline 730 & $1314.81-1315.24$ & Dolomite & \\
\hline 731 & $1315.24-1315.85$ & Wackestone-grainstone & \\
\hline 732 & $1315.85-1318.73$ & Dolomite & Minor intraclasts; vugular (Core 140R) \\
\hline 733 & $1318.73-1320.85$ & Grainstone & Partially dolomitized; numerous intraclasts; vugular (Core 140R) \\
\hline 744 & $1320.85-1330.49$ & Grainstone & Minor packstone; partially dolomitized \\
\hline 745 & $1330.49-1331.93$ & Dolomite & Relict grainstone texture (Core 141R) \\
\hline 746 & $1331.93-1337.83$ & Packstone & Dolomitized; highly vugular; highly variable porosity \\
\hline 747 & $1337.83-1341.38$ & Dolomite & Rudstone texture \\
\hline 748 & $1341.38-1343.59$ & Wackestone-grainstone & Partially dolomitized \\
\hline 749 & $1343.59-1345.64$ & Grainstone & Minor dolomitization; nodules of dolomite \\
\hline 750 & $1345.64-1345.80$ & Laminites-clay & \\
\hline 751 & $1345.80-1346.80$ & Grainstone & \\
\hline 752 & $1346.80-1346.90$ & Clay & \\
\hline 753 & $1346.90-1350.25$ & Grainstone & Well-sorted; variable dolomitization; numerous dolomite nodules \\
\hline 754 & $1350.25-1353.67$ & Dolomite & Vugular, relict grainstone texture at base of section \\
\hline 755 & $1353.67-1354.60$ & Grainstone & Partially dolomitized (Core 143R) \\
\hline 756 & $1354.60-1355.11$ & Dolomite & Rudstone texture \\
\hline 757 & $1355.11-1357.18$ & Grainstone-wackestone & \\
\hline 758 & $1357.18-1358.90$ & Grainstone & Dolomitized; minor dolomite nodules \\
\hline
\end{tabular}


Table 2 (continued).

\begin{tabular}{|c|c|c|c|}
\hline $\begin{array}{c}\text { Bed } \\
\text { number }\end{array}$ & $\begin{array}{c}\text { Depth } \\
\text { range (mbsf) }\end{array}$ & Lithology & Description \\
\hline 759 & $1358.90-1359.25$ & Wackestone & \\
\hline 760 & $1359.25-1359.59$ & Laminites-clay & \\
\hline 761 & $1359.59-1361.26$ & Packstone & \\
\hline 762 & $1361.26-1361.35$ & Clay & \\
\hline 763 & $1361.35-1362.77$ & Wackestone & Minor moldic porosity; intraclasts \\
\hline 764 & $1362.77-1363.20$ & Grainstone & Well-sorted \\
\hline 765 & $1363.20-1363.50$ & Clay-laminites & \\
\hline 766 & $1363.50-1364.10$ & Grainstone & Well-sorted (Core 144R) \\
\hline 767 & $1364.10-1364.40$ & Marl & Clay seam at base of section (Core 144R) \\
\hline 768 & $1364.40-1366.09$ & Grainstone & (Core 144R) \\
\hline 769 & $1366.09-1367.18$ & Wackestone & Dolomitization increases downsection \\
\hline 770 & $1367.18-1372.00$ & Dolomite & Clay seam 1368.14 \\
\hline 771 & $1372.00-1373.00$ & Packstone-grainstone & \\
\hline 772 & $1373.00-1373.60$ & Dolomite & Relict grainstone texture \\
\hline 773 & $1373.60-1374.17$ & Packstone-grainstone & \\
\hline 774 & $1374.17-1375.04$ & Dolomite & Rudstone texture \\
\hline 775 & $1375.04-1376.12$ & Mudstone & Finely laminated clay-mudstone intervals \\
\hline 776 & $1376.12-1376.22$ & Clay & \\
\hline 777 & $1376.22-1379.07$ & Packstone-grainstone & Clay seam 1377.65 \\
\hline 778 & $1379.07-1379.88$ & Dolomite & Stylolites \\
\hline 779 & $1379.88-1380.22$ & Packstone-grainstone & \\
\hline 780 & $1380.22-1381.47$ & Mudstone & Prominent stylolites (Core 146R) \\
\hline 781 & $1381.47-1381.54$ & Clay & \\
\hline 782 & $1381.54-1384.96$ & Mudstone-grainstone & Stylolites \\
\hline 783 & $1384.96-1386.49$ & Grainstone & Numerous clay seams \\
\hline 784 & $1386.49-1388.60$ & Packstone-grainstone & Dolomitized; rudstone texture at base of section \\
\hline 785 & $1388.60-1393.66$ & Grainstone & $\begin{array}{l}\text { Clays and laminite plentiful in interval 1392.8-1393.66; minor packstone } \\
\text { interval 1391.2-1391.6 }\end{array}$ \\
\hline 786 & $1393.66-1394.90$ & Wackestone-mudstone & Flaser bedding at base of section (Core 147R) \\
\hline 787 & $1394.90-1397.72$ & Grainstone-mudstone & Uniform porosity; numerous clay seams (Core 147R) \\
\hline 788 & $1397.72-1398.84$ & Dolomite & Rudstone texture \\
\hline 789 & $1398.84-1399.36$ & Packstone & \\
\hline 790 & $1399.36-1400.31$ & Wackestone & Minor clay seams \\
\hline 791 & $1400.31-1403.13$ & Mudstone-grainstone & Numerous clay seams; anastomosing clay seams at 1401.2 \\
\hline 792 & $1403.13-1404.12$ & Mudstone-grainstone & Pebbles at base of section \\
\hline 793 & $1404.12-1406.04$ & Grainstone & Thinly bedded; upward-fining; clay seams \\
\hline 794 & $1406.04-1408.00$ & Packstone-rudstone & \\
\hline 795 & $1408.00-1409.80$ & Packstone-grainstone & \\
\hline 796 & $1409.80-1410.40$ & Grainstone & Well-sorted (Core 149R) \\
\hline 797 & $1410.40-1420.16$ & Grainstone & Intervals of clay and mudstone; moldic porosity; variable porosity \\
\hline 798 & $1420.16-1423.00$ & Grainstone & $\begin{array}{l}\text { Well-sorted; moldic porosity increases downsection; intraclasts at base of } \\
\text { section (Core 150R) }\end{array}$ \\
\hline 799 & $1423.00-1427.83$ & Grainstone & $\begin{array}{l}\text { Variable porosity and grain size; minor clay seams; intraclasts (Core } \\
\text { 150R) }\end{array}$ \\
\hline 800 & $1427.83-1429.34$ & Grainstone & Well-sorted \\
\hline 801 & $1429.34-1441.20$ & Grainstone & $\begin{array}{l}\text { Variable porosity and grain size; clay seams and intraclasts more } \\
\text { prominent downsection }\end{array}$ \\
\hline 802 & $1441.20-1441.84$ & Grainstone & Well-sorted (Core 152R) \\
\hline 803 & $1441.84-1443.90$ & Grainstone & Dolomitized (Core 152R) \\
\hline 804 & $1443.90-1444.80$ & Grainstone & Dolomitized; clay seams; intraclast-rich intervals (Core 152R) \\
\hline 805 & $1444.80-1447.00$ & Grainstone & Partially dolomitized (Core 153R) \\
\hline 806 & $1447.00-1449.14$ & Grainstone & Thinly bedded (Core 153R) \\
\hline 807 & $1449.14-1450.18$ & Grainstone & Clay seams (Core 153R) \\
\hline 808 & $1450.18-1454.28$ & Grainstone & Dolomitized; dolomite infillings \\
\hline 809 & $1454.28-1454.66$ & Dolomite & Rudstone texture \\
\hline 810 & $1454.66-1456.50$ & Dolomite & Relict grainstone texture \\
\hline 811 & $1456.50-1475.85$ & Dolomite & Rudstone texture \\
\hline 812 & $1475.85-1458.60$ & Dolomite & Packstone-rudstone texture \\
\hline 813 & $1458.60-1461.42$ & Dolomite & Vugular \\
\hline 814 & $1461.42-1463.80$ & Packstone & Minor wackestone \\
\hline 815 & $1463.80-1464.68$ & Dolomite & Vugular \\
\hline 816 & $1464.68-1465.27$ & Packstone & \\
\hline 817 & $1465.27-1465.76$ & Mudstone & \\
\hline 818 & $1465.76-1467.00$ & Dolomite & Vugular \\
\hline 819 & $1467.00-1473.65$ & Dolomitized grainstone & Degree of dolomitization highly variable \\
\hline 820 & $1473.65-1480.91$ & Grainstone-rudstone & Moldic porosity; dolomite-filled burrows (1475.4); minor stylolites \\
\hline 821 & $1480.91-1482.66$ & Dolomite & Rudstone texture \\
\hline 822 & $1482.66-1505.88$ & Grainstone & Variable dolomitization; stylolites; rare clay seams \\
\hline 823 & $1505.88-1508.20$ & Grainstone-mudstone & Well-cemented; moldic porosity at 1506.4 \\
\hline 824 & $1508.20-1512.96$ & Dolomite & Relict texture similar to bed 823 \\
\hline 825 & $1512.96-1514.86$ & Wackestone & Minor moldic porosity \\
\hline 826 & $1514.86-1520.43$ & Dolomite & $\begin{array}{l}\text { Rudstone-grainstone texture; stylolites; dolomitization increases } \\
\text { downsection }\end{array}$ \\
\hline 827 & $1520.43-1521.38$ & Dolomite & Extremely porous \\
\hline 828 & $1521.38-1522.02$ & Wackestone & Well-cemented; moldic porosity \\
\hline 829 & $1522.02-1522.58$ & Clay-laminites & \\
\hline 830 & $1522.58-1525.82$ & Packstone-wackestone & Patchy dolomitization; moldic porosity \\
\hline 831 & $1525.82-1527.64$ & Grainstone & Dolomitized \\
\hline 832 & $1527.64-1529.55$ & Dolomite & Very porous \\
\hline 833 & $1529.55-1534.35$ & Dolomite & Relict grainstone texture intraclasts; well-cemented interval (1531.6) \\
\hline 834 & $1534.35-1534.80$ & Mudstone(?) & Well-cemented; stylolites \\
\hline 835 & $1534.80-1537.60$ & Dolomite & Rudstone texture \\
\hline 836 & $1537.60-1541.19$ & Grainstone & $\begin{array}{l}\text { Dolomitized; altemating well-cemented and porous intervals; porous } \\
\text { intervals show intergranular and vugular porosities }\end{array}$ \\
\hline 837 & $1541.19-1545.03$ & Dolomite & $\begin{array}{l}\text { Rudstone texture; vertical fluid escape channels; large cavity } 1542.4 \\
1542.8\end{array}$ \\
\hline 838 & $1545.03-1557.20$ & Dolomite & Relict grainstone texture \\
\hline 839 & $1557.20-1560.00$ & Mudstone & Dispersed organic matter \\
\hline
\end{tabular}


Table 2 (continued).

\begin{tabular}{|c|c|c|c|}
\hline $\begin{array}{c}\text { Bed } \\
\text { number }\end{array}$ & $\begin{array}{l}\text { Depth } \\
\text { range (mbsf) }\end{array}$ & Lithology & Description \\
\hline & $1560.00-1565.00$ & Data section missing & \\
\hline 840 & $1565.00-1569.58$ & Dolomite & Rudstone texture \\
\hline 841 & $1569.58-1572.65$ & Grainstone & Dolomitized; intervals of moldic porosity; stylolites \\
\hline 842 & $1572.65-1574.79$ & Wackestone & \\
\hline 843 & $1574.79-1578.44$ & Grainstone & Dolomitized; high intergranular and vugular porosities \\
\hline 844 & $1578.44-1582.50$ & Grainstone & Slightly dolomitized; mudstone intervals and clay seams \\
\hline 845 & $1582.50-1586.40$ & Grainstone & $\begin{array}{l}\text { Dolomitized; high intergranular porosity; clay seams; anastomosing clay } \\
\text { seams } 1585.0\end{array}$ \\
\hline 846 & $1586.40-1588.00$ & Grainstone & Dolomitized \\
\hline 847 & $1588.00-1589.76$ & Dolomite & Rudstone texture \\
\hline 848 & $1589.76-1590.60$ & Dolomite & Packstone-rudstone texture \\
\hline 849 & $1590.60-1591.95$ & Mudstone & \\
\hline 850 & $1591.95-1596.03$ & Mudstone-grainstone & \\
\hline 851 & $1596.03-1597.04$ & Mudstone & Stylolites \\
\hline 852 & $1597.04-1597.93$ & Dolomite & Rudstone texture \\
\hline 853 & $1597.93-1599.20$ & Mudstone & Stylolites \\
\hline 854 & $1599.20-1623.80$ & Mudstone-grainstone & $\begin{array}{l}\text { Mudstone with stylolites; grainstones highly variable porosity; clay } \\
\text { intervals }(1600.0,1601.8,1603.0) \text {; intraclasts } 1610.7-1612.4 \text {; } \\
\text { dolomitization increases downsection }\end{array}$ \\
\hline
\end{tabular}

\section{Legend}

Boundstone/Framestone

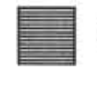

Grainstone

Wackestone

Wackestone-packstone

Packstone-wackestone

Wackestone-mudstone Mudstone-wackestone

Wackestone-rudstone

Rudstone-wackestone

Wackestone-grainstone Grainstone-wackestone

Packstone

Rudstone

Mudstone
Grainstone-rudstone Rudstone-grainstone

Packstone-grainstone Grainstone-packstone

Packstone-rudstone Rudstone-packstone

Mudstone-grainstone Grainstone-mudstone

Clay Seams

Marly limestone

Algal mat/laminites

Dolomite

Figure 8. Legend of patterns used to designate lithologies in Figures 9 and 10.

able width are not visible in the FMS images above about $657 \mathrm{mbsf}$; however, thin (below resolution) clay seams may have caused the stratification observed in the mudstones and wackestones-mudstones beginning at $603 \mathrm{mbsf}$. Concentrations of clay seams appear to be spaced at 20- to 50-cm intervals in the recovered core, but are spaced farther apart in the FMS images; again, probably because some thicknesses are below the resolution of the FMS tool. Clay seams may be the result of autocyclic processes, such as changing influx paths for terrigenous material or allocyclic fluctuation of sea level, rainfall intensity, carbonate production, or some combination of these (e.g., James, 1989).

Although the depth interval from 600 to 835.5 mbsf also consists of sequences of packstone, wackestone, and mudstone, the relatively greater abundance of mudstones, clay seams, and marls indicates a more restricted lagoonal setting. Grainstone intervals are rare and together with occurrences of rare planktonic species in the core material are probably the result of occasional washover.

In summary, the sedimentary record of Allison Guyot shows small-scale, shallow-water carbonate sequences of (late) Albian age. Facies imply a clay-, organic-, and pyrite-rich, marshlike environment with a nearby volcanic landmass in the lower part of the hole, and restricted, lagoonal settings in its upper part, similar (but not identical) to the Albian section at Hole 866A. The absence of clays above 600 mbsf suggests an expanding lagoon and submergence of the volcanic edifice.

\section{SUMMARY OF LOG-CORE INTEGRATION FOR HOLE 866A}

The depth interval from 206 to 466 mbsf consists of sediments derived from a restricted, shallow, subtidal inner shelf. From 206 to $346 \mathrm{mbsf}$, rudstones and wackestone-packstone sequences are common, with minor mudstone and grainstone. Extensive dissolution is evident at the base of this pile, indicating possible emergence and erosion of the platform. The mudstone-rudstone-wackestone facies common throughout the interval from 346 to 351 mbsf probably indicate a upward-deepening transition. Wackestones, packstones, and especially mudstones are common throughout the depth interval from 351 to $449 \mathrm{mbsf}$. Another upward-deepening transition is indicated by the packstones and marls that occur within the depth interval from 449 to 466 mbsf.

Tidal flats typified the depositional environment of sediments from the depth interval from 466 to 692 mbsf. The major lithologies consist of wackestone-packstone alternations with scattered abundant grainstones. Several distinct intervals can be distinguished on the basis of the presence or absence of grainstone or mudstone: from 466 to 479 mbsf, wackestone-packstone, grainstones abundant (slight progradation); 479 to 528 mbsf, wackestone-packstone, no mudstones; 528 to 543 mbsf, wackestone-packstone, grainstones abundant (slight progradation); 543 to 587 mbsf, wackestone-packstone, some grainstone and rudstone (period of maximum flooding; aggradation); 587 to 648 mbsf, wackestone-packstone, grainstones abundant (slight progradation); clay seams at 587 to $590 \mathrm{mbsf}$; 648 to $676 \mathrm{mbsf}$, wackestonemudstone and laminites (inner platform facies; aggradation); from 676 to $692 \mathrm{mbsf}$ is a transition facies, wackestone-mudstone, rudstone, grainstone (possibly a late highstand stage).

Deepening occurs upward over the interval from 692 to $798 \mathrm{mbsf}$. The grainstones recovered within the interval from 692 to $791 \mathrm{mbsf}$ reflect a foreshore environment during a highstand stage. The basal contact with underlying well-cemented mudstone is sharp. Slight backstepping is indicated by the mudstones of from 791 to $798 \mathrm{mbsf}$. 
Table 3. Bed thickness statistics for Holes 865A and 866A.

\begin{tabular}{|c|c|c|c|c|c|c|c|c|c|c|c|}
\hline \multicolumn{12}{|c|}{ Hole $865 \mathrm{~A}$} \\
\hline & Wackestone & Packstone & $\begin{array}{c}\text { Packstone } \\
\text {-wackestone }\end{array}$ & Mudstone & $\begin{array}{l}\text { Mudstone- } \\
\text { wackestone }\end{array}$ & Grainstone & Rudstone & Clay & Marl & Laminite & \\
\hline Min. & 0.09 & 0.08 & 0.34 & 0.09 & 0.32 & 0.13 & $* * * *$ & 0.05 & 0.43 & $* * * *$ & \\
\hline Max. & 5.55 & 35.98 & 8.73 & 4.26 & 16.08 & 7.74 & $* * * *$ & 1.05 & 2.76 & $* * * *$ & \\
\hline Sum & 22.56 & 349.01 & 59.37 & 15.26 & 198.88 & 22.18 & $* * * *$ & 2.13 & 11.23 & $* * * *$ & \\
\hline No. & 19 & 92 & 26 & 22 & 87 & 10 & $* * * *$ & 7 & 7 & $* * * *$ & \\
\hline Mean & 1.19 & 3.79 & 2.28 & 0.69 & 2.29 & 2.22 & $* * * *$ & 0.30 & 1.60 & $* * * *$ & \\
\hline$\%$ & 3 & 51 & 8 & 2 & 29 & 3 & $* * * *$ & $<1$ & 2 & $* * * *$ & \\
\hline \multicolumn{12}{|c|}{ Hole 866A } \\
\hline & Wackestone & Packstone & $\begin{array}{l}\text { Packstone } \\
\text {-wackestone }\end{array}$ & Mudstone & $\begin{array}{l}\text { Mudstone- } \\
\text { wackestone }\end{array}$ & Grainstone & Rudstone & Clay & Marl & Laminite & Dolomite \\
\hline \multirow[t]{2}{*}{ Min. } & 0.03 & 0.08 & 0.15 & 0.09 & \multirow[t]{2}{*}{0.11} & \multirow[t]{2}{*}{0.10} & & \multirow{3}{*}{1.32} & \multirow{3}{*}{$* * * *$} & \multirow{8}{*}{$\begin{array}{c}4.73 \\
45.96 \\
51 \\
0.90\end{array}$} & 0.38 \\
\hline & 0.09 & 0.02 & $* * * *$ & 0.06 & & & & & & & 9.64 \\
\hline Max. & 5.96 & 7.52 & 6.04 & 3.40 & 16.64 & 24.60 & 7.93 & & & & 171.28 \\
\hline Sum & 1113.73 & 272.95 & 136.34 & 49.4 & 103.69 & 339.32 & 122.48 & 5.12 & ***** & & 75 \\
\hline No. & 108 & 149 & 81 & 64 & 46 & 167 & 70 & 21 & ***** & & 2.28 \\
\hline Mean & 1.05 & 1.83 & 1.68 & 0.77 & 2.25 & 2.03 & 1.75 & 0.24 & $* * * *$ & & 13 \\
\hline \multirow[t]{2}{*}{$\%$} & 8 & 20 & 10 & 4 & 8 & 25 & & & & & \\
\hline & 9 & $<1$ & $* * * *$ & 3 & & & & & & & \\
\hline
\end{tabular}

The strata deeper than $798 \mathrm{mbsf}$ reflect the initial emergence and later flooding of the carbonate platform. The general flooding of the platform and evolution of a tidal-flat environment are revealed in the sediments of the interval from 798 to 1144 mbsf. From 798 to 853 mbsf, sediments are lagoonal (restricted shallow subtidal to intertidal) in origin (i.e., wackestone-packstone intervals with much clay). Algal mat disappears at top of this section, indicating a lowstand stage. The grainstones, abundant wackestone, and rudstone of the depth interval from 853 to 932 mbsf indicate a highstand stage, with maximum flooding at about 890 mbsf. From 932 to 1144 mbsf, mudstone, packstone, laminites, and clay reflect a restricted and shallow intertidal to supratidal environment. A grainstone at $1018 \mathrm{mbsf}$ having ripup clasts at its base (visible in the FMS image) is probably a storm deposit; similarly, other minor grainstones in this interval are probably washover.

The depth interval from 1144 to 1442 mbsf consists of shallow subtidal to intertidal facies showing a general shallowing upward of the platform. These facies are dolomitized. From 1144 to 1244 mbsf, wackestone-packstone sequences and abundant grainstone intervals indicate alternating progradation and aggradation. Throughout the interval from 1244 to 1442 mbsf, common grainstone with abundant wackestone-packstone indicate a highstand stage.

Two major intercalations of oolitic limestone are developed, one of latest Hauterivian age (Jenkyns et al., this volume), which rests on the basaltic edifice, and a second of Aptian age (Sager, Winterer, Firth, et al., 1993; Jenkyns et al., this volume) that is sandwiched between lagoonal-peritidal sediments (692-798 mbsf). The Hauterivian coarse, unsorted grainstones at the base of the section $(>1442$ mbsf) were deposited on an open-marine shelf or ramp.

In summary, FMS and geophysical logs of porosity and porosityrelated parameters (Fig. 10) confirm that the sedimentary record is composed of approximately 1 - to $10-\mathrm{m}$ sequences. Where resolution is good, smaller-scale sequences can be defined in the FMS logs, as well; often, however, the contrast is insufficient to identify them properly. These sequences are well developed at this site in the platform interior, where they probably reflect the cyclic deepening and shallowing of the depositional environment. From the lithostratigraphic interpretation of the logs and core information, we hypothesize that sediments of the upper $600 \mathrm{mbsf}$ of Hole $866 \mathrm{~A}$ originated in a somewhat restricted, tidal or subtidal environment, with oxygenated waters suitable for supporting a varied fauna. The predominant lithologies are upward-fining packstone-wackestone-mudstone sequences that have some significant grainstone intervals. Strasser (this volume) suggests a potential hiatus or condensed section at 480 mbsf, based on occurrence of calcrete horizons; the FMS logs place these horizons at a slightly different depth, about 465 mbsf. From about 600 mbsf downward, lithologies indicate a restricted lagoonal environment, punctuated by intervals of slightly more open lagoonal environment of up to $100 \mathrm{~m}$ thick (750-850, 880-930, 1180-1230, and 1260-1340 mbsf). Normal open-marine conditions prevail below $1340 \mathrm{mbsf}$.

\section{COMPARISON OF ALBIAN SECTIONS AT SITES 865 AND 866}

Beds for sections of Albian age were grouped into upward-fining (e.g., packstone-wackestone-mudstone) or upward-coarsening sequences. A plot of the thicknesses of upward-fining sequences vs. their centered depths (halfway between base and top) for both holes is shown in Figure 11. The plots should be regarded as qualitative and are not meant to provide the basis for a quantitative comparison; some beds contain numerous interbeds of clay or laminite, indicating numerous minor cycles, for example, and no attempt was made to measure beds thinner than $1 \mathrm{~cm}$. Cycle thicknesses for Hole 865A are generally greater than those for Hole $866 \mathrm{~A}$; this is in agreement with the greater sedimentation rates suggested for Hole 865A (Sager, Winterer, Firth, et al., 1993). The thickest sequence measured for Hole $865 \mathrm{~A}$, located from 364.1 to 409.2 mbsf (packstone grading up to wackestone with extreme moldic porosity), is close in depth to that of Hole 866A, 411.9 to 442.0 mbsf (packstone grading up to mudstone with extreme moldic porosity). Could these two sequences be equal in time, especially because sequences above this level are generally longer for both holes, and sequences below this level are generally shorter for both holes? Evidence in support of this contention is found in logging "events" at corresponding depths at both sites. These events consist of high-amplitude excursions from prevailing values, usually in the resistivity $\log$, occasionally seen in the natural gamma-ray log. At Site 865 (Fig. 9), such events occur at 240, 272,305 , and $320 \mathrm{mbsf}$, whereas at Site 866 (Fig. 10), similar events occur at $280,325,350$, and 363 mbsf. Thus, deposition of the lagoonal sediments that make up the Albian section at Sites 866 and 865 probably overlapped somewhat. If this is the case, then, from Figure 11 , it appears that much of the late Albian section above the 400 -mbsf level is missing at Hole $865 \mathrm{~A}$, perhaps removed as the guyot emerged above sea level. At Hole $866 \mathrm{~A}$, a hiatus probably exists at the base of the Albian section (Strasser, this volume).

\section{CONCLUSIONS}

Deep holes drilled into the Cretaceous lagoonal facies of Allison (Site 865) and Resolution (Site 866) guyots yielded samples of thick sections of shallow-water limestones that record the histories of the 


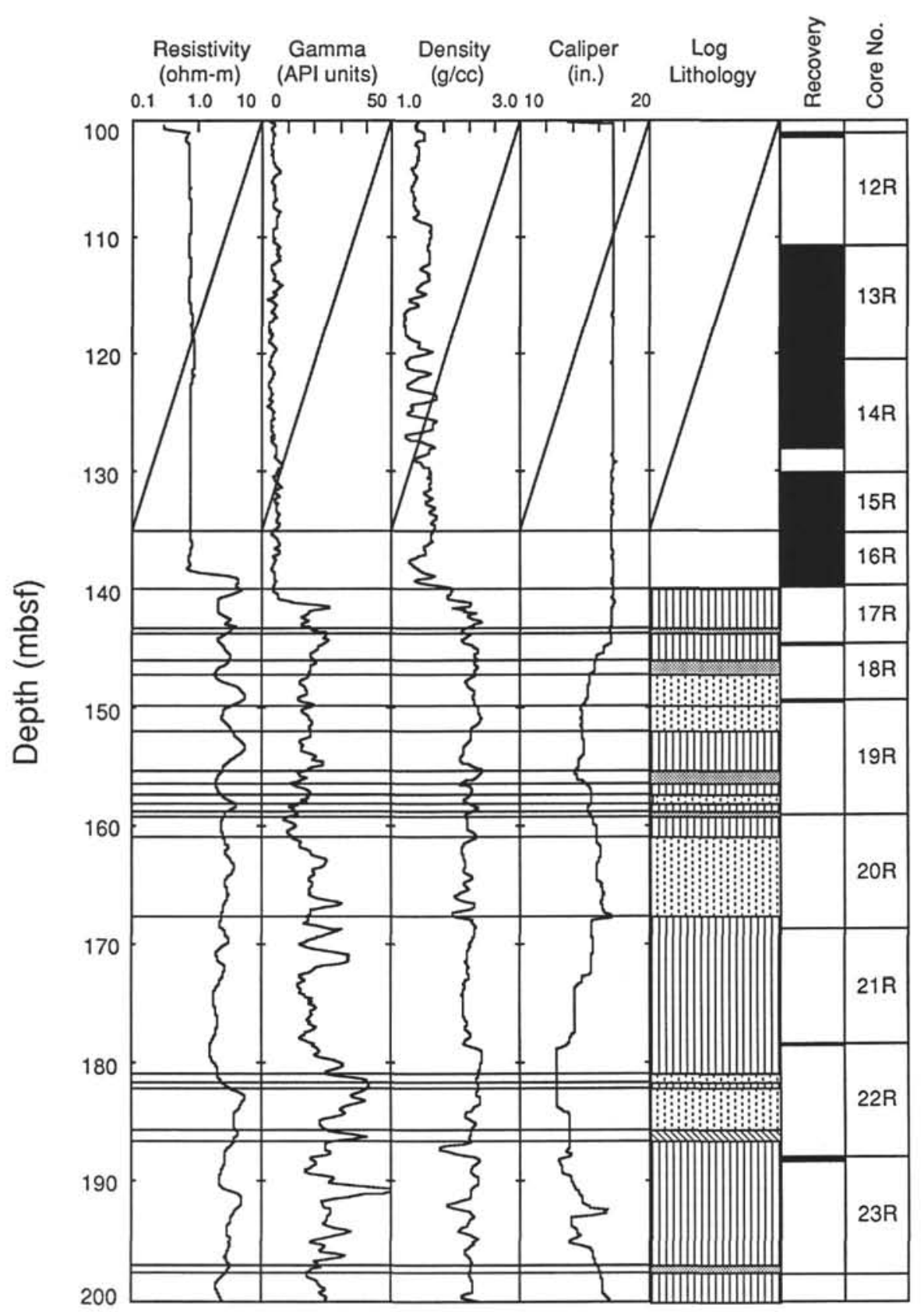

Figure 9. Profiles of (from left) medium electrical resistivity (ILM), natural gamma-ray intensity (NGT), formation density (RHOB), and caliper (HLDT), determined from downhole logs at Hole 865A. Cored intervals and recovery (in black) are shown in the right columns. The exact position of the cored material within the cored intervals is not constrained; recovered core material was pushed to the top of each cored interval for consistency. Also shown are lithologic interpretations based on integration of the FMS and geophysical logs with core descriptions; the pattern legend is shown in Figure 8.

guyots from initial submergence of the volcanic pedestal through the final drowning of the carbonate platform. However, core recovery was poor, and we suspect that not all lithologies were sampled and, further, that studies of widely spaced core samples would not provide a sufficiently detailed record of the vertical trajectories of the guyots for correlating directly between them. As downhole logs provide a more continuous record of the physical properties of the borehole, the integration of logging data with core descriptions presents a lithologic record that is both detailed and accurate.

Facies that evoked similar electrical responses in the conventional and FMS logs were calibrated using laboratory descriptions and photographs of recovered core materials to produce "type" examples for the various carbonate facies. The type examples then were used to convert the remaining logging data into lithologic columns. We saw immediately that core recovery at both sites was highly preferential (e.g., within the alternating packstone-wackestone intervals, only small pebbles of well-cemented wackestone were recovered). This study indicated that packstone was the dominant lithology $(51 \%)$ at Hole $865 \mathrm{~A}$, and that grainstone $(25 \%)$ and packstone $(20 \%)$ were the dominant lithologies at Hole 866A (excluding dolomite). Recovery of packstone and grainstone was poor.

The sedimentary record from Allison Guyot, as determined from core-log integration, confirmed the existence of numerous smallscale shallow-water carbonate sequences of (late) Albian age. Facies from the lower part of the hole imply a clay- and organic-rich, marshy environment with a nearby landmass, gradually opening uphole into 


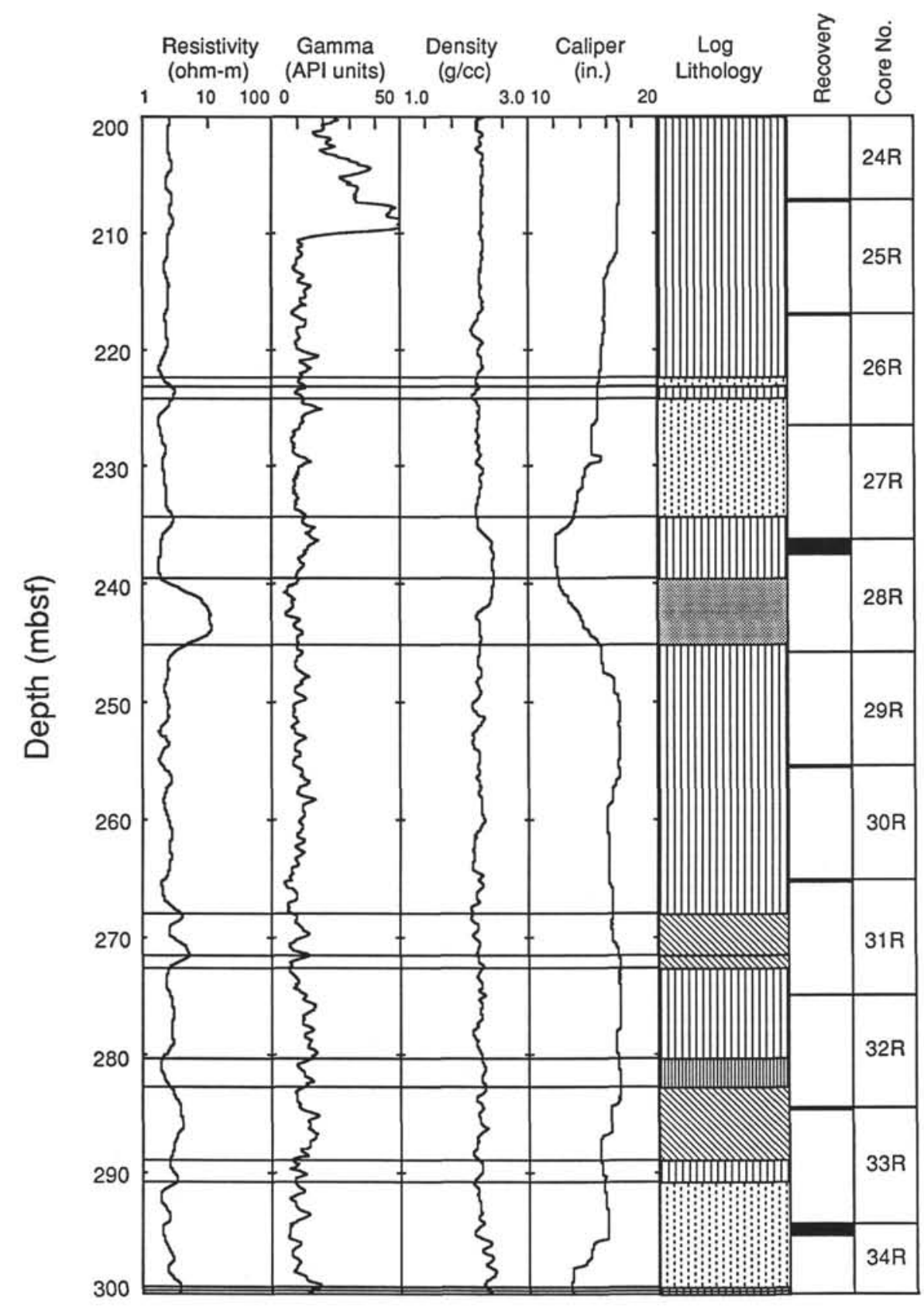

Figure 9 (continued).

a somewhat restricted lagoonal setting, similar to the Albian (200550 mbsf) section at Hole 866A.

Quality of logs was better at Resolution Guyot because of better hole conditions. The sedimentary record at Hole 866A was composed of sequences 1 to $10 \mathrm{~m}$ long. Where resolution was particularly good, smaller-scale sequences could be defined in the FMS logs as well, although the contrast was often insufficient to identify them properly. Sediments in the upper 600 mbsf of Hole $866 \mathrm{~A}$ originated in a somewhat restricted tidal or subtidal environment. Lithologies at depths greater than $600 \mathrm{mbsf}$ imply a restricted lagoonal environment with occasional periods of more open lagoonal conditions. Lithologies below 1340 mbsf suggest normal open-marine conditions.

Lithologies were grouped into upward-fining sequences at both localities.

Sequence thicknesses for Hole $865 \mathrm{~A}$ generally are greater than those for Hole 866A; this is in agreement with the greater sedimenta- tion rates suggested for Hole 865A (Sager, Winterer, Firth, et al., 1993). The thickest sequence measured for Hole $865 \mathrm{~A}$, located at 364.1 to 409.2 mbsf (packstone grading upward to wackestone with extreme moldic porosity), corresponds in depth to that of Hole 866A at 411.9 to 442.0 mbsf (packstone grading upward to mudstone with extreme moldic porosity). Support for this contention is found in the existence of several smaller-scale, but significant, logging "events" found at similar depths in both holes. At both sites, this thick sequence defines a change in either depositional style or subsidence rate as sequences above this level are generally thicker, and sequences below this level are generally thinner, for both holes. This study supports Strasser's conclusion (this volume) that a hiatus probably exists near the Albian/Aptian boundary ( $480 \mathrm{mbsf})$. Correlations of bed thicknesses and logging signatures between the two holes indicate that much of the Albian section may have been removed from Allison Guyot as it emerged above sea level. 


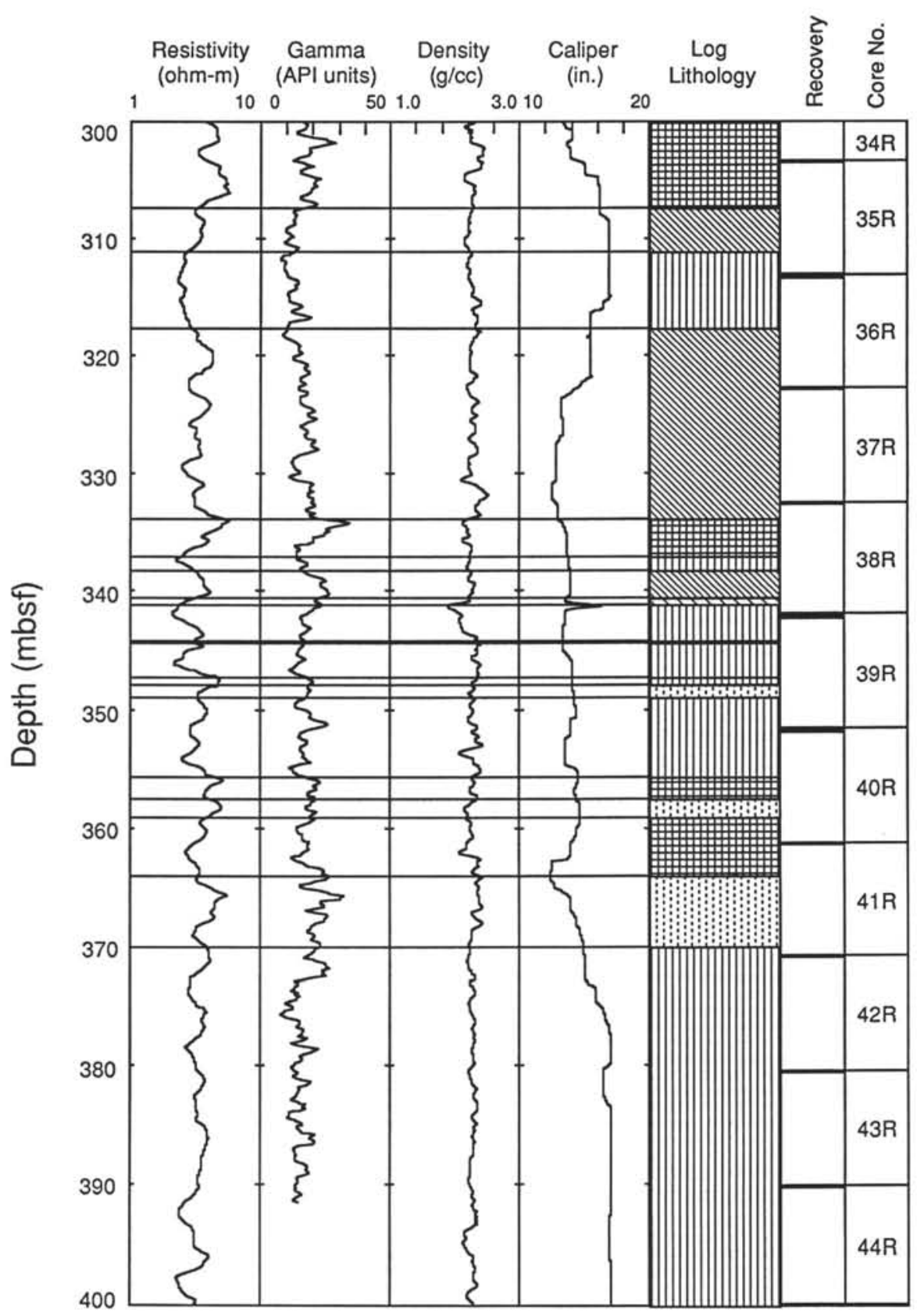

Figure 9 (continued).

\section{ACKNOWLEDGMENTS}

Preliminary advice to P. Cooper on presentation and interpretation of data by E.L. Winterer, W. Sager, and J. Ogg is greatly appreciated. We are grateful to Hervé Cambray, Will Sager, and an anonymous reviewer for their helpful suggestions. This is SOEST Contribution No. 3550 .

\section{REFERENCES $*$}

Adams, J., Bourke, L., and Buck, S., 1990. Integrating Formation MicroScanner images and cores. Oilfield Rev., 2:52-65.

\footnotetext{
- Abbreviations for names of organizations and publications in ODP reference lists follow the style given in Chemical Abstracts Service Source Index (published by American Chemical Society).
}

Archie, G.E., 1942. The electrical resistivity log as an aid in determining some reservoir characteristics. Trans. Am. Inst. Min., Metall. Pet. Eng., 146:54-62. , 1952. Classification of carbonate reservoir rocks and petrophysical considerations. AAPG Bull., 36:278-298.

Bourke, L., Delfiner, P., Trouiller, J.-C., Fett, T., Grace, M., Lüthi, S., Serra, O., and Standen, E., 1989. Using Formation MicroScanner images. Tech. Rev., 37:16-40.

Dunham, R.J., 1962. Classification of carbonate rocks according to depositional texture. In Ham, W.E. (Ed.), Classification of Carbonate Rocks. AAPG Mem., 1:108-121.

Ekstrom, M.P., Dahan, C., Chen, M.-Y., Lloyd, P., and Rossi, D.J., 1987. Formation imaging with microelectrical scanning arrays. Log Analyst, 28:294-306.

Harker, S.D., McGann, G.J., Bourke, L.T., and Adams, J.T., 1990. Methodology of Formation Micro Scanner image interpretation in Claymore and Scapa Fields (North Sea). In Hurst, A., Lovell, M.A., and Morton, A.C. (Eds.), Geological Applications of Wireline Logs. Geol. Soc. Spec. Publ. London, 48:11-25. 


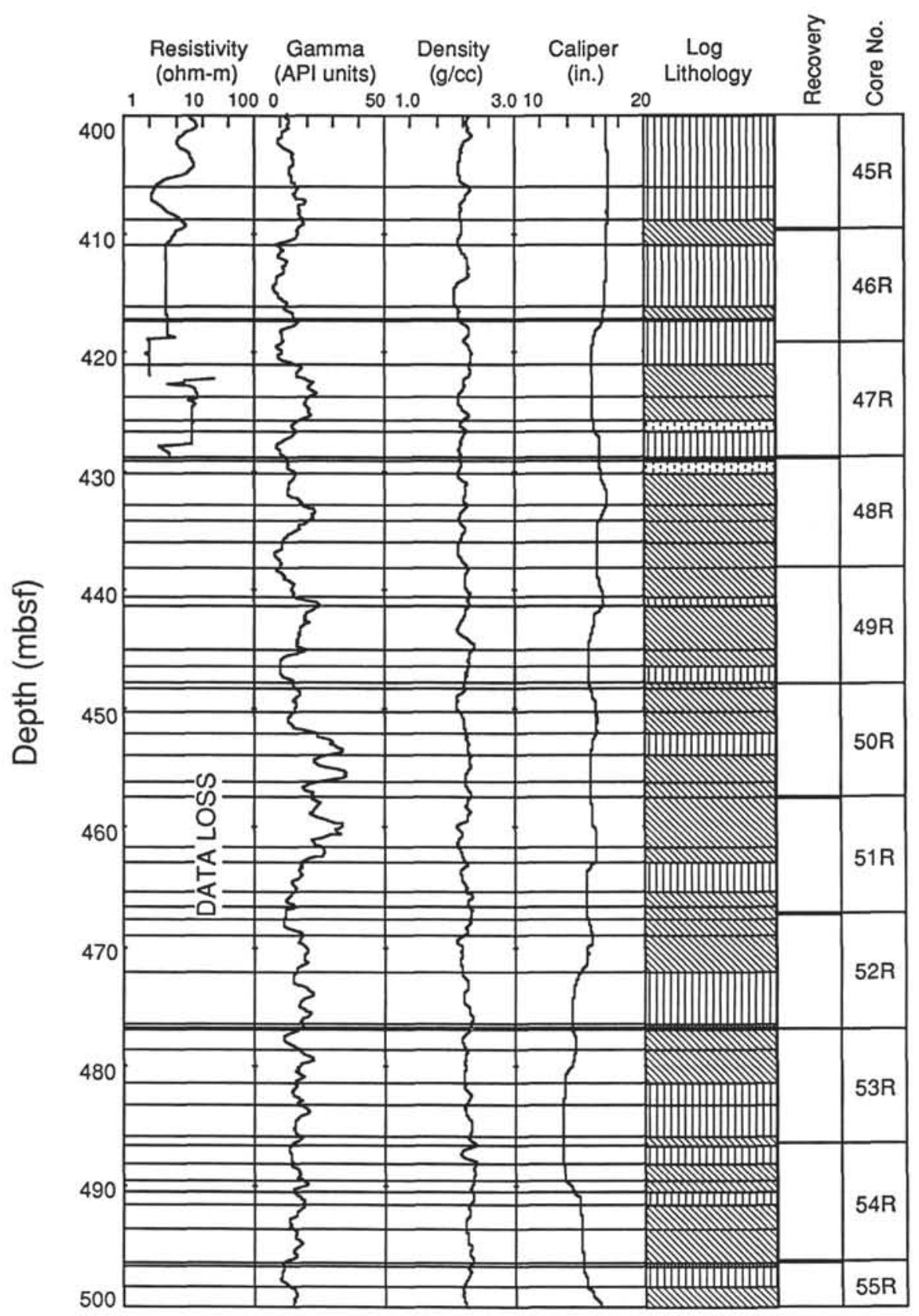

Figure 9 (continued).

James, N.P., 1989. Structure and stratigraphy of modern and ancient reefs. In Scholle, P.A., James, N.P., and Read, J.F. (Eds.), Carbonate Sedimentology and Petrology. Short Course, Am. Geophys. Union, 4:1-40.

Luthi, S.M., 1990. Sedimentary structures of clastic rocks identified from electrical borehole images. In Hurst, A., Lovell, M.A., and Morton, A.C. (Eds.), Geological Applications of Wireline Logs. Geol. Soc. Spec. Publ. London, 48:3-10.

Matthews, J.L., Heezen, B.C., Catalano, R., Coogan, A., Tharp, M., Natland, J., and Rawson, M., 1974. Cretaceous drowning of reefs on Mid-Pacific and Japanese guyots. Science, 184:462-464.

McNutt, M.K., Winterer, E.L., Sager, W.W., Natland, J.H., and Ito, G., 1990. The Darwin Rise: a Cretaceous superswell? Geophys. Res. Lett., 17:11011104.

Menard, H.W., 1964. Marine Geology of the Pacific: New York (McGrawHill).
Sager, W.W., Winterer, E.L., Firth, J.V., et al., 1993. Proc. ODP, Init. Repts., 143: College Station, TX (Ocean Drilling Program).

Serra, O., 1989. Formation MicroScanner Image Interpretation: Houston (Schlumberger Educ. Services), SMP-7028.

Shipboard Scientific Party, 1993. Introduction and scientific objectives. In Sager, W.W., Winterer, E.L., Firth, J.V., et al., Proc. ODP, Init. Repts., 143: College Station, TX (Ocean Drilling Program), 7-12.

Winterer, E.L., and Metzler, C.V., 1984. Origin and subsidence of guyots in Mid-Pacific Mountains. J. Geophys. Res., 89:9969-9979.

Date of initial receipt: 1 December 1993

Date of acceptance: 5 July 1994

Ms 143SR-237 


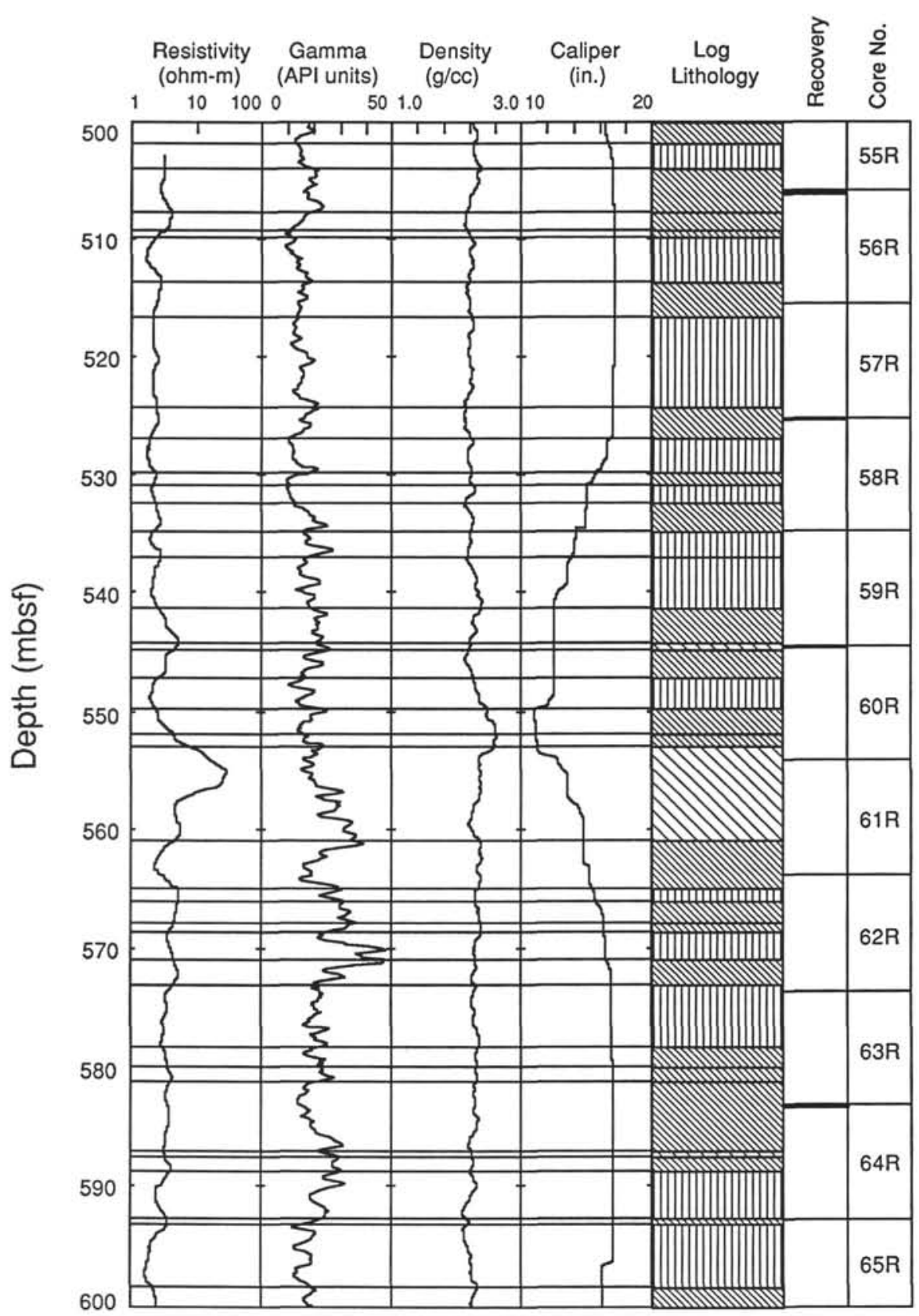

Figure 9 (continued). 


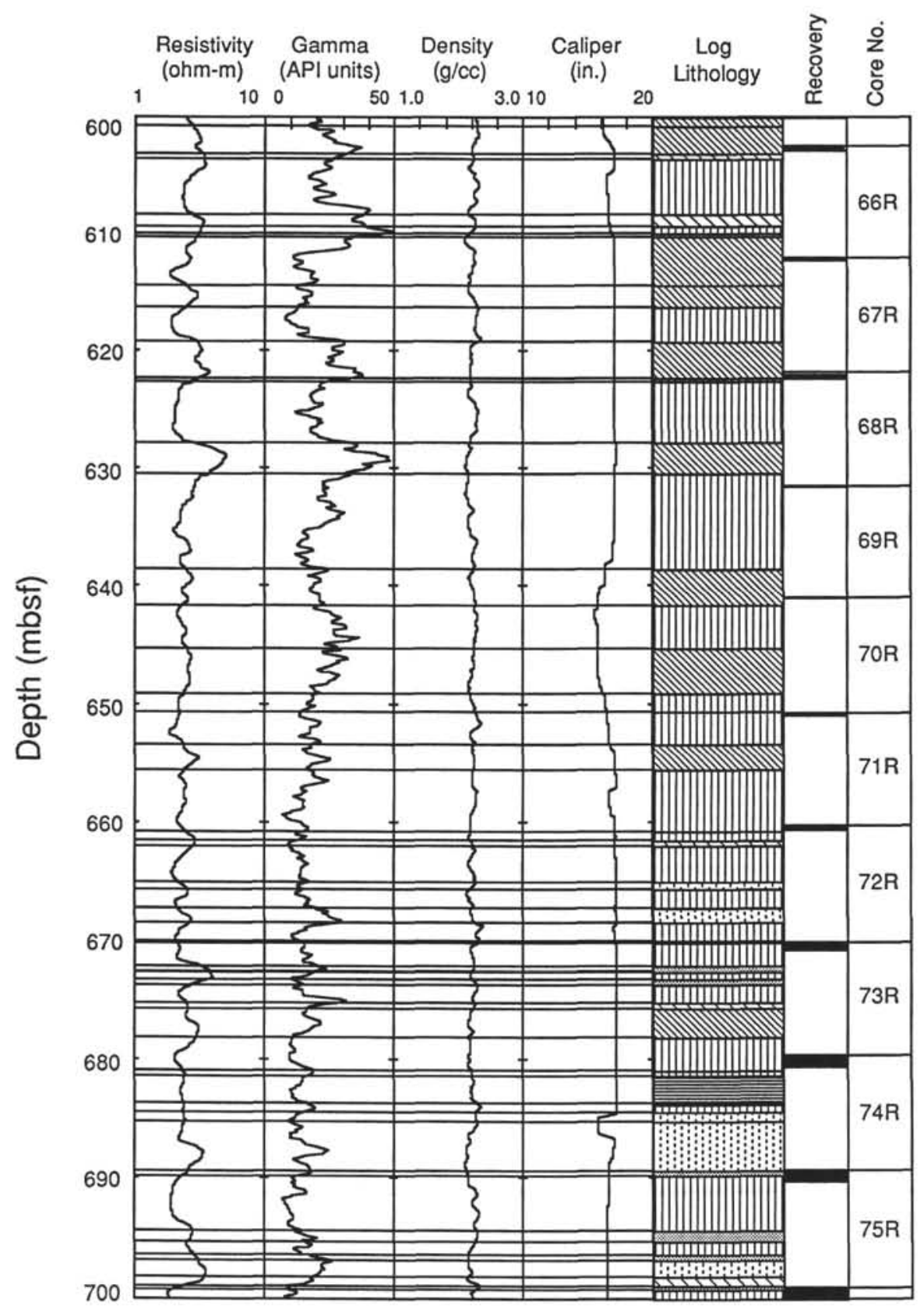

Figure 9 (continued). 


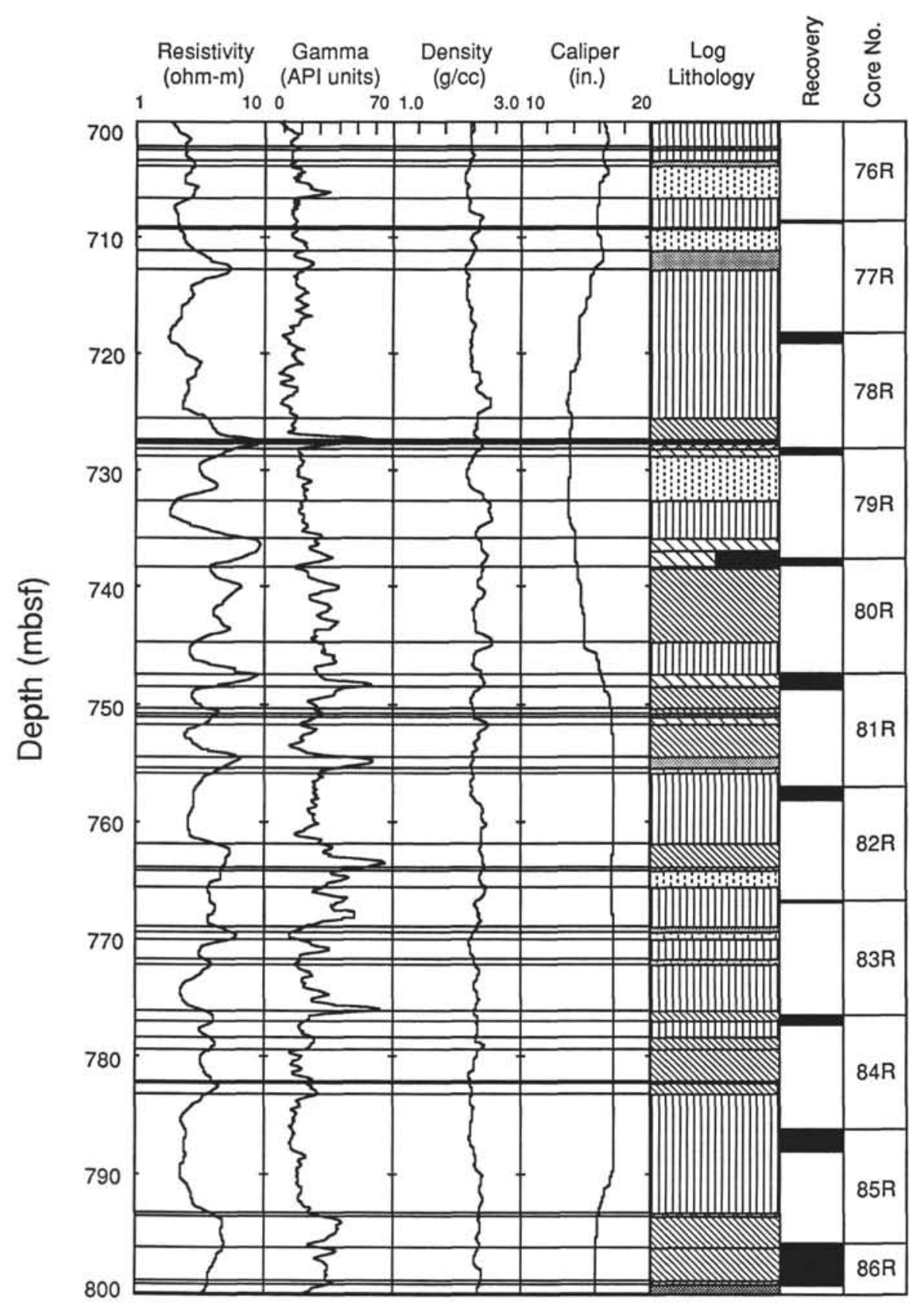

Figure 9 (continued). 


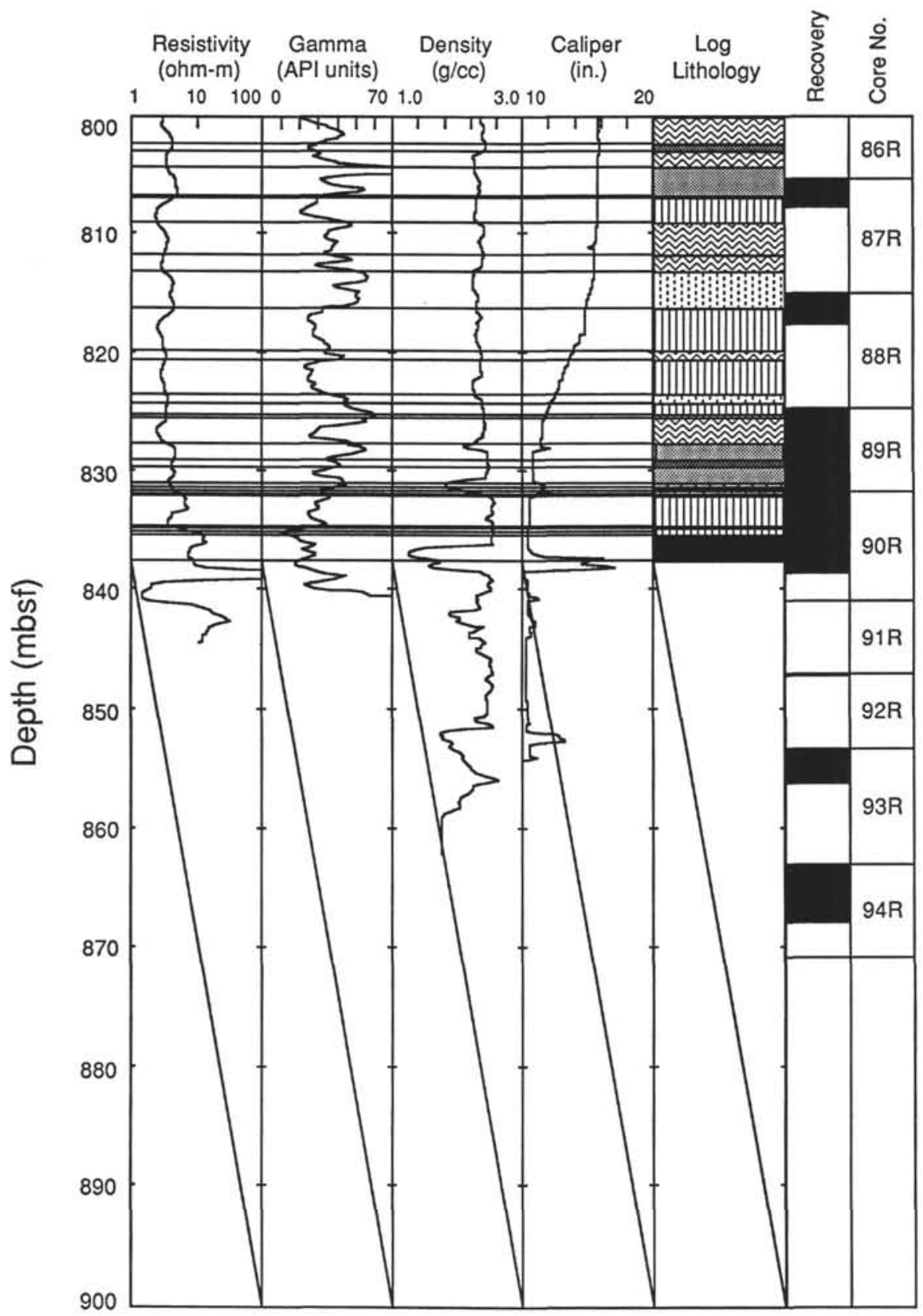

Figure 9 (continued). 


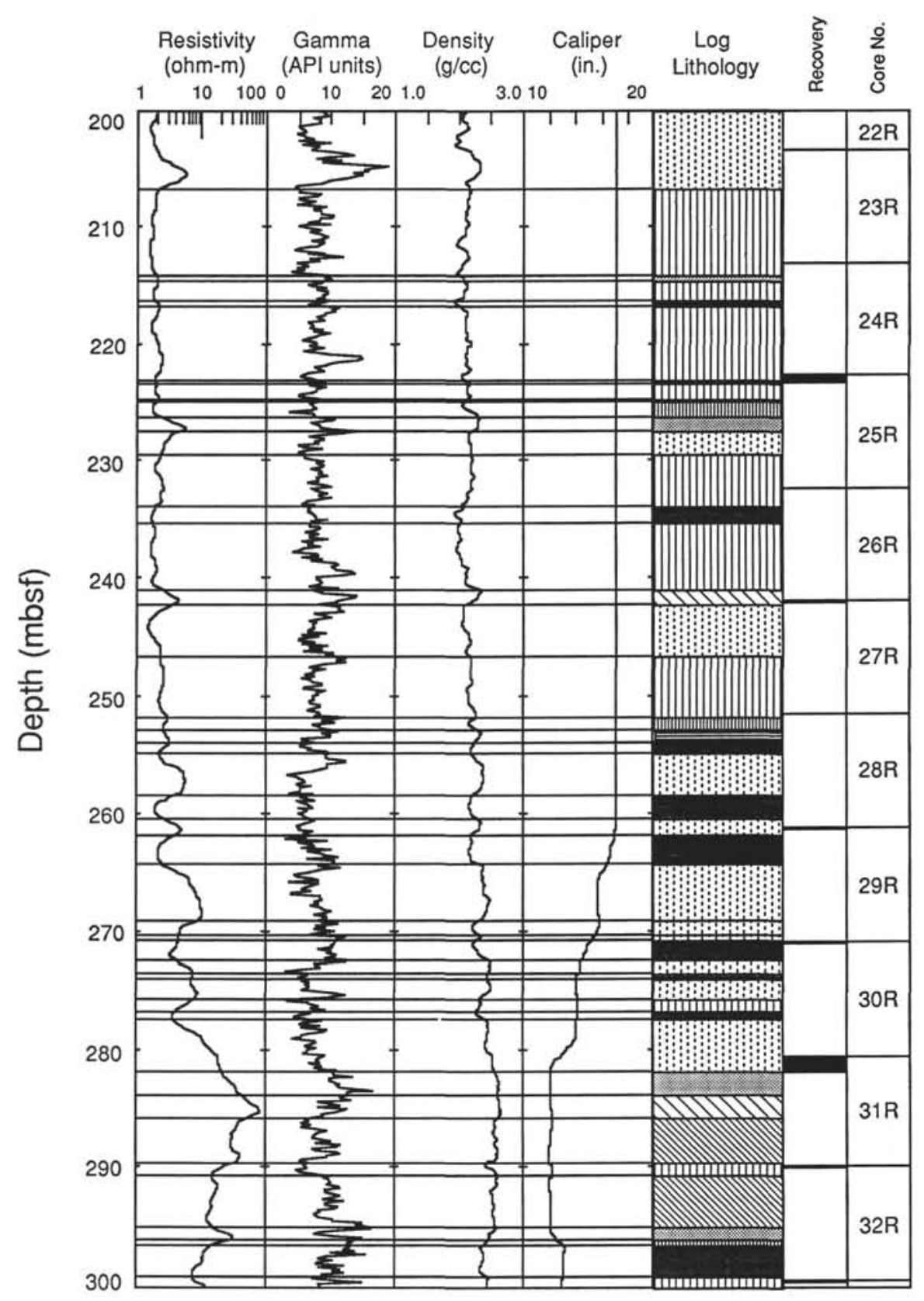

Figure 10. Profiles of (from left) medium electrical resistivity (ILM), natural gamma-ray intensity (NGT), formation density (RHOB), and caliper (HLDT), determined from downhole logs at Hole 866A. Cored intervals and recovery (in black) are shown in the right columns. The exact position of the cored material within the cored intervals is not constrained; recovered core material was pushed to the top of each cored interval for consistency. Also shown are lithologic interpretations based on integration of the FMS and geophysical logs with core descriptions; the pattern legend is shown in Figure 8. 


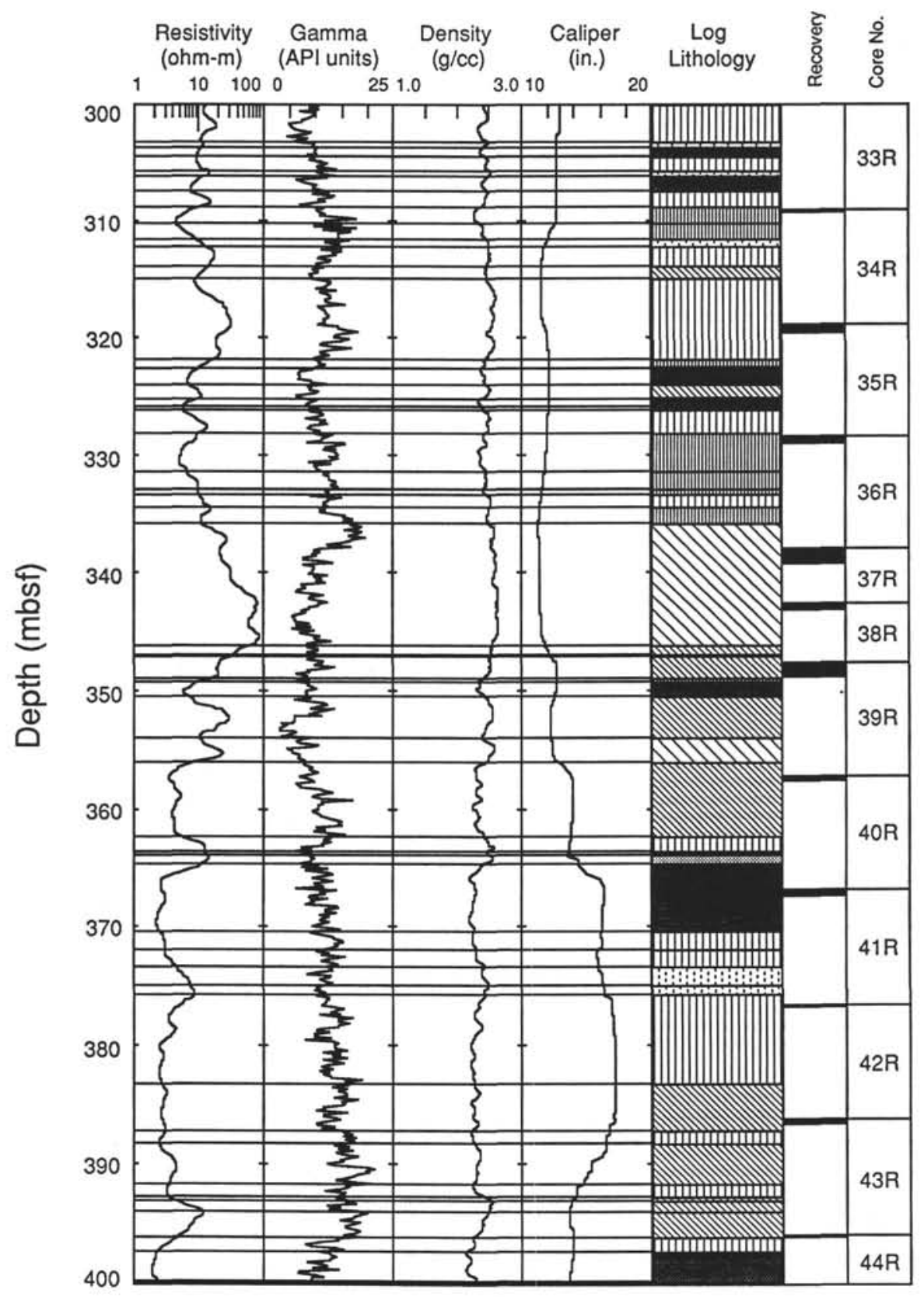

Figure 10 (continued). 


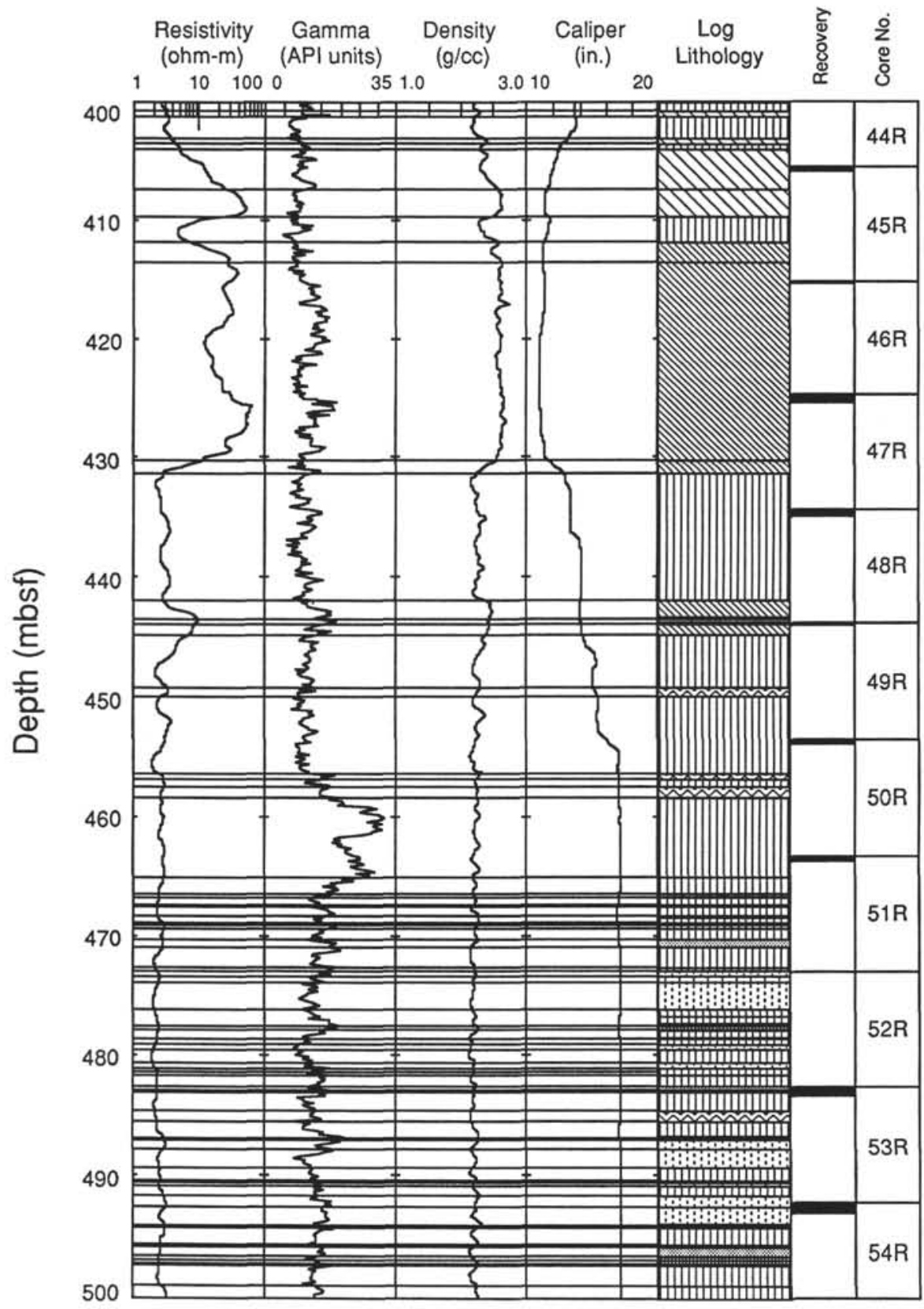

Figure 10 (continued). 


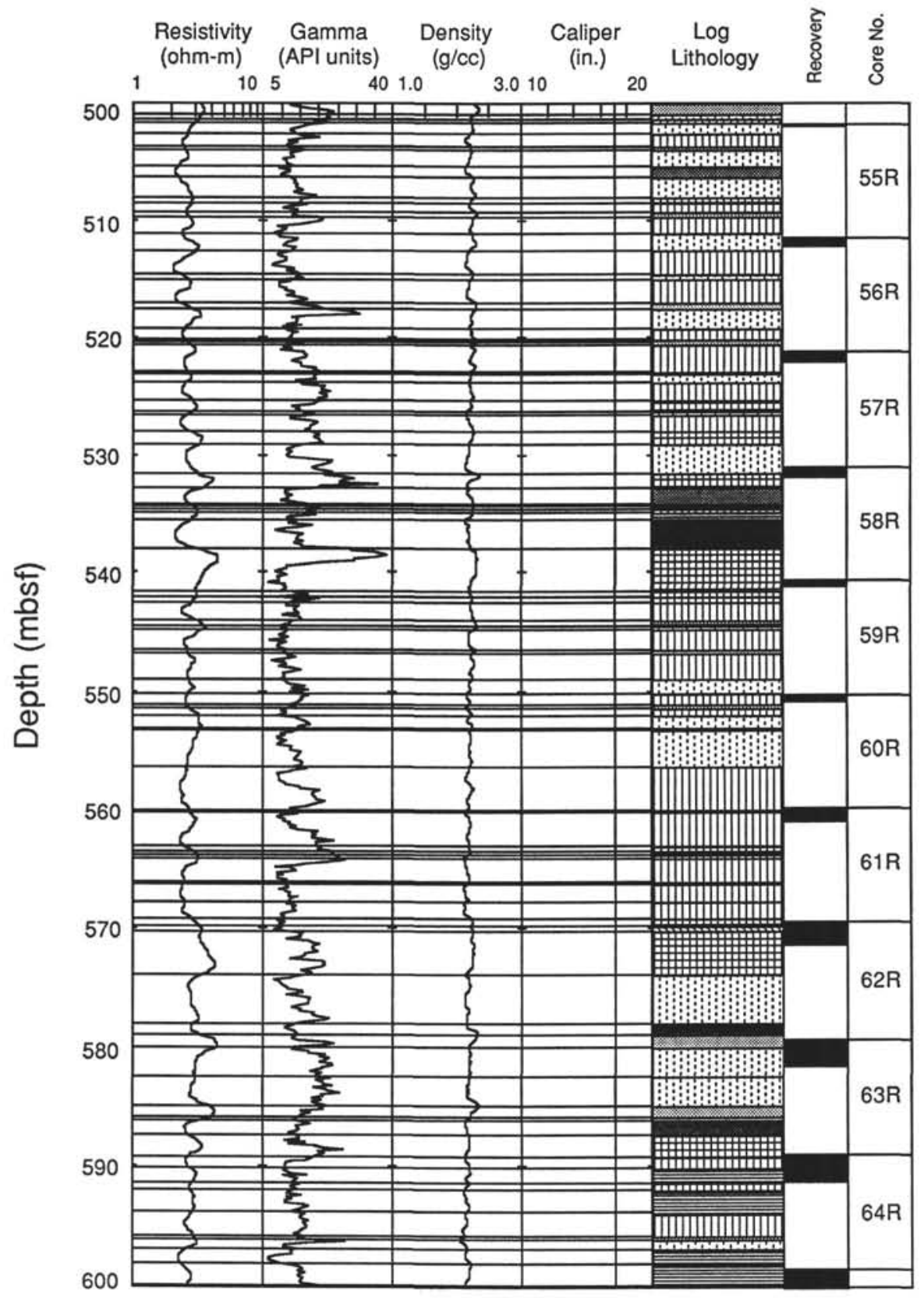

Figure 10 (continued). 


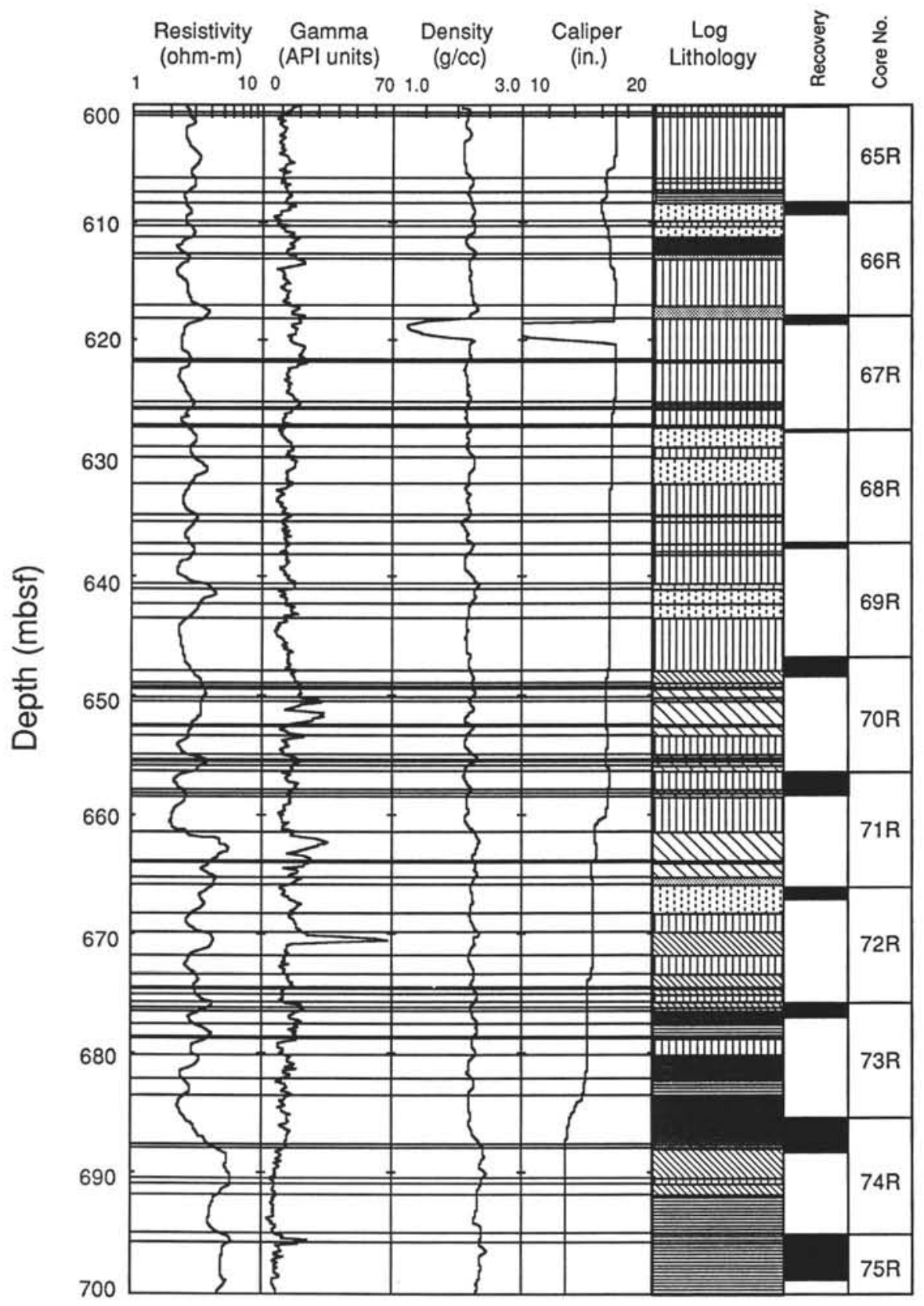

Figure 10 (continued). 


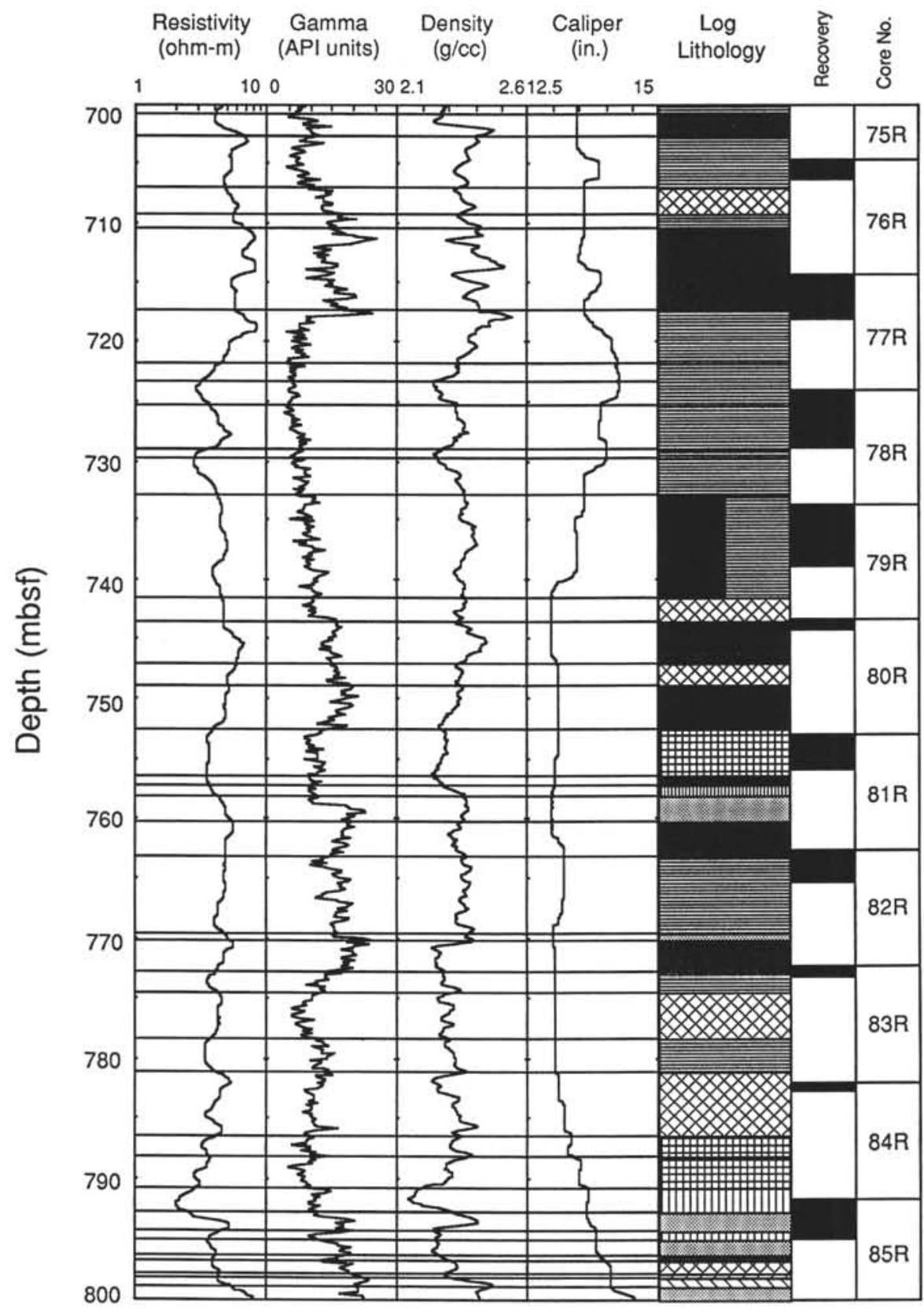

Figure 10 (continued). 


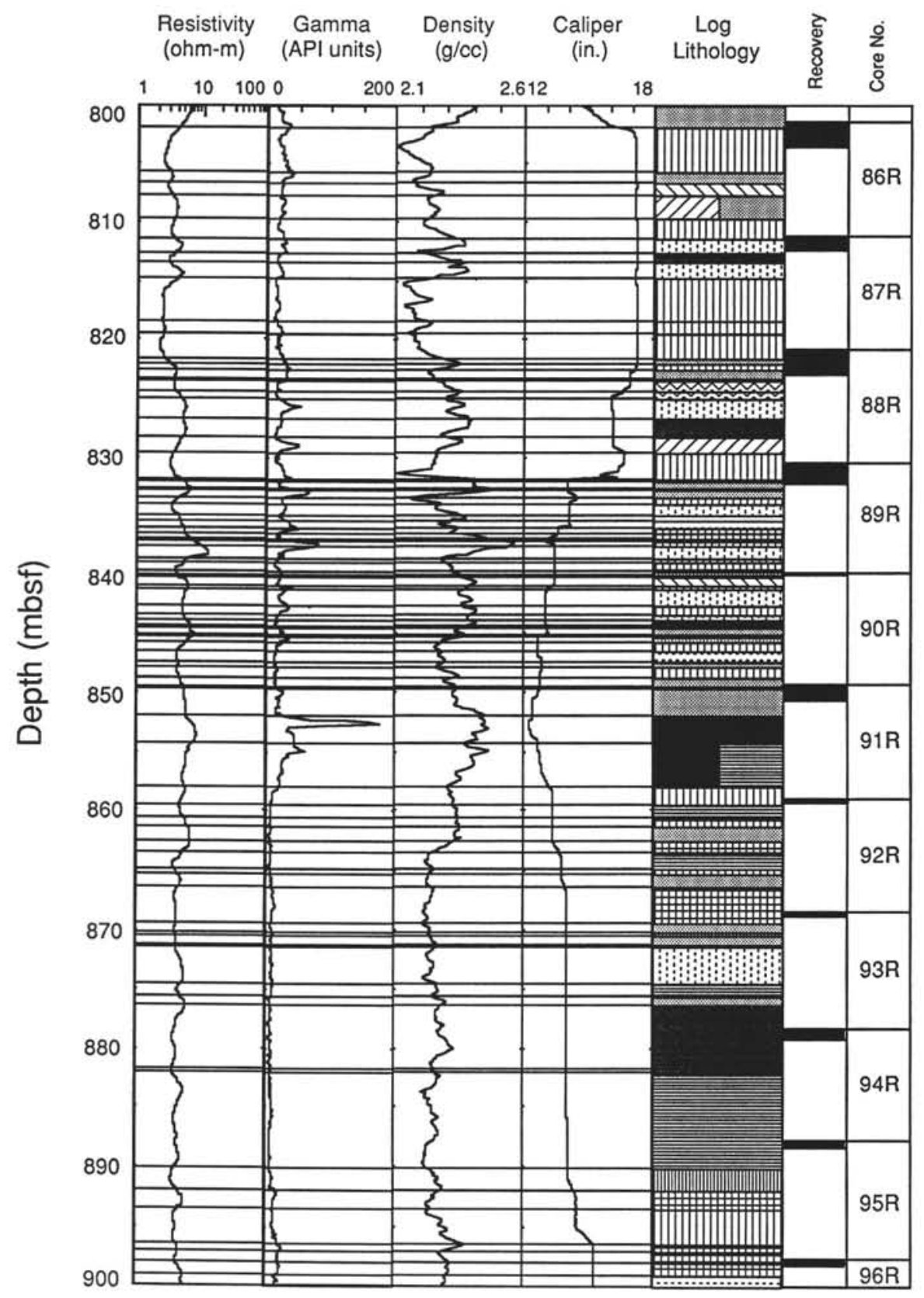

Figure 10 (continued). 


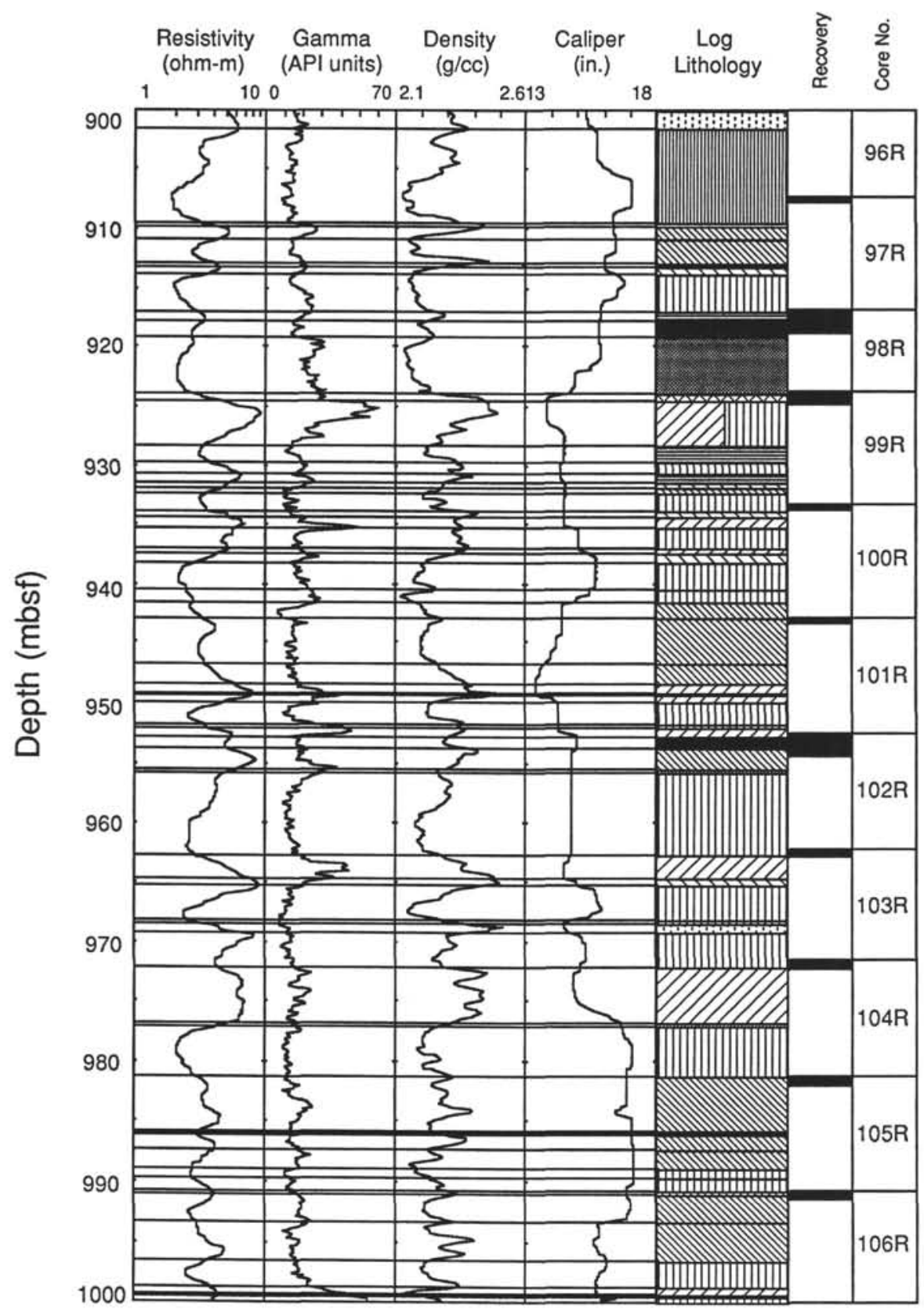

Figure 10 (continued). 


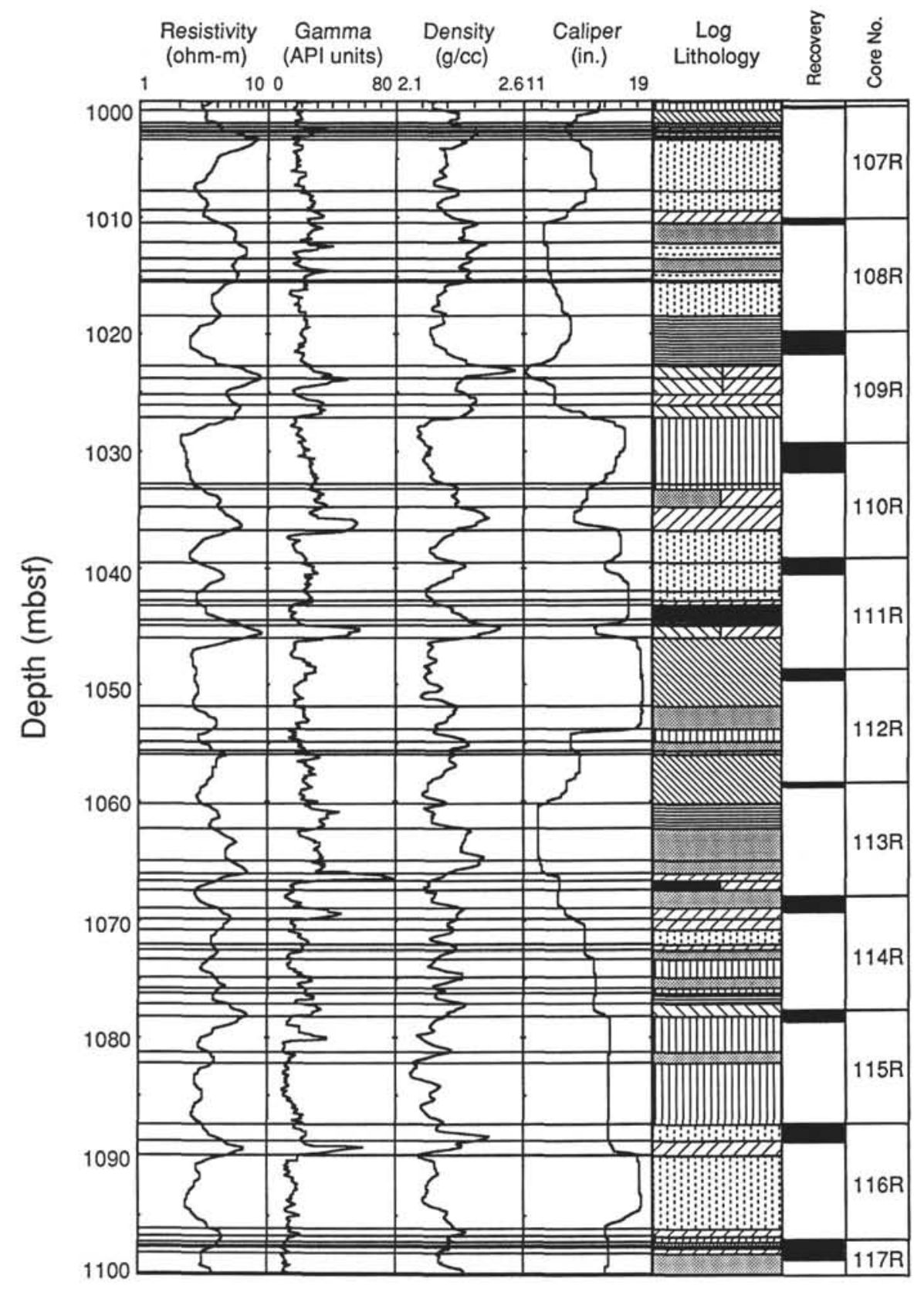

Figure 10 (continued). 


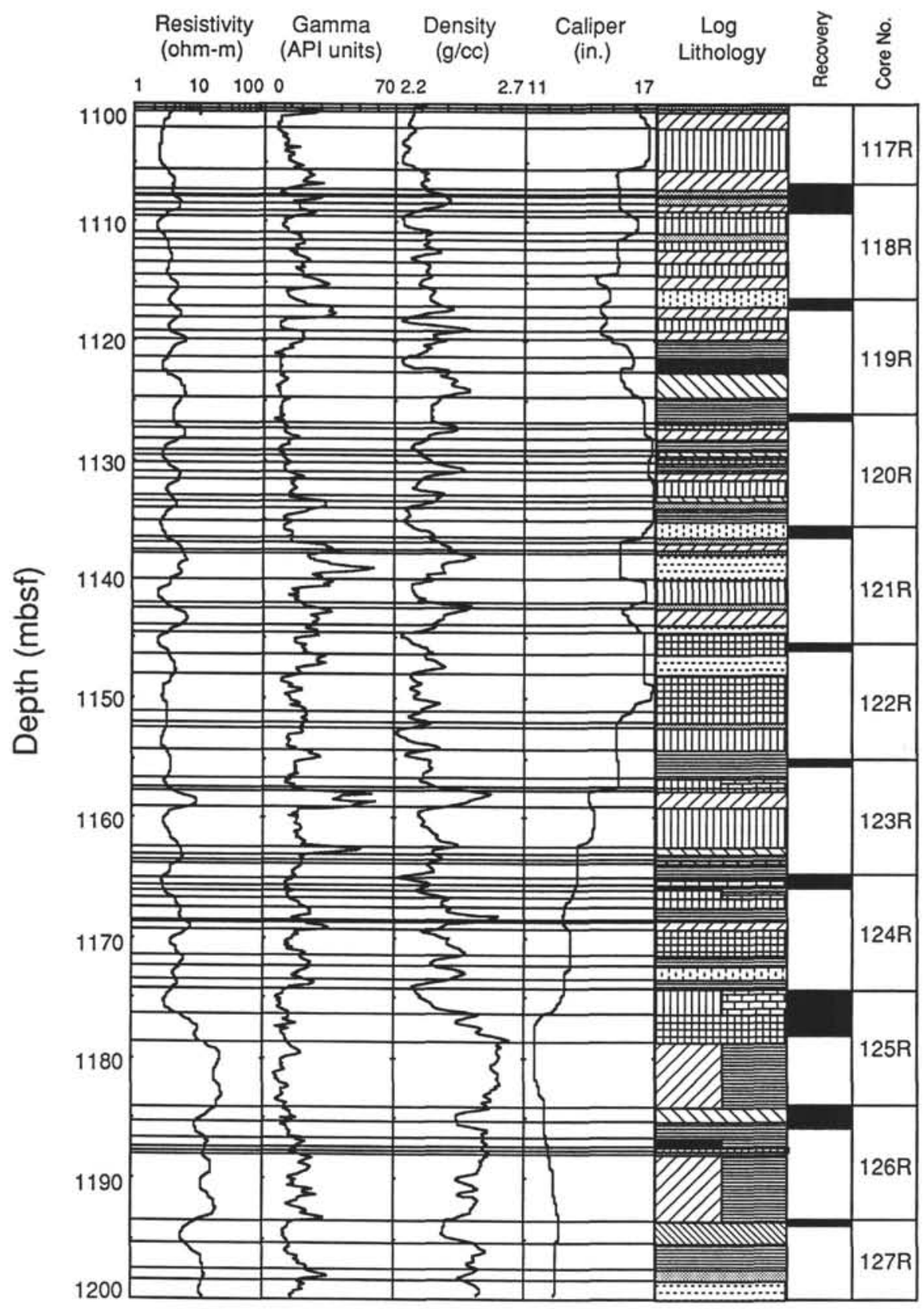

Figure 10 (continued). 


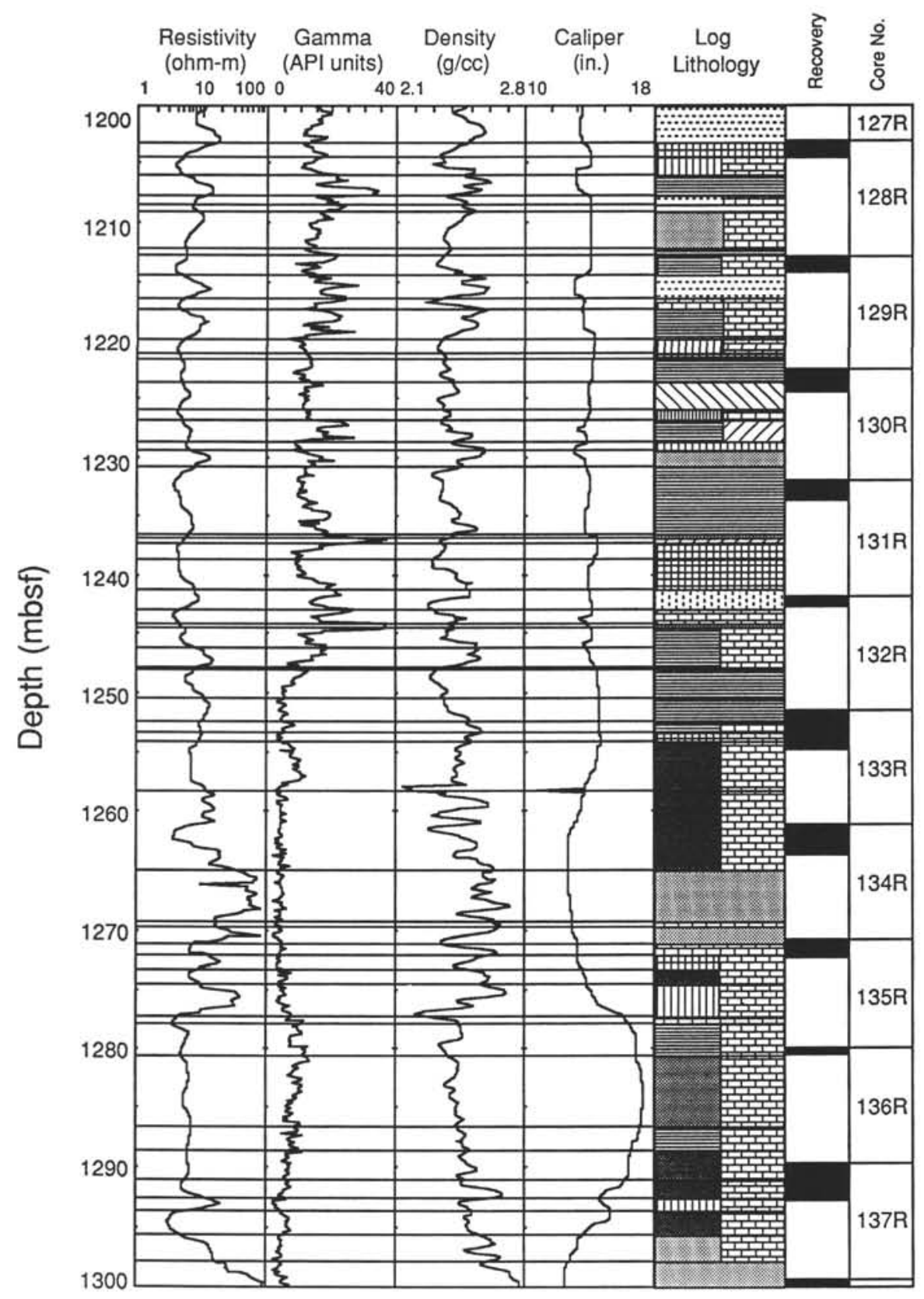

Figure 10 (continued). 


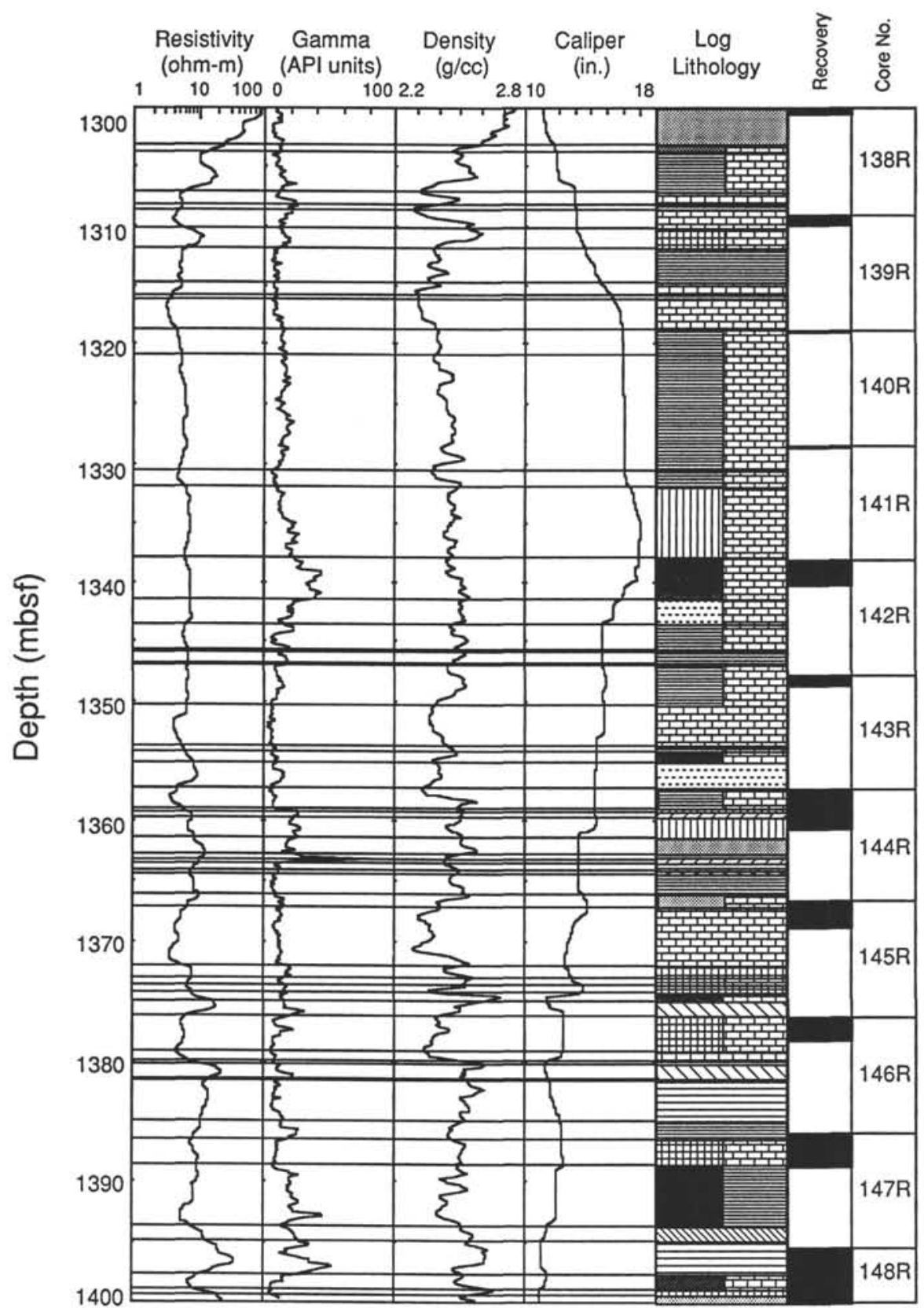

Figure 10 (continued). 


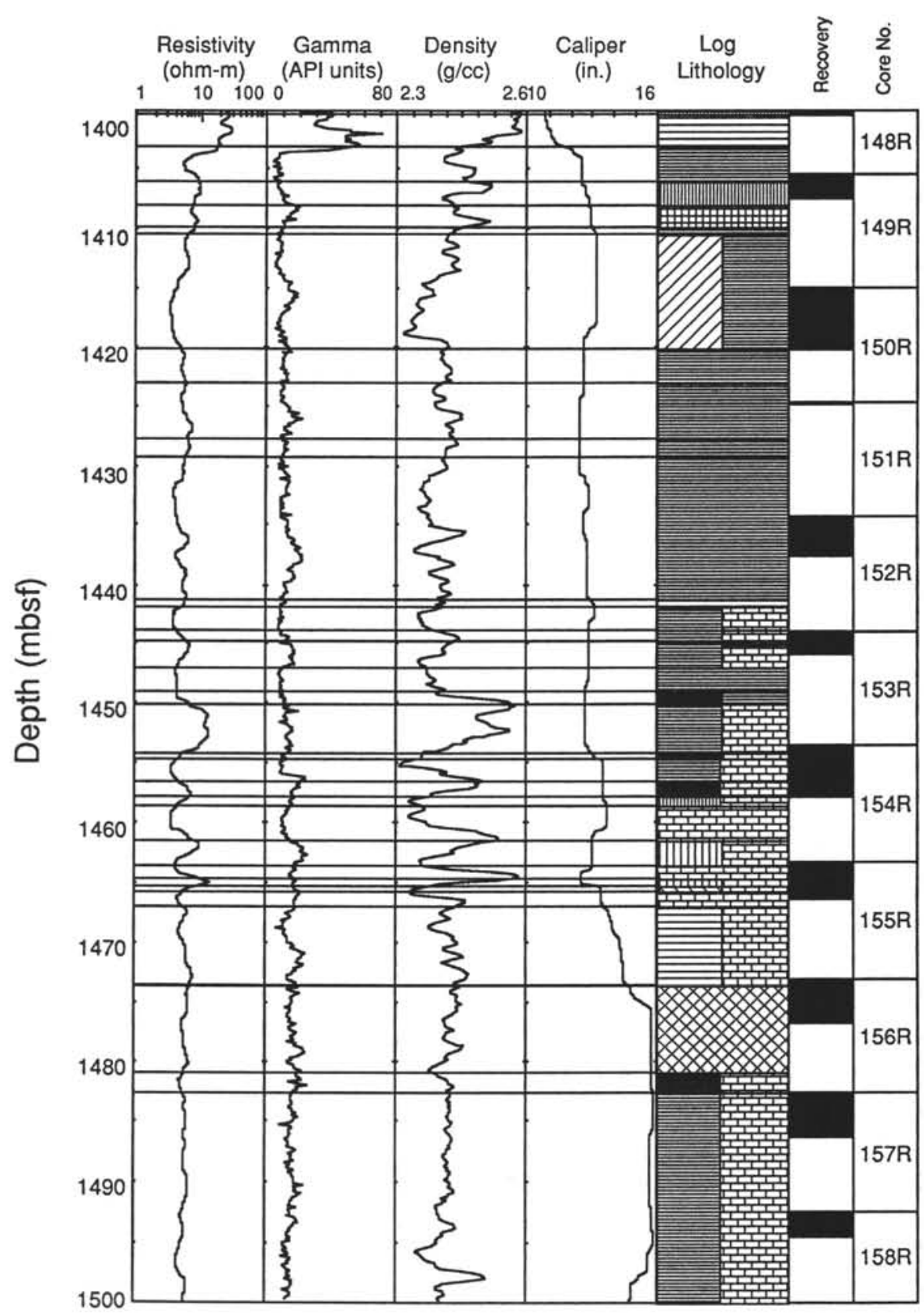

Figure 10 (continued). 


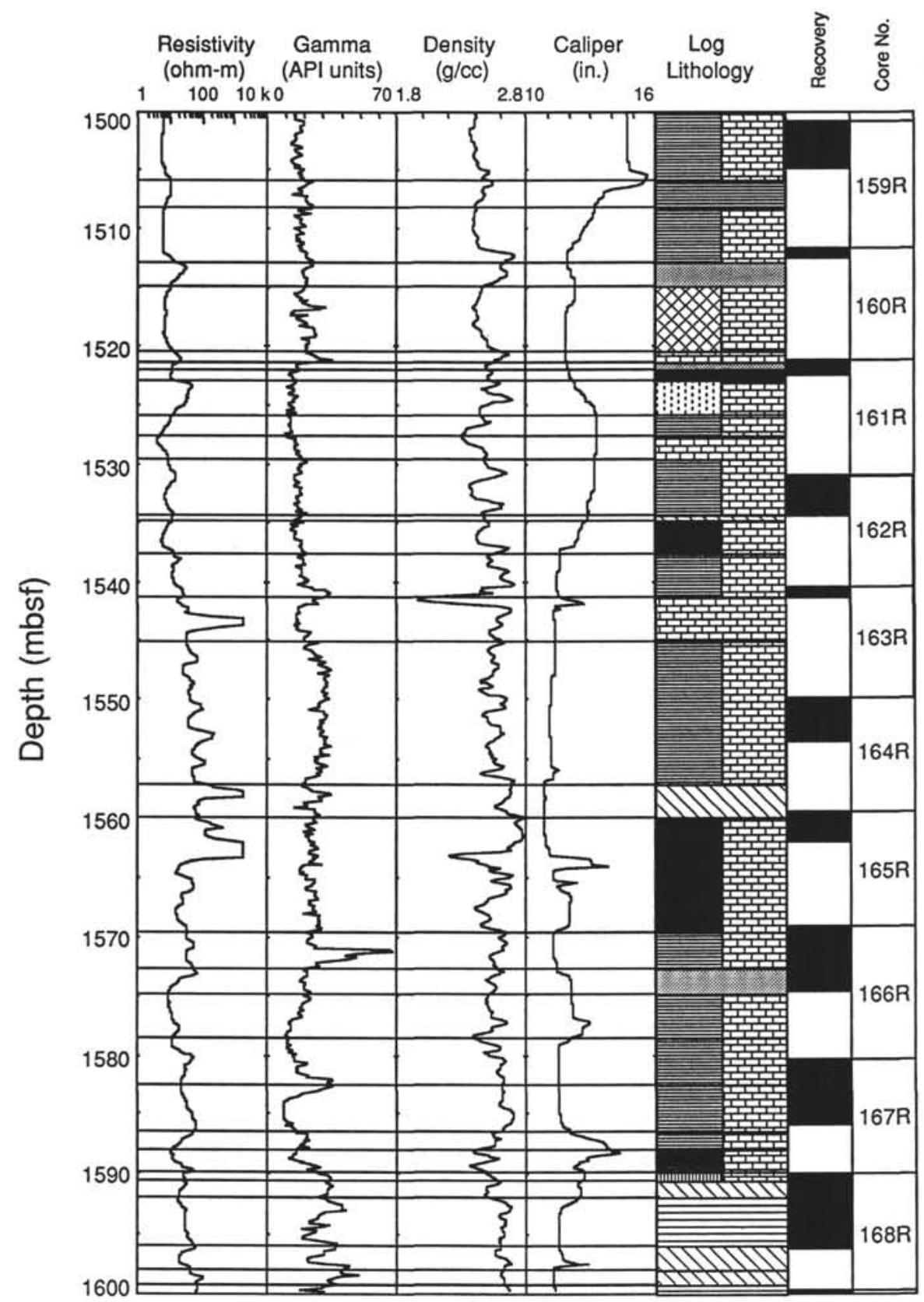

Figure 10 (continued). 


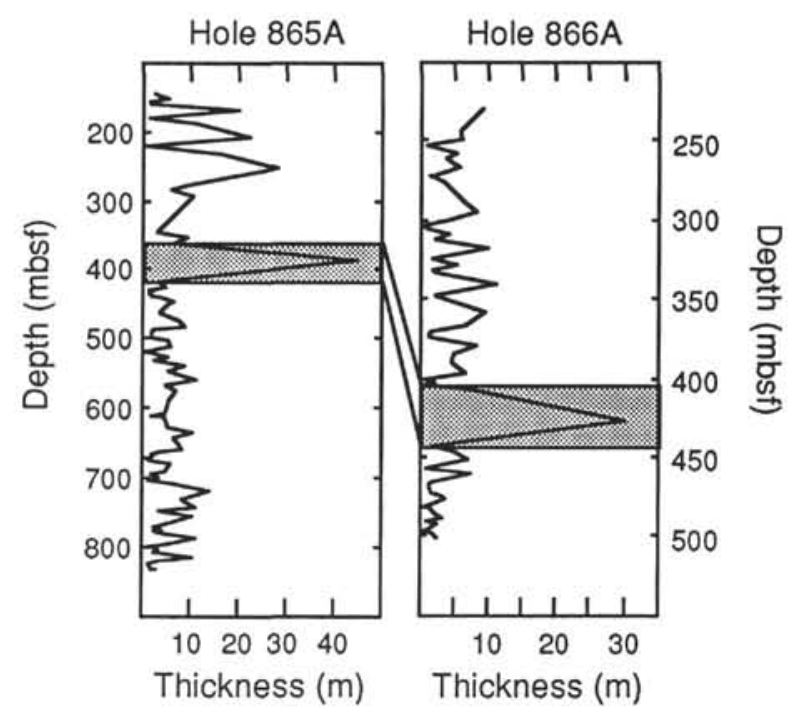

Figure 11. Comparison of upward-fining sequence thicknesses within the Albian portion of Hole 866A (top of logged section to $500 \mathrm{mbsf}$ ) and Hole $865 \mathrm{~A}$, which is entirely Albian in age. 